\title{
Complexos simpliciais finitos e o teorema de Euler
}

\author{
Marcelo Barbosa Viana
}

\author{
DisSERTAÇÃO APRESENTADA \\ AO \\ Instituto De Matemática e EstatísticA \\ DA \\ Universidade DE SÃo PAUlo \\ PARA \\ OBTENÇÃO DO TÍTULO \\ $\mathrm{DE}$ \\ Mestre em CiênCIAS
}

\author{
Programa: \\ Matemática \\ Orientador: Prof. Dr. Ivan Struchiner
}

Durante o desenvolvimento deste trabalho o autor recebeu auxílio financeiro da FAPEAM EDITAL No 001/2015 - RH - MESTRADO - V CHAMADA.

São Paulo, 09 de novembro de 2018 


\section{Complexos simpliciais finitos e o teorema de Euler}

Esta versão da dissertação contém as correções e alterações sugeridas pela Comissão Julgadora durante a defesa da versão original do trabalho, realizada em 09/11/2018. Uma cópia da versão original está disponível no Instituto de Matemática e Estatística da Universidade de São Paulo.

Comissão Julgadora:

- Prof. Dr. Ivan Struchiner (Orientador) - IME-USP

- Prof. Dr. Carlos Grossi - ICMC-USP

- Prof. Dr. Pedro Frejlich - UFRGS 


\section{Agradecimentos}

Ao findar mais esta etapa de minha vida acadêmica, os agradecimentos a todos aqueles que a tornaram possível são indispensáveis e aqui os faço com justiça e humildade.

Ao Senhor, nosso Deus, que no seu infinito e generoso amor, permitiu-nos a estadia na cidade de São Paulo e a convivência dos nossos mais novos amigos em segurança e permanente paz. Obrigado Senhor!

Ao meu Pai, Manoel "Mimi" dos Santos Viana (o primeiro Matemático que conheci!) e minha mãe, Marlene Barbosa Viana; Suas bençãos me fizeram uma pessoa melhor e pronta para o mundo, amo vocês. Minha esposa, Daiana "Dori" dos Santos Viana, minha companheira, minha amiga, meu amor e meu filho, Fágner "Doudou" Marcelo dos Santos Viana, agradeço por tudo. Esta é uma vitória em Família, amo vocês. Aos nossos padrinhos "4-ever", Ana Tereza e Paulo Wanderson Farias, obrigado amigos, obrigado por participarem da defesa deste trabalho.

Ao meu querido orientador Dr. Ivan Struchiner, um exemplo de profissional a ser seguido. Agradeço nossas conversas, discussões, etc, todas elas, sem exceção, serviram para o amadurecimento na busca do melhor pesquisar e trabalhar em Matemática. Sempre serei grato à sua gentileza e companheirismo e seguirei firme nos estudos da boa Matemática pois nossa parceria é, de agora, para sempre. Muito Obrigado.

Agradeço aos professores da querida Universidade Federal do Amazonas (UFAM) que sempre acreditaram no meu potencial para a coisa Matemática: Dr. Ivan Tribuzy (In Memoriam), Dr. Renato Tribuzy, Dr. Nilomar Vieira, Dr. Claudenir Rodrigues, Dr. Raul Rabelo, Dr. Roberto Cristovão, Dr. Roberto Prata, Dra. Flávia Morgana, Dr. Alfredo Wagner, Dr. Carlos Wagner, Dr. José Nazareno. Muito obrigado a todos.

Aos amigos do nosso grupo de estudo sob a tutela do prof. Ivan Struchiner. Esse grupo merece registro, faço-o aqui com todo orgulho: Gustavo Ignácio, meu irmão! Agradeço a amizade e parceria, todas valem ouro. Luciana Bonatto, a primeira de nós, sua gentileza e desenvoltura matemática nos enche de orgulho, satisfação em tê-la conhecido. Cristian Cárdenas e Genaro "Meu Bem" Chauca, nossos doutores. A dedicação de vocês mostraram que é possível obter sucesso dentro de uma área tão difícil da Matemática. Obrigado amigos e sucesso sempre.

A todos os colegas do mestrado/doutorado em Matemática do IME, secretaria/ MAT, biblioteca, reprografia e, em especial, aos professores do IME/USP: Dr. Raul Ortiz, Dr. Antônio de Pádua, Dra. Bárbara Valério e, destacando, o Prof. Dr. Odilon Luciano, pelas horas infinitas de sabedoria matemática e descontração, obrigado sempre.

Aos amigos do Instituto Federal do Acre (IFAC /Cruzeiro do Sul) que, através da compreensão e respeito, permitiram a conclusão deste trabalho: Orleinilson Batista, Narciso Melo, José Júlio César, Cristiano Ferreira, Jailson Freitas, Pedro Fabrício, Maiane Araújo, Marcondes Nicácio, Carpegiani Costa, Suelen Teles, Mara Rykelma, Ana Cláudia Garcia, Liliana Rodrigues, Lilliane Mar- 
tins (Dirge), Rodrigo Marciente (Diren), Ronegildo Silva (Cotep), Tamires Costa (Cogep), Kelvyla Silva, Nelzira Guedes (NAES), Israel Souza (Copie), meus agradecimentos.

Meus amigos de infância que sempre me apoiaram: Ivan Wallace, Rone Mário, Márcio Macedo, Paulo Wanderson, Fágner "Boby" Oliveira da Costa (In memoriam), os amigos e colegas de Porto Trombetas - PA, cujo convívio permitiu sonhar alto, e aprender mais e mais da matemática.

Obrigado especial aos meus primeiros professores da disciplina: Reginaldo "Fera" Távora, Paulo "Le Grand" Moraes, Nilza, Maria das Graças Távora, Juliana, enfim... Todos os professores da Escola Professor Jonathas Pontes Athias, seu apoio, suas dicas e verdades sobre o mundo fora Trombetas foram de suma importância para estes resultados alcançados e os que virão.

Obrigado queridos!!!

Ps.: Agradecimento especial para o senhor Nelson, a senhora Aparecida "Cida" e sua filha Rosângela por nos receber em São Paulo para a defesa deste importante trabalho. Obrigado.

Este trabalho teve apoio financeiro da Fundação de Amparo à Pesquisa do Estado do Amazonas - FAPEAM, que na pessoa da senhora Maria Iara Marialva Elisiário, recebe meus sinceros agradecimentos. 


\section{Dedicatória}

Aos professores Elon Lages Lima (In memoriam) e

Ivan de Azevedo Tribuzy (In memoriam), que na busca pela excelência Matemática expandiram as pesquisas nesta área aos quatro quantos deste enorme país. 
"Meu filho, você vai morar sozinho, portanto, Procure falar bem, vestir-se bem, ..., 'andar limpo' Trate bem as pessoas, sorria sempre ... Seja verdadeiro! Faça tudo isso e elas o receberão de braços abertos". "Mimi Viana" (Teu velho pai) 


\section{Resumo}

VIANA, M. B. Complexos simpliciais finitos e o teorema de Euler. 2018. Dissertação Instituto de Matemática e Estatística, Universidade de São Paulo, São Paulo, 2018.

Neste trabalho iremos apresentar uma releitura de um resultado clássico da topologia, na visão da topologia algébrica e em sua notação atual. A demonstração deste, apresentada por Cauchy (1813), é comentada de maneira crítica em Lima (1985a) e para esta apresentação destacaremos as definições, teoremas e entes básicos para o seu entendimento.

Palavras-chave: poliedro, homologia, topologia, Euler, Cauchy, simplexos. 


\section{Abstract}

VIANA, M. B. Finite simplicial complexes and the Euler theorem. 2018. Dissertação Instituto de Matemática e Estatística, Universidade de São Paulo, São Paulo, 2018.

In this work we will present a rereading of a classic topology result, in the view of the algebraic topology in its current notation. The proof of this, presented by Cauchy (1813), is critically commented on Lima (1985a) for which we will present the definitions, theorems, basic entities for their understanding.

Keywords: polyhedron, homology, topology, Euler, Cauchy, simplices . 


\section{Conteúdo}

Lista de Figuras $\quad$ XV

1 Introdução $\quad \mathbf{1}$

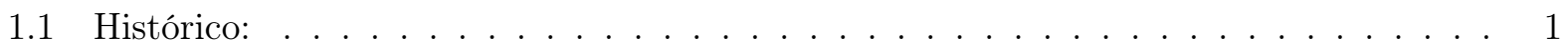

1.2 Organização: . . . . . . . . . . . . . . . . . . . . 2

2 Elementos de Topologia Algébrica $\quad 3$

2.1 Complexo Simplicial . . . . . . . . . . . . . . . . . . . . . . . . . . . 3

2.1 .1 Simplexos: . . . . . . . . . . . . . . . . . . . . . . 3

2.1 .2 Estruturas Simpliciais: . . . . . . . . . . . . . . . . . . . . 5

2.1 .3 Complexo Simplicial: . . . . . . . . . . . . . . . . . 6

2.1.4 Triângulação de um Espaço Topológico: . . . . . . . . . . . . . . . . . . 7

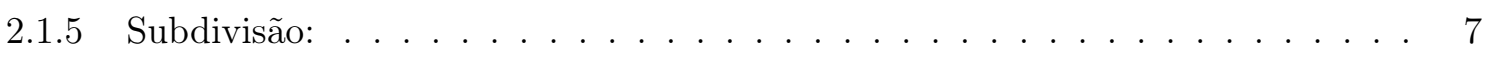

2.1.6 Número de Euler de um Complexo Simplicial Finito . . . . . . . . . . . . . . 10

2.2 Homologia Simplicial . . . . . . . . . . . . . . . . . . . . 11

2.2 .1 Orientação . . . . . . . . . . . . . . . . . . . . . . . . . 11

2.2 .2 Grupo das $p$-cadeias Simpliciais: . . . . . . . . . . . . . . . . 11

2.2 .3 Complexo de Cadeias Simpliciais: . . . . . . . . . . . . . . . . . . . . . . 12

2.2.4 Grupos de Homologia Simplicial: . . . . . . . . . . . . . . . . . . . 12

2.2 .5 Aplicações de Cadeias: . . . . . . . . . . . . . . . . . . . . . . . . . . 19

2.3 Pseudo - Variedades . . . . . . . . . . . . . . . . . . . . 21

2.3 .1 Orientação: . . . . . . . . . . . . . . . . . . . . . . . . . . 21

2.3 .2 Classe Fundamental: . . . . . . . . . . . . . . . . . . . . 22

2.3.3 Pseudo - Variedades com Bordo: . . . . . . . . . . . . . . . . . . . 22

2.4 Homologia Singular . . . . . . . . . . . . . . . . . . . . 23

2.4.1 Homologia Singular de $X$ com coeficientes em um anel $R$ : . . . . . . . . 23

2.4.2 Homotopia e Equivalência Homotópica: . . . . . . . . . . . . . . . 25

2.4 .3 Sequências Exatas . . . . . . . . . . . . . . . . . . . . . 28

2.4 .4 Sequência Longa do Par . . . . . . . . . . . . . . . . . . . . . . . . . . . . . . . . . . . . . . . . . . . . . . . . .

2.4 .5 Bom Par . . . . . . . . . . . . . . . . . . . . 34

2.5 Isomorfismo entre Homologias Simplicial e Singular . . . . . . . . . . . . . . . 35

2.5.1 Homologia Simplicial vs. Homologia Singular . . . . . . . . . . . . . . . 35

2.6 Aplicações . . . . . . . . . . . . . . . . . . . . . . 36

2.6.1 Homologia de $\mathbb{S}^{2}$ - via homologia simplicial: . . . . . . . . . . . . . 37

2.6.2 Homologia de $\mathbb{S}^{2}$ - via Bom Par . . . . . . . . . . . . . 38 
2.6.3 Homologia de $\mathbb{S}^{2}$ - via Mayer - Vietoris . . . . . . . . . . . . . . . 38

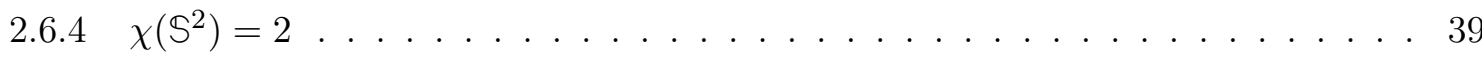

3 Teorema de Euler (Cauchy - Lima) $\quad 41$

3.1 Comentários Iniciais: . . . . . . . . . . . . . . . . . . . . . . . . . . 41

3.1.1 Teorema de Euler para Poliedros (Cauchy - 1813) . . . . . . . . . . . . . . 41

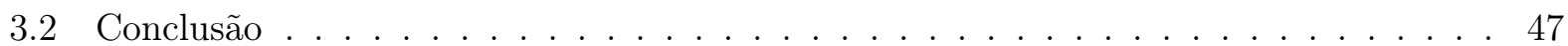

$\begin{array}{ll}\text { Bibliografia } & 49\end{array}$ 


\section{Lista de Figuras}

1.1 Poliedros: (a) convexo (b) não-convexo . . . . . . . . . . . . . . . . . . . . 1

$2.1 p$-simplexo padrão para $p=0,1,2$ e $3 \ldots \ldots \ldots \ldots \ldots$

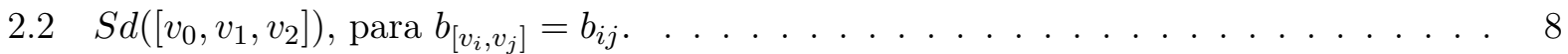

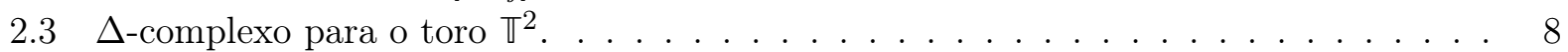

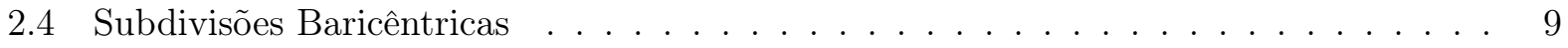

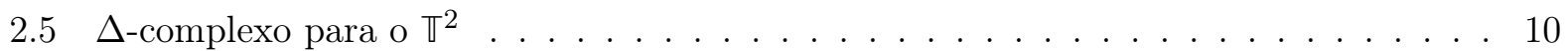

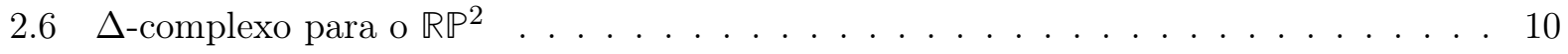

$2.7 \quad(a)$ e $(d)$ são árvores; $(b),(c)$ grafos, Kinsey (1993), pg. 92 . . . . . . . . . . . . . 10

2.8 Orientação de um simplexo. (Munkres $(1984)$ ) . . . . . . . . . . . . . . . 11

2.9 Bordo para um 2 e 3 -simplexo . . . . . . . . . . . . . . . . . . . . . . 12

2.10 Complexo Simplicial $K_{1}\left(\operatorname{dim} K_{1}=1\right)$ (Gallier e Quaintance $\left.(2016)\right) \ldots \ldots \ldots$

2.11 Complexo Simplicial $K_{2}\left(\operatorname{dim} K_{2}=2\right)$ (Gallier e Quaintance (2016)). . . . . . . . . . 14

2.12 Complexo Simplicial $K_{3}\left(\operatorname{dim}_{3}=2\right)$ (Gallier e Quaintance (2016)). . . . . . . . . . 15

2.13 Complexo Simplicial $K_{4}\left(\operatorname{dim} K_{4}=3\right)$ (Gallier e Quaintance (2016)) . . . . . . . . . . 17

$2.14 \Delta$-simplexo para o toro $\mathbb{T}^{2}$, $($ Hatcher $(2001)) \ldots \ldots \ldots \ldots \ldots \ldots$

2.15 Pseudo-variedades. . . . . . . . . . . . . . . . . . . . . 21

2.16 Faixa de Möbius como pseudo-variedade. . . . . . . . . . . . . . . . . . . 22

2.17 Espaços Homotopicamente Equivalentes (Hatcher (2001)) . . . . . . . . . . . . . . 27

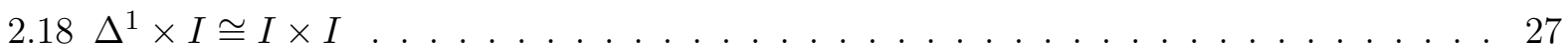

2.19 Triângulação da Esfera $\mathbb{S}^{2} \ldots \ldots \ldots$. . . . . . . . . . . . . . . . . 37

2.20 hipóteses em Mayer - Vietoris ． . . . . . . . . . . . . . . . . . . . . 39

2.21 Triangulações da Esfera $\mathbb{S}^{2}$.Brasselet e Thuy (2016) . . . . . . . . . . . . . . . . . . 40

3.1 Poliedros para os quais o teorema é válido. . . . . . . . . . . . . . . . . . . . 41

3.2 Poliedros para os quais o teorema não é válido. . . . . . . . . . . . . . . . . . 41

3.3 Ciclo que não é bordo em $\mathbb{t}^{2} \ldots \ldots \ldots \ldots \ldots \ldots$. . . . . . . . . . . . . . . 42

3.4 Pseudo-variedade de dimensão $2 \ldots \ldots \ldots \ldots$. . . . . . . . . . . . . . 42

3.5 Retirada de um 2-simplexo $\sigma_{k}$ da pseudo-variedade $K$. (Brasselet e Thuy (2016)) . . 43

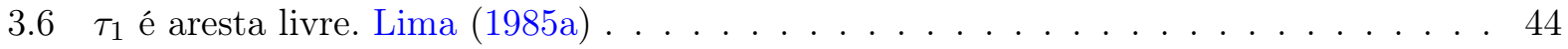

$3.7 \tau_{1}$ e $\tau_{2}$ são arestas livres. Lima $(1985 \mathrm{a}) \ldots \ldots \ldots$. . . . . . . . . . . . . . 45

$3.8 \tau_{1}$ e $\tau_{2}$ são arestas livres, mas nenhum $v$ o é. Lima (1985a) . . . . . . . . . . . . . . 45

$3.9 \tau_{1}, \tau_{2}, \tau_{3}$ são arestas livres, com nenhum vértice livre. Lima (1985a) . . . . . . . . . . 46

$3.10 \tau_{1}, \tau_{2}, \tau_{3}$ são arestas livres é $v_{0}$ vértice livre. Lima $(1985 \mathrm{a}) \ldots \ldots$. . . . . . . . . 46

$3.11 \tau_{1}, \tau_{2}, \tau_{3}$ são arestas livres e $v_{0}, v_{1}$ são vértices livres. Lima (1985a) . . . . . . . . . 47 


\section{Capítulo 1}

\section{Introdução}

\subsection{Histórico:}

Os estudos iniciais de Topologia Algébrica, que acreditamos ser tarefa para todo e qualquer estudante em Matemática, nos permitem olhar para resultados clássicos com visão crítica e reflexiva, dentre eles, destacamos os que envolvem poliedros (complexos simpliciais finitos) objetos de nosso estudo aqui apresentado.

Poliedros (suas propriedades geométricas, etc) já eram conhecidos pelos matemáticos gregos ${ }^{1}$ : Pitágoras, Euclides, entre outros. Com J. Kepler (1571-1630) chegaram a ser utilizados para o seu modelo inicial de sistema solar (Richeson (2012)) entretanto, as primeiras observações lógicas, foram feitas pelo Matemático e filósofo René Descartes que, juntamente com os demais, serviram ao ensino de geometria nas escolas da época.

Em 14 de novembro de 1750, em carta encaminhada a seu amigo Cristian Goldbach (1690 1764), Leonhard Euler (1707 - 1783) escreveu acerca de determinada observação a respeito dos poliedros e no ano seguinte apresentou uma prova. Esta observação é a que tempos depois ficaria conhecida como Fórmula de Euler para Poliedros.

Um poliedro é um objeto tridimensional composto por faces poligonais unidas uma as outras ao longo de segmentos de retas, chamados arestas e, estas interceptam-se em em um ponto, chamado vértice.
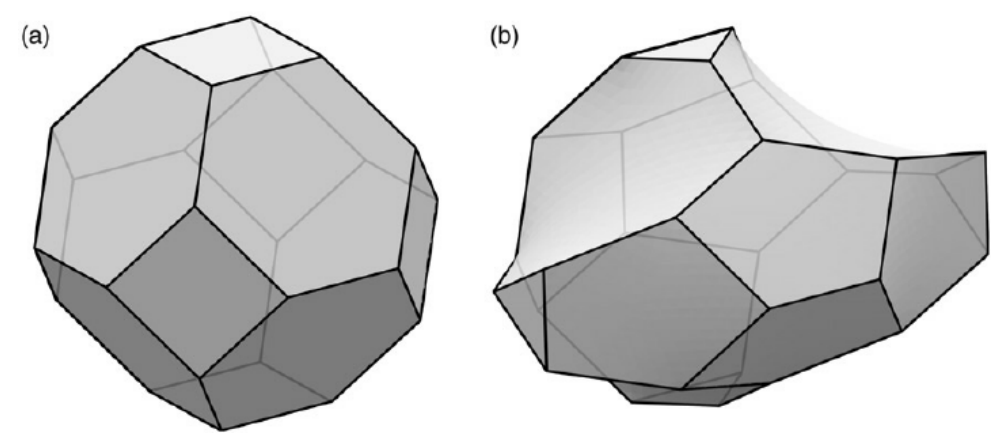

Figura 1.1: Poliedros: (a) convexo (b) não-convexo

Euler observou que para poliedros regulares, o número de vértices, arestas e faces $(V, A \text { e } F)^{2}$ satisfazem à relação:

$$
V-A+F=2 .
$$

O presente trabalho é uma releitura deste clássico resultado em matemática (o Teorema de Euler para Poliedros) e, para tanto, seguimos a exposição de Lima (1985a) ao assunto, onde apresenta,

\footnotetext{
${ }^{1}$ Uma bela introdução ao tema pode ser vista em Richeson (2012)

${ }^{2}$ Notação que não era utilizada pelo mesmo, (Richeson (2012))
} 
comenta e enriquece a demonstração feita por Cauchy (1789 -1857).

A blibliografia inclui diversas publicações sobre o tema e nossa abordagem destaca a importância da teoria de homologia para um melhor entendimento do assunto.

A linguagem atual da topologia algébrica será utilizada a fim de demonstrar a importância do teorema de Euler, bem com a própria característica de Euler que permite associar a um determinado complexo simplicial finito um invariante topológico.

\subsection{Organização:}

O presente trabalho se divide em duas partes: O capítulo (2) trata dos conceitos e resultados básicos da Topologia Algébrica e o conceito de Homologia (em especial a) Simplicial com ilustrações, exemplos de como calcular os grupos desta homologia, é objetivo deste capítulo. A definição de Complexo Simplicial (definição 2.1.7) e suas estruturas, em especial, os simplexos (definição 2.1.1) dão início a esta tarefa.

O conceito de triangulação (2.1.10) (quando existir) deste espaço é apresentado em seguida e a partir desse conceito calculamos grupos de homologia simplicial. A escolha da Homologia Simplicial (definição 2.2.9) não se deu por acaso. A apresentação geométrica e combinatória deste conceito exemplifica os resultados obtidos através de várias figuras ao longo do capítulo, onde destacamos os exemplos devidos a Gallier e Quaintance (2016).

O conceito de pseudo-variedade (definição 2.3.1) é apresentado neste capítulo e esta definição será importante ao resultado final deste trabalho. O principal conceito a ser apresentado nesta parte é o número de Euler (definição 2.1.16). Finalizaremos o capítulo definindo a homologia singular (definição 2.4.4) e mostraremos que os grupos de homologia singular são isomorfos aos de homologia simplicial o que permite a livre escolha destas ao se calcular estes grupos.

Já no terceiro capítulo (3) e com base na leitura do artigo principal à dissertação (Lima (1985a)), trataremos do teorema de Euler e verificaremos que a demonstração formulada por Cauchy (que é geométrica) de fato não demonstra o resultado de Euler para poliedros homeomorfos a esfera (pois utiliza técnicas avançadas da topologia álgebrica). Apresentaremos um enunciado alternativo ao de Cauchy e faremos a demonstração deste resultado geométrico.

As referências ao trabalho são apresentadas ao final do texto (3.2). 


\section{Capítulo 2}

\section{Elementos de Topologia Algébrica}

Neste capítulo listaremos as principais definições e resultados necessários a esta dissertação, onde destacamos, inicialmente, os complexos simpliciais e os grupos de homologia simplicial.

\subsection{Complexo Simplicial}

Definiremos os grupos de homologia simplicial e, para isto, discutiremos a classe de espaços para os quais estes são definidos. Esta é a classe dos poliedros. Um poliedro é um espaço cuja construção pode ser feita a partir da união de blocos formados por: pontos, segmentos de retas, triângulos, tetraedros e seus análagos em dimensões maiores. Esta união é feita "colando" estes blocos ao longo de suas "faces" comuns. Discutiremos este tipo de construção introduzindo: notações, definições e resultados necessários a este entendimento.

Historicamente, homologia simplicial surgiu no início da década de 1920, mais de trinta anos após a publicação do primeiro artigo de Poincaré em 1892 e até a década de 1930, os grupos de homologia não eram definidos. Neste período, os matemáticos trabalhavam com invariantes numéricos tais como: números de Betti (1823 - 1892) e torções, noções atuais na época e foi neste período que Emmy Noether (1882 - 1935) fez uma importante contribuição com a introdução dos conceitos de grupos (entes algébricos) ao estudo de homologia, que nesta seção, serão nossos objetos de estudo.

O caráter geométrico e combinatório das estruturas simpliciais nos permitem uma melhor compreensão dos conceitos a serem tratados, dentre os quais destacamos o cálculo dos grupos de homologia simplicial de um determinado espaço topológico.

\subsubsection{Simplexos:}

A principal estrutura de que trataremos aqui é o simplexo. Estes entes geométricos/combinatórios, oriundos da definição de espaço afim ${ }^{1}$, são importantes ao objeto de estudo a que esta dissertação se propõe.

Definição 2.1.1 Diremos que $(p+1)$ pontos $v_{0}, \ldots, v_{p}$ em $\mathbb{R}^{n}$ são independentes (em posição genérica) se o conjunto $\left\{v_{1}-v_{0}, \ldots, v_{p}-v_{0}\right\}$ é linearmente independente ${ }^{2}$. Um ponto $x \in \mathbb{R}^{n}$ será linearmente dependente dos pontos $v_{0}, \ldots, v_{p}$ se o vetor $x-v_{0}$ for dependente dos vetores $v_{i}-v_{0}$ para $i=1, \ldots, p$.

Exemplo 2.1.1 Em consequência da definição, dois pontos distintos, três pontos não colineares e quatro pontos que não pertençam ao mesmo plano (isto é, não coplanares) são independentes.

Definição 2.1.2 Um ponto $x \in \mathbb{R}^{n}$ será uma combinação afim de pontos $v_{0}, v_{1}, \ldots, v_{p} \in \mathbb{R}^{n}$ quando, $x=\sum_{i=0}^{p} \lambda_{i} v_{i}$ para $\sum \lambda_{i}=1$. Se, além disso, tivermos os $\lambda_{i} \geq 0, \forall i=0, \ldots, p$ então $x$

\footnotetext{
${ }^{1} \mathrm{O}$ caráter geométrico/combinatório dos espaços afins pode ser visto em Rotman (1988), capítulo 2, pag. 31.

${ }^{2}$ Isto, no sentido de Álgebra Linear (Hoffman e Kunze (1971)).
} 
será uma combinação convexa dos $v_{i}$. Um conjunto $X \subset \mathbb{R}^{n}$ é convexo se, e somente se, toda combinação convexa de elementos de $X$ pertence ao próprio $X$.

Com as definições anteriores podemos, de maneira geral, caracterizar a independência pontual em $\mathbb{R}^{n}$ através do seguinte resultado: (Lima (2012))

Proposição 2.1.1 Dados $v_{0}, v_{1}, \ldots, v_{p}$ pontos de $\mathbb{R}^{n}$, as seguintes afirmações são equivalentes:

(1) $v_{0}, v_{1}, \ldots, v_{p}$ são pontos independentes;

(2) Se as combinações afins $x=\sum_{i=0}^{p} \lambda_{i} v_{i}$ e $y=\sum_{i=0}^{p} \lambda_{i}^{\prime} v_{i}$ são iguais, então $\lambda_{0}=\lambda_{0}^{\prime}, \lambda_{1}=$ $\lambda_{1}^{\prime}, \ldots, \lambda_{p}=\lambda_{p}^{\prime}$.

Demonstração 2.1.1.1 Supondo a validade de (1) e que se tenha $x=y$, podemos escrever, por hipótese, para $\sum_{i=0}^{p} \lambda_{i}=1, \lambda_{0}=1-\left(\lambda_{1}+\ldots+\lambda_{p}\right)=1-\sum_{i=1}^{p} \lambda_{i}$. Como $x=\sum_{i=0}^{p} \lambda_{i} v_{i}$, segue que:

$$
\begin{aligned}
x & =\lambda_{0} v_{0}+\sum_{i=1}^{p} \lambda_{i} v_{i} \\
x & =\left(1-\sum_{i=1}^{p} \lambda_{i}\right) v_{0}+\sum_{i=1}^{p} \lambda_{i} v_{i} \\
x & =v_{0}+\sum_{i=1}^{p} \lambda_{i}\left(v_{i}-v_{0}\right)
\end{aligned}
$$

e analogamente,

$$
y=v_{0}+\sum_{i=1}^{p} \lambda_{i}^{\prime}\left(v_{i}-v_{0}\right) .
$$

Por hipótese

$$
\not \gamma+\sum_{i=1}^{p} \lambda_{i}\left(v_{i}-v_{0}\right)=y_{0}+\sum_{i=1}^{p} \lambda_{i}^{\prime}\left(v_{i}-v_{0}\right)
$$

Então,

$$
\sum_{i=1}^{p}\left(\lambda_{i}-\lambda_{i}^{\prime}\right)\left(v_{i}-v_{0}\right)=0 \in \mathbb{R}^{n} .
$$

Pela independência linear de $v_{i}-v_{0}$ segue que $\lambda_{i}=\lambda_{i}^{\prime} \forall i=1, \ldots, p$, desta forma $\lambda_{0}=\lambda_{0}^{\prime}$ e assim, (1) $\Rightarrow(2)$.

Reciprocamente, se a afirmação (2) é válida e afirmarmos, por absurdo, que algum dos $v_{i}-v_{0}$, por exemplo $v_{1}-v_{0}$, se escreve como combinação dos demais, teremos:

$$
v_{1}-v_{0}=\sum_{i=2}^{p} \lambda_{i}\left(v_{i}-v_{0}\right) .
$$

E portanto

$$
1 v_{1}=\left(1-\lambda_{2}-\lambda_{3}-\ldots-\lambda_{p}\right) v_{0}+0 v_{1}+\lambda_{2} v_{2}+\lambda_{3} v_{3}+\ldots+\lambda_{p} v_{p} .
$$

Teremos assim, formas distintas de escrever pontos iguais em $\mathbb{R}^{n}$ o que contraria (2). Portanto, todos os $v_{i}-v_{0}$ são linearmente independentes e pela definição 2.1.1 vale (1).

Logo $(2) \Rightarrow(1)$

Garantida a independência de pontos em $\mathbb{R}^{n}$ apresentamos uma classe especial de objetos do espaço. 
Definição 2.1.3 Um p-simplexo $\sigma$ em $\mathbb{R}^{n}$ é o conjunto (fecho convexo) de todas as combinações convexas dos $(p+1)$ pontos $v_{i} \in \mathbb{R}^{n}$, independentes, com representação $\sigma=\left(v_{0}, \ldots, v_{p}\right)$ onde cada $v_{i}$ é chamado vértice sendo $i=0, \ldots, p$.

Observação 2.1.1 Se $x \in\left(v_{0}, \ldots, v_{p}\right)$, então $x=\sum_{i=0}^{p} \lambda_{i} v_{i}, 0 \leq \lambda_{i} \leq 1 e \sum_{i=0}^{p} \lambda_{i}=1$, com o auxílio da proposição 2.1.1. Os números $\lambda_{i}$ são chamados coordenadas baricêntricas de $x$ em relação aos vértices $v_{0}, \ldots, v_{p}$.

Um exemplo importante e que será de grande valia para nosso trabalho é o simplexo padrão:

Definição 2.1.4 Um p-simplexo padrão $\Delta^{p}$ é o simplexo em $\mathbb{R}^{n}$ cujos vértices são exatamente os vetores $e_{i}$ da base canônica do espaço $\mathbb{R}^{n}$, isto é,

$$
\Delta^{p}=\left\{x=\sum_{i=0}^{p} \lambda_{i} e_{i} \mid \sum_{i=0}^{p} \lambda_{i}=1,0 \leq \lambda_{i} \leq 1, e_{i}=(0, \ldots, \underbrace{1}_{i}, \ldots, 0)\right\} .
$$
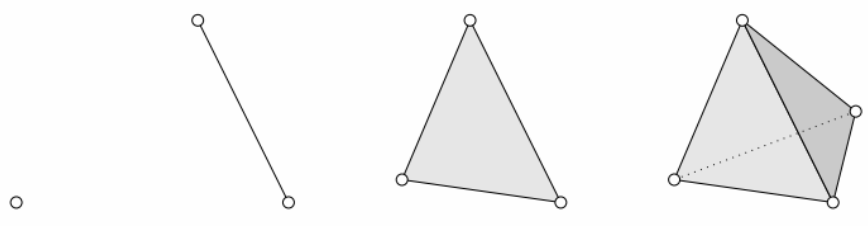

Figura 2.1: $p$-simplexo padrão para $p=0,1,2$ e 3

\subsubsection{Estruturas Simpliciais:}

Com a introdução anterior da definição de simplexo, passaremos a destacar em seguida os seus elementos estruturais.

Definição 2.1.5 Seja $\sigma$ um p-simplexo em $\mathbb{R}^{n}$. Uma face de $\sigma$ é um simplexo $\tau$ tal que, todo vértice de $\tau$ é, também vértice de $\sigma$. Usaremos $\tau \leq \sigma$ quando $\tau$ for face de $\sigma$ e no caso de $\tau \leq \sigma$ $\operatorname{com} \tau \neq \sigma$, diremos que $\tau$ é face própria de $\sigma$.

Observação 2.1.2 Qualquer simplexo gerado por algum subconjunto de $\left\{v_{0}, \ldots, v_{p}\right\}$ será uma face deste, em especial, a face $\left(v_{0}, \ldots, v_{i-1}, v_{i+1}, \ldots, v_{p}\right)$ é chamada a face oposta ao vértice $v_{i}$.

Definição 2.1.6 Dado um simplexo $\sigma$, definimos o bordo de $\sigma$ como sendo $\partial \sigma=\{\tau \leq \sigma \mid \tau \neq \sigma\}$, $o$ conjunto formado pelas faces próprias de $\sigma$. O interior de $\sigma$ será o conjunto $\sigma=\sigma-\partial \sigma$, também conhecido como simplexo aberto.

Observação 2.1.3 Um ponto $x \in \sigma$ com coordenadas baricêntricas $\lambda_{i}$ pertence $\grave{a} \stackrel{\circ}{\sigma} \Longleftrightarrow \lambda_{i}>0$, $\forall i$. Um ponto $x \in \sigma$ pertence à $\partial \sigma \Longleftrightarrow \lambda_{i}=0$ para algum $i$.

Proposição 2.1.2 Se $x \in \sigma$, tomando $\left\{\lambda_{i}(x)\right\}$ o conjunto formado pelas coordenadas baricêntricas de $x$ na observação 2.1.1, podemos listar algumas propriedades básicas dos simplexos. (Munkres (1984), pp. 4 e 5 .)

(1) As coordenadas baricêntricas $\lambda_{i}$ de $x$ com respeito a $v_{0}, \ldots, v_{p}$ são funções contínuas de $x$.

(2) $\sigma=\left(v_{0}, \ldots, v_{p}\right)$ é a união de todos os segmentos de retas que ligam o vértice $v_{0}$ a pontos do simplexo $\left(v_{1}, \ldots, v_{p}\right)$. Dois segmentos quaisquer desta união se interceptam apenas em $v_{0}$. 
(3) $\sigma$ é conjunto convexo e compacto em $\mathbb{R}^{n}$.

(4) Dado $\sigma \subset \mathbb{R}^{n}$, existe apenas um conjunto de pontos independentes em $\mathbb{R}^{n}$ que geram $\sigma$.

(5) Existe um homeomorfismo entre $\sigma$ e a bola unitária $B^{n}=\left\{x \in \mathbb{R}^{n}:\|x\| \leq 1\right\} \subset \mathbb{R}^{n}$ que leva $\partial \sigma$ à esfera unitária $S^{n-1}$.

Este item (5) é de extrema importância ao nosso estudo.

\subsubsection{Complexo Simplicial:}

Unindo as estruturas simpliciais, chegamos assim, ao complexo simplicial.

Definição 2.1.7 Um complexo simplicial finito $K$ (ou Poliedro) é um conjunto finito de simplexos em $\mathbb{R}^{n}$ no qual:

(1) Se $\sigma \in K$ e $\tau \leq \sigma \Rightarrow \tau \in K$,

(2) Se $\sigma, \tau \in K \Rightarrow \sigma \cap \tau=\emptyset$ ou $\sigma \cap \tau \leq \sigma, \tau$ (face comum),

(3) Todo $\sigma \in K$ é face de um número finito de simplexos de $K$.

Exemplo 2.1.2 O poliedro mais simples é o próprio p-simplexo com suas faces. Variando $p=$ $0,1,2,3$, teremos, respectivamente, o ponto, um segmento de reta, um triângulo (cheio) e um tetraedro (sólido) (Lima (2012), pg. 85)

Definição 2.1.8 Um subcomplexo simplicial $L$ de $K$, com $K$, complexo simplicial finito, é um subconjunto $L \subset K$ tal que $L$ é, também, um complexo simplicial.

Como exemplo importante de subcomplexo simplicial destacaremos o $p$-esqueleto, conceito este que segue:

Exemplo 2.1.3 O p-esqueleto $K^{(p)}=\{\sigma \in K \mid \sigma$ é um q-simplexo, $q \leq p\}$ de $K$ é um subcomplexo simplicial, tal que $K^{(0)} \subset K^{(1)} \subset \ldots \subset K^{(p)} \subset \ldots$ onde $K^{(0)}$ é formado apenas pelos vértices de K. Dizemos que $K$ tem dimensão $p$ se $K^{(p-1)} \neq K$ e $K^{(p)}=K$.

Observação 2.1.4 Neste trabalho trataremos apenas de complexos simpliciais finitos e portanto, de dimensão finita, $p<\infty$. Nos casos de dimensão infinita, teremos $\operatorname{dim} K=\infty$ se $K^{(p)} \neq K, \forall p$ (Munkres (1984)).

Definição 2.1.9 O suporte (ou realização geométrica) de $K$ é o espaço topológico $|K|$, formado pela união de todos os simplexos $\sigma \in K$, isto é,

$$
|K|=\bigcup_{\sigma \in K} \sigma .
$$

Onde a topologia é dada por $A \subset|K|$ é aberto $\Longleftrightarrow A \cap \sigma$ é aberto, $\forall \sigma$.

Observação 2.1.5 Esta topologia coincide com a topologia induzida de $\mathbb{R}^{n}$ para complexos simpliciais finitos. 


\subsubsection{Triângulação de um Espaço Topológico:}

Definição 2.1.10 Seja $X$ um espaço topológico. Uma triangulação (ou estrutura simplicial) em $X$ (quando existir) é um poliedro $K$ e um homeomorfismo $t:|K| \rightarrow X$. Se existir triangulação, diremos que $X$ é triangulável. Uma triangulação (excetuando-se o caso $X=\emptyset$ ) não é única.

\section{Exemplo 2.1.4}

(1) Se $\sigma$ é um $p$-simplexo, então $K=\{\tau \mid \tau \leq \sigma\}$ é um complexo simplicial e $|K| \cong D^{n}$. Portanto $D^{n}$ é triangulável.

(2) $K^{\prime}=\{\tau \mid \tau \leq \sigma, \tau \neq \sigma\}=\partial \sigma$ é um complexo e $\left|K^{\prime}\right| \cong \mathbb{S}^{n-1}$ para $\sigma$ simplexo de $K$.

(3) A menor triangulação do toro $\mathbb{T}^{2}$ tem 14 triângulos (2-simplexos). (Massey (1967))

(4) Toda variedade suave é triangulável. (Munkres (1966))

\subsubsection{Subdivisão:}

Um caso especial de triangulação de um complexo simplicial e a subdivisão baricêntrica, cujas características nos permitem resultados mais gerais sobre o complexo com relações métricas agradáveis aos cálculos subsequentes.

Definição 2.1.11 O baricentro de um p-simplexo $\sigma$ é o ponto $b_{\sigma}$ cujas coordenadas baricêntricas são $\left(\frac{1}{p+1}, \ldots, \frac{1}{p+1}\right)$, isto é,

$$
b_{\sigma}=\left(\frac{1}{p+1}\right) \sum_{i=0}^{p} v_{i} .
$$

Observação 2.1.6 O ponto $b_{\sigma}$ está em posição geral em relação à $\partial \sigma$, isto é, dados pontos $x, y \in \partial \sigma$, os segmentos $\left[b_{\sigma}, x\right] e\left[b_{\sigma}, y\right]$ possuem apenas o ponto $b_{\sigma}$ em comum.

Exemplo 2.1.5 Alguns exemplos em dimensão baixa ilustram nossa definição.

(1) Se $\sigma_{0}=\left(v_{0}\right) \Rightarrow b_{\sigma_{0}}=v_{0}$.

(2) Se $\sigma_{1}=\left(v_{0}, v_{1}\right) \Rightarrow b_{\sigma_{1}}=\frac{1}{2}\left(v_{0}+v_{1}\right)$.

(3) Se $\sigma_{2}=\left(v_{0}, v_{1}, v_{2}\right) \Rightarrow b_{\sigma_{2}}=\frac{1}{3}\left(v_{0}+v_{1}+v_{2}\right)$.

Definição 2.1.12 (Subdivisão baricêntrica) Seja K um complexo simplicial. A (primeira) subdivisão baricêntrica de $K$ é uma subdivisão do complexo simplicial $K$, representada por

$$
S d(K)=\left\{\left(b_{\sigma_{0}}, \ldots, b_{\sigma_{k}}\right) \mid b_{\sigma_{i}} \text { é o baricentro de } \sigma_{i} \in K, \sigma_{0}<\sigma_{1}<\ldots<\sigma_{k}\right\}
$$

Veremos a seguir algumas propriedades da subdivisão baricêntrica.

Definição 2.1.13 O diâmetro de um p-simplexo $\sigma=\left(v_{0}, \ldots, v_{p}\right)$ é o número

$$
\operatorname{Diam}(\sigma)=\operatorname{máx}\left\{\left|v_{i}-v_{j}\right|\right\}, i, j=0,1, \ldots, p
$$

Podemos relacionar o tamanho dos simplexos gerados a partir dos simplexos originais do complexo simplicial inicial através do seguinte resultado:

Teorema 2.1.1 Seja $\sigma$ um p-simplexo tal que Diam $(\sigma)=d$. Se $\sigma^{\prime}$ é a sua primeira subdivisão baricêntrica então para $\tau \leq \sigma^{\prime}$ tem-se

$$
\operatorname{Diam}(\tau) \leq\left(\frac{p}{p+1}\right) d
$$



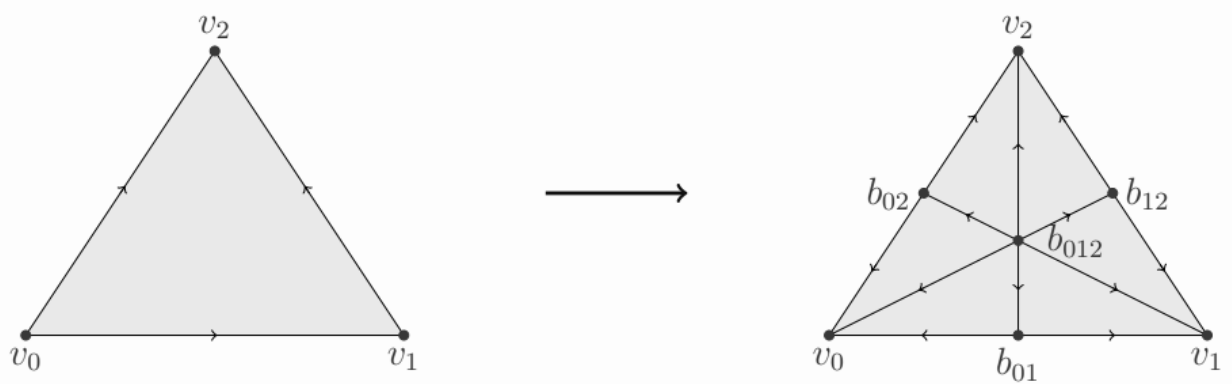

Figura 2.2: $S d\left(\left[v_{0}, v_{1}, v_{2}\right]\right)$, para $b_{\left[v_{i}, v_{j}\right]}=b_{i j}$.

Demonstração: Ver Lima (2012), pp. 106

A iteração do processo de subdivisão baricêntrica de um complexo simplicial permite-nos reduzir o tamanho dos novos simplexos com diâmetros cada vez menores. Vale portanto que:

Corolário 2.1.1.1 Sejam $K$ um complexo simplicial finito e um número real $\epsilon>0$. Existe $n \in \mathbb{N}$, tal que para todo $\tau \in K^{(n)}$ (n-ésima subdivisão baricêntrica) vale $\operatorname{Diam}(\tau)<\epsilon$.

Podemos triangular um espaço utilizando o conceito de estrutura de $\Delta$-complexo que apresentaremos a seguir.

Definição 2.1.14 Uma estrutura $\Delta$-complexo para um espaço topológico $X$ é uma coleção de simplexos $S=\left\{\sigma_{\alpha}: \Delta^{n} \rightarrow X \mid n=n(\alpha)\right\}$ tal que:

(1) $\bigcup_{\alpha} \sigma_{\alpha}=X$;

(2) $\left.\sigma_{\alpha}\right|_{\Delta^{n}}$ é injetor e $\forall x \in X, \exists$ ! $\alpha$ tal que $x \in \operatorname{Im}\left(\left.\sigma_{\alpha}\right|_{\Delta^{n}}\right)$;

(3) Se $\Delta_{i}^{n-1}=\left(e_{0}, \ldots, \hat{e}_{i}, \ldots, e_{n}\right)$ é uma face de $\Delta^{n}$ tem-se $\sigma_{\beta}=\left.\sigma_{\alpha}\right|_{\Delta^{n-1}} \in S$;

(4) $A \subset X$ é aberto $\Longleftrightarrow \sigma_{\alpha}^{-1}(A) \subset \Delta^{n}$ é aberto, $\forall \alpha$.
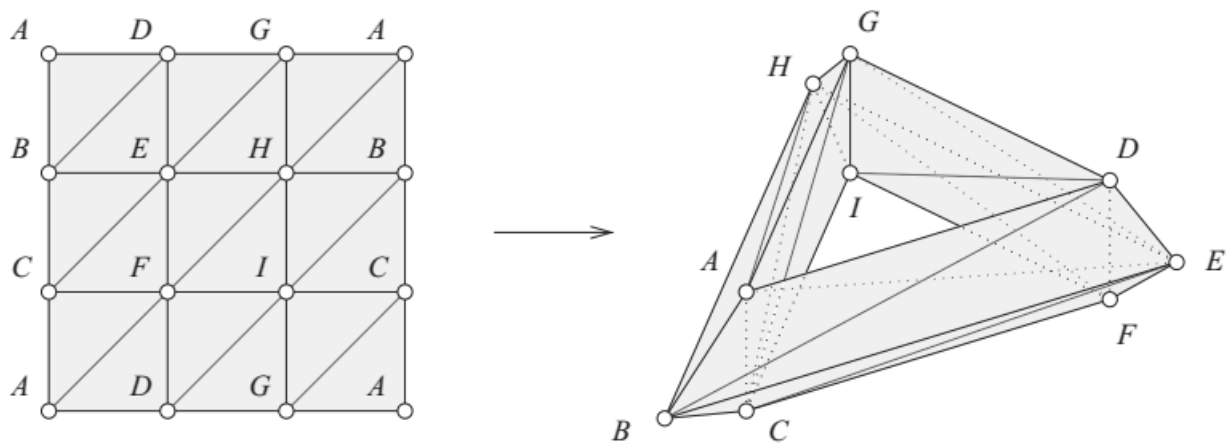

Figura 2.3: $\Delta$-complexo para o toro $\mathbb{T}^{2}$.

Definição 2.1.15 Sejam $K$ e $L$ complexos simpliciais finitos. Uma aplicação $f:|K| \rightarrow|L|$ é uma aplicação simplicial se:

(1) $\left(v_{\alpha_{1}}, \ldots, v_{\alpha_{l}}\right) \in K \Longrightarrow f\left(v_{\alpha_{1}}\right), \ldots, f\left(v_{\alpha_{l}}\right)$ são vértices de algum simplexo de $L$.

(2) $f$ é linear nos simplexos, isto é, $f\left(\sum \lambda_{i} v_{\alpha_{i}}\right)=\sum \lambda_{i} f\left(v_{\alpha_{i}}\right)$. 
Observação 2.1.7 $\left(f\left(v_{\alpha_{1}}\right), \ldots, f\left(v_{\alpha_{l}}\right)\right)$ não precisa ser um simplexo de L pois, $f\left(v_{\alpha_{1}}\right), \ldots, f\left(v_{\alpha_{l}}\right)$ não precisam ser distintos.

Proposição 2.1.3 Seja $X$ um espaço topológico. Uma estrutura $\Delta$-complexo para $X$ é uma triangulação deste espaço se, e somente se, valem:

(1) Cada p-simplexo de $X\left(\operatorname{Im}\left(\sigma_{\alpha}\right)\right)$ tem $(p+1)$ vértices distintos;

(2) Nenhum outro simplexo de $X$ tem o mesmo conjunto de vértices.

Demonstração 2.1.3.1 Seja $K$ uma estrutura $\Delta$-complexo para $X$. Se vale (1) para $K$, então toda aplicação característica $\sigma_{\alpha}: K \rightarrow X$ é bijetiva. Se vale, também, (2), dados $\sigma_{1}, \sigma_{2} \in K^{(1)}$ (divisão baricêntrica de K) tem-se: $\sigma_{1} \cap \sigma_{2}=\emptyset$ ou $\sigma_{1} \cap \sigma_{2} \leq \sigma_{1}, \sigma_{2}$ e neste caso $K$ é uma triangulação de $X$. De fato, dado $K^{(1)}=\left\langle b_{\sigma_{0}}, \ldots, b_{\sigma_{n}}\right\rangle$, com $\sigma_{0}<\sigma_{1}<\ldots<\sigma_{n} \in K$ toda aplicação simplicial leva baricentros em baricentros e desta forma podemos identificar $K$ com $K^{(1)}$ de modo natural, assim $\left|K^{(1)}\right| \approx|K|$. Agora se $\sigma_{0}<\sigma_{1}<\ldots<\sigma_{n} \in K$, então $b_{\sigma_{n}} \in \operatorname{Im}\left(\sigma_{n}\right)$, enquanto $b_{\sigma_{i}} \in \partial \sigma_{n}$ para todo $i=0, \ldots, n-1$. Portanto, $b_{\sigma_{n}} \neq b_{\sigma_{i}}$ para $i \neq n$. Continuando o processo por indução, teremos que todos os vértices de um simplexo em $K^{(1)}$ são diferentes de $\left|K^{(1)}\right|$.

Supondo $L \neq K$ outra estrutura $\Delta$-complexo nas condições anteriores e $\sigma$ um $n$-simplexo em $L$. Se $f_{\sigma}$ não for injetora, então algumas faces de $\sigma$ distintas serão identificadas. Neste caso, todas as aplicações características, e em particular $\sigma$, possuem no máximo n vértices contradizendo, portanto, a propriedade (1).

Desta forma, diferentes simplexos em $|L|$ podem ter mais de um lado comum e assim L não é necessariamente um complexo simplicial.

Uma subdivisão $K^{\prime}$ mantém a propriedade de um complexo simplicial: a interseção de dois simplexos em $\left|K^{\prime}\right|$ é um face comum (ou vazia).
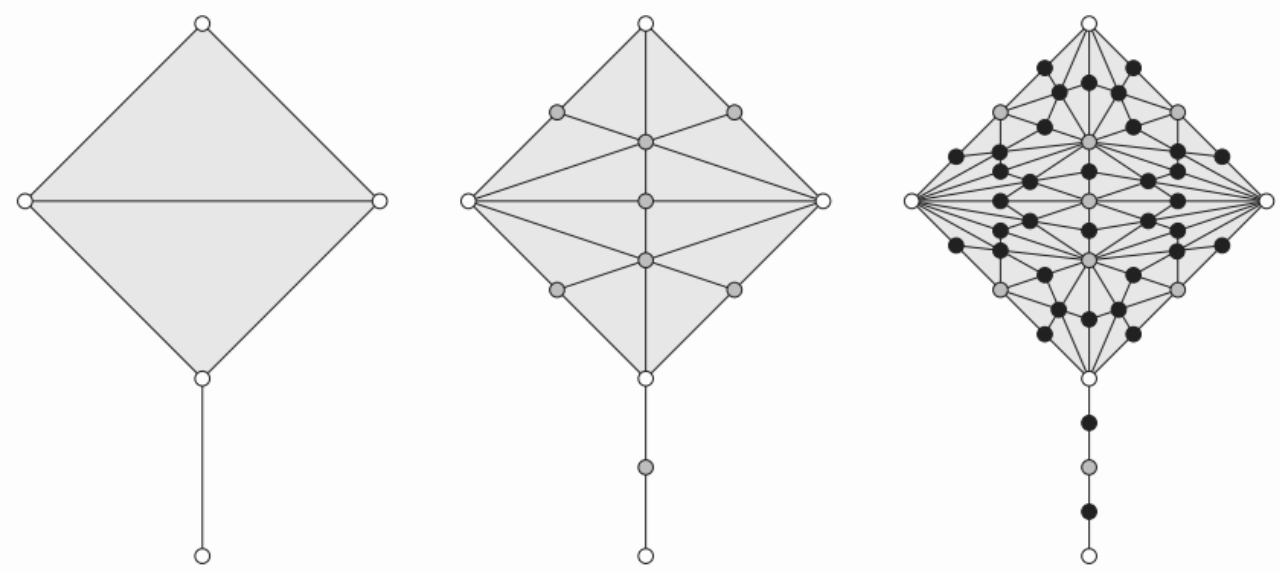

Figura 2.4: Subdivisões Baricêntricas

Exemplo 2.1.6 Uma estrutura de $\Delta$-complexo para o toro $\mathbb{T}^{2}$ é obtida a partir da união de dois 2-simplexos (figura 2.5) que dividem a representação (identificação) deste espaço (um retângulo com lados e vértices identificados) cortado ao longo da diagonal deste. 

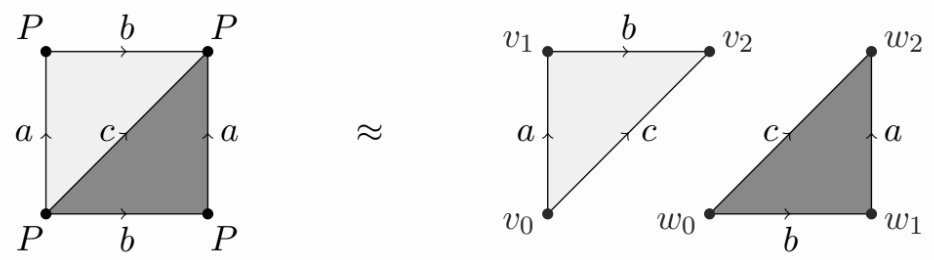

Figura 2.5: $\Delta$-complexo para o $\mathbb{T}^{2}$

Exemplo 2.1.7 De mesma forma, a partir da identificação do espaço projetivo $\mathbb{R P}^{2}$, obtemos uma estrutura de $\Delta$-complexo neste espaço:
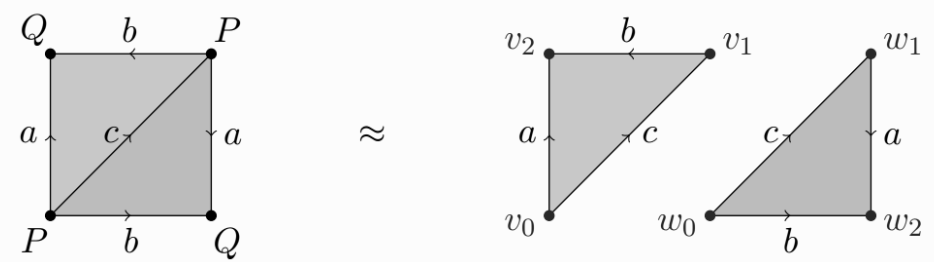

Figura 2.6: $\Delta$-complexo para o $\mathbb{R P}^{2}$

\subsubsection{Número de Euler de um Complexo Simplicial Finito}

Uma característica presente na triangulação (quando existe) de um espaço topológico $X$ é dada pela característica de Euler, número associado à quantidade dos simplexos (de dimensões diversas) que lá se encontram. (Brasselet e Thuy (2016), Lima (1984), Lima (1985b)).

Definição 2.1.16 Seja $K$ um complexo simplicial finito com dimK $=p$. A característica de Euler deste complexo é a soma alternada do número de $i$-simplexos em $K, 0 \leq i \leq p$, isto é,

$$
\chi(K)=\sum_{i=0}^{p}(-1)^{i} \#\left(\sigma_{i}\right) .
$$

Onde \# $\left(\sigma_{i}\right)$ é o número dos $i$-simplexos em $K$.

Exemplo 2.1.8 Dado um grafo $\Gamma$ (complexo simplicial conexo formado apenas por simplexos de dimensões: 0 e 1) tem-se $\chi(\Gamma)=\#\left(\sigma_{0}\right)-\#\left(\sigma_{1}\right)$.

Exemplo 2.1.9 Uma árvore $A$ é um grafo conexo sem ciclos (laços) e portanto $\chi(A)=1$. (vale a reciproca Kinsey (1993), pg. 93)

(a)

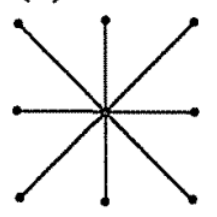

(b)

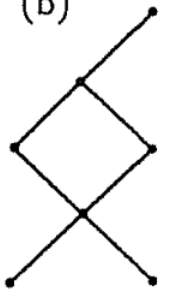

(c)

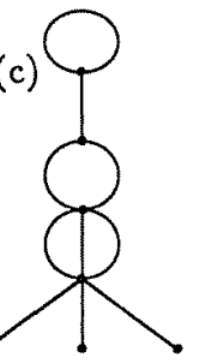

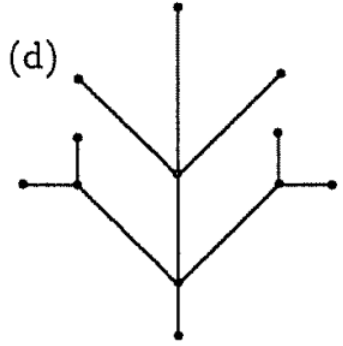

Figura 2.7: $(a)$ e $(d)$ são árvores; $(b),(c)$ grafos, Kinsey (1993), pg. 92. 


\subsection{Homologia Simplicial}

\subsubsection{Orientação:}

Definição 2.2.1 (Simplexos orientados) Um 0-simplexo orientado é um par $(\sigma, \epsilon)$ onde $\sigma e ́$ um 0 -simplexo $\sigma=(v)$ e $\epsilon= \pm 1$

Definição 2.2.2 Se $p>0$, uma orientação em $\sigma=\left(v_{0}, \ldots, v_{p}\right)$ é uma escolha de uma classe de equivalência na ordem dos vértices de $\sigma$, onde duas ordens são equivalentes se diferem por uma permutação par. Notação $\left[v_{0}, \ldots, v_{p}\right]=\left(v_{0}, \ldots, v_{p}\right)$ para $v_{0}<v_{1}<\ldots<v_{p}$.

Definição 2.2.3 (Orientação Induzida) $S e \sigma=\left[v_{0}, \ldots, v_{p}\right]$ dado $\tau \leq \sigma, \sigma$ induz uma orientação em $\tau=\left(v_{0}, \ldots, \widehat{v}_{i}, \ldots, v_{p}\right)$ (diferente da orientação de $\tau$ dada pela ordem dos vértices de $\sigma$ ) $\tau=(-1)^{i}\left[v_{0}, \ldots, \widehat{v}_{i}, \ldots, v_{p}\right]$.
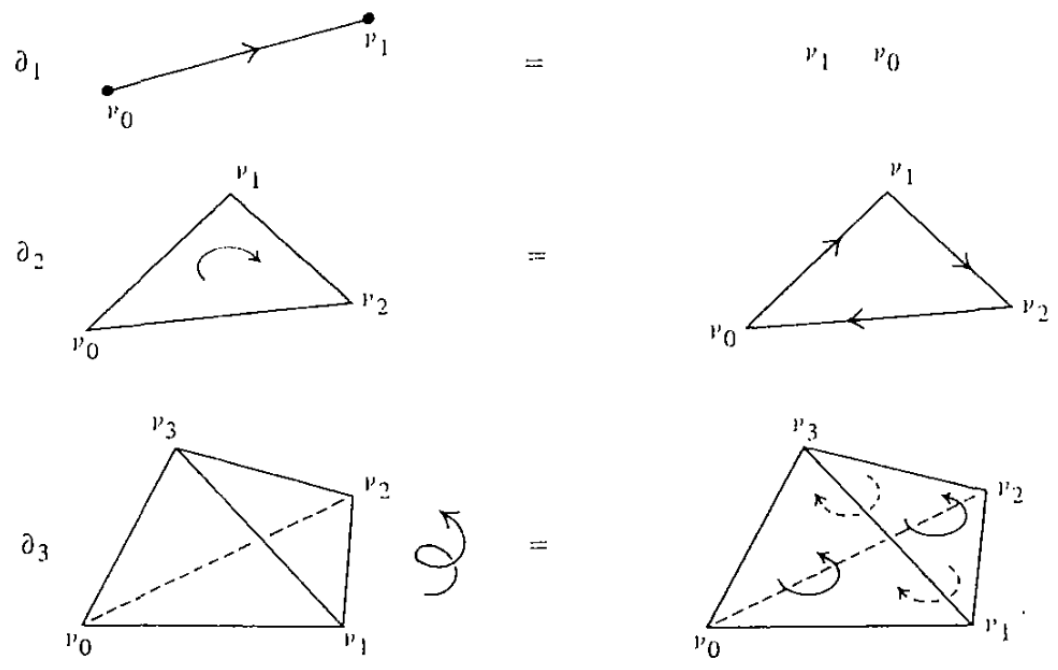

Figura 2.8: Orientação de um simplexo. (Munkres (1984))

\subsubsection{Grupo das $p$-cadeias Simpliciais:}

Definição 2.2.4 Seja $K$ um complexo simplicial. Para o grupo abeliano livre gerado pelos psimplexos orientados de $K, \bar{C}_{p}=\left\{\sum_{\sigma} n_{\sigma} \sigma\right.$ (finita) $\left.\mid \sigma=\left[v_{0}, \ldots, v_{p}\right], n_{\sigma} \in \mathbb{Z}\right\}$, definiremos o grupo gerado pelas $p$-cadeias simpliciais de $K$, orientadas positivamente, como o conjunto $C_{p}(K)=$ $\bar{C}_{p}(K) / \sim$, onde $(-1) \sigma=-\sigma$.

Definição 2.2.5 (Homomorfismo de bordo) Definimos para $C_{p}(K)$ o homomorfismo de bordo como a aplicação

$$
\begin{gathered}
\partial=\partial_{p}: C_{p}(K) \rightarrow C_{p-1}(K) \\
\partial_{p}\left(\left[v_{0}, \ldots, v_{p}\right]\right)=\sum_{i=0}^{p}(-1)^{i}\left[v_{0}, \ldots, \widehat{v}_{i} \ldots, v_{p}\right]
\end{gathered}
$$

onde o vértice $v_{i}$ é omitido.

Definição 2.2.6 Uma p-cadeia $c \in C_{p}(K)$ será chamada de ciclo (ou p-ciclo) se $\partial c=0$. Se $c=\partial_{p+1}\left(c^{\prime}\right)$, para algum $c^{\prime} \in C_{p+1}(K)$, então c será chamada bordo (ou p-bordo). 


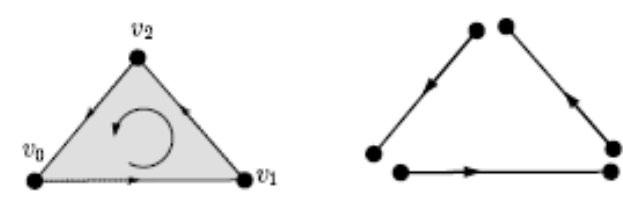

$$
\left[v_{0}, v_{1}, v_{2}\right] \stackrel{\partial}{\longrightarrow}\left[v_{1}, v_{2}\right]-\left[v_{0}, v_{2}\right]+\left[v_{0}, v_{1}\right]
$$
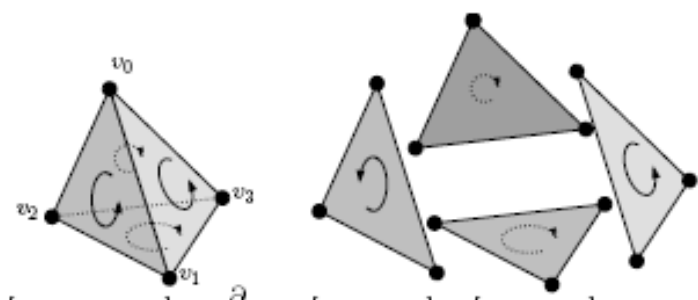

$\left[v_{0}, v_{1}, v_{2}, v_{3}\right] \stackrel{\partial}{\longrightarrow}\left[v_{1}, v_{2}, v_{3}\right]-\left[v_{0}, v_{2}, v_{3}\right]+\ldots$

$\ldots+\left[v_{0}, v_{1}, v_{3}\right]-\left[v_{0}, v_{1}, v_{2}\right]$

Figura 2.9: Bordo para um 2 e 3-simplexo

\subsubsection{Complexo de Cadeias Simpliciais:}

Definição 2.2.7 O complexo de cadeias simpliciais de $K$, denotado por $\left(C_{*}(K), \partial_{*}\right)$, é a sequência de grupos de cadeias simpliciais de $K$ e morfismos entre elas,

$$
\ldots \stackrel{\partial_{p+2}}{\longrightarrow} C_{p+1}(K) \stackrel{\partial_{p+1}}{\longrightarrow} C_{p}(K) \stackrel{\partial_{p}}{\longrightarrow} C_{p-1}(K) \rightarrow \ldots \stackrel{\partial_{1}}{\longrightarrow} C_{0}(K) \stackrel{\partial_{0}}{\longrightarrow} 0
$$

Lema 2.2.1 Se $\partial_{p-1} \circ \partial_{p} \equiv 0, \forall p$.

Demonstração 2.2.1.1

$$
\begin{gathered}
\partial_{p-1} \circ \partial_{p}\left[v_{0}, \ldots, v_{p}\right]=\sum_{i=0}^{p}(-1)^{i} \partial_{p-1}\left[v_{0}, \ldots, \widehat{v}_{i}, \ldots, v_{p}\right] \\
=\sum_{i=0}^{p}\left(\sum_{j=0}^{i-1}(-1)^{i+j}\left[v_{0}, \ldots, \widehat{v_{j}}, \ldots, \widehat{v}_{i}, \ldots, v_{p}\right]+\sum_{j=i+1}^{p}(-1)^{i+j-1}\left[v_{0}, \ldots, \widehat{v}_{i}, \ldots, \widehat{v}_{j}, \ldots, v_{p}\right]\right) \\
=\sum_{i<j}(-1)^{i+j}\left[v_{0}, \ldots, \widehat{v_{j}}, \ldots, \widehat{v}_{i}, \ldots, v_{p}\right]+\sum_{i>j}(-1)^{i+j-1}\left[v_{0}, \ldots, \widehat{v}_{i}, \ldots, \widehat{v_{j}}, \ldots, v_{p}\right]=0
\end{gathered}
$$

Observação 2.2.1 Utilizamos o fato de que para um p-simplexo orientado $\sigma=\left[v_{0}, \ldots, v_{p}\right]$, toda face $(p-2)$-dimensional $\tau$ pertence a duas faces $(p-1)$-dimensionais com orientações induzidas por $\sigma$, que por seguinte induzem orientações opostas em $\tau$ (Lima (2012), Teorema 3, pag. 91), justificando o cancelamento dos pares no somatório final da demonstração.

Segue do lema que $\operatorname{Im}\left(\partial_{p}\right) \subset \operatorname{Ker}\left(\partial_{p-1}\right)$.

Definição 2.2.8 Para cada homomorfismo $\partial$ de cadeias simpliciais, definimos os subgrupos $Z_{p}(K)$ (ciclos) e $B_{p}(K)$ (bordos) de $C_{p}(K)$ com sendo, $Z_{p}(K)=K \operatorname{Ker}\left(\partial_{p}\right)$ e $B_{p}(K)=\operatorname{Im}\left(\partial_{p+1}\right)$.

\subsubsection{Grupos de Homologia Simplicial:}

Definição 2.2.9 Para $\left(C_{*}(K, \mathbb{Z}), \partial_{*}\right)$ complexo de cadeias, definimos sua homologia simplicial como sendo o grupo

$$
H_{p}(K, \mathbb{Z})=\frac{K \operatorname{er}\left\{\partial: C_{p}(K, \mathbb{Z}) \rightarrow C_{p-1}(K, \mathbb{Z})\right\}}{\operatorname{Im}\left\{\partial: C_{p+1}(K, \mathbb{Z}) \rightarrow C_{p}(K, \mathbb{Z})\right\}}=\frac{Z_{p}(K, \mathbb{Z})}{B_{p}(K, \mathbb{Z})}
$$


Os elementos de $H_{p}(K, \mathbb{Z})$ são classes de equivalência de ciclos: ciclos serão equivalentes se a diferença entre eles for um bordo e neste caso serão chamados de ciclos homólogos, isto é, $c, c^{\prime}$ são homólogos em $C_{p}(K, \mathbb{Z})$ se existe uma $(p+1)$-cadeia d tal que $\partial_{p+1} d=c-c^{\prime}$.

Por definição, se um complexo simplicial $K$ não possui $p$-simplexos, então $H_{p}(K, \mathbb{Z})=0$.

Iremos agora calcular alguns grupos de homologia simplicial para determinados espaços topológicos.

Proposição 2.2.1 Seja $K$ um complexo simplicial. Então $H_{0}(K, \mathbb{Z})=\mathbb{Z} \Longleftrightarrow|K|$ é conexo.

Demonstração 2.2.1.1 Seja $V$ o conjunto dos vértices de $K$. Primeiramente, observamos que:

(1) Todo $v \in V$ é um ciclo. (Pois $\partial v=0)$;

(2) Nenhum $v \in V$ é bordo: As 1-cadeias são da forma $\left[v_{i}, v_{j}\right]$ e portanto $\partial\left[v_{i}, v_{j}\right]=v_{j}-v_{i}$ e portanto $[v] \in H_{0}(K, \mathbb{Z})$;

(3) $|K|$ é conexo $\Rightarrow[v]=[w], \forall v, w \in V$ pois, existem $v_{0}, \ldots, v_{k}$, com $v_{0}=v$ e $v_{k}=w \quad e$ $\left(v_{i}, v_{i+i}\right) \in K \Rightarrow \partial\left(\left[v_{0}, v_{1}\right]+\ldots+\left[v_{k-1}, v_{k}\right]\right)=v_{1}-v_{0}+v_{2}-v_{1}+\ldots+v_{k}-v_{k-1}=v_{k}-v_{0}=w-v$.

(4) Se $[v]=[w], \forall v, w \in V \Rightarrow|K|$ é conexo, pois se $|K|$ não for conexo $\Rightarrow \exists w$ tal que $v, w$ não podem ser ligados por 1-simplexos $\Rightarrow[v] \neq[w]$.

Segue que $|K|$ é conexo $\Longleftrightarrow \forall z \in C_{0}(K, \mathbb{Z}), z \sim$ nw para algum $n \in \mathbb{Z}$.

Existe, portanto, existe um isomorfismo $\phi$

$$
\begin{array}{cl}
\phi: H_{0}(K, \mathbb{Z}) & \longrightarrow \mathbb{Z} \\
n[v] & \longmapsto n
\end{array}
$$

$\operatorname{Logo}, H_{0}(K, \mathbb{Z}) \approx \mathbb{Z}$

A ideia intuitiva para o cálculo da homologia simplicial será apresentada nos exemplos a seguir (Ver. Gallier e Quaintance (2016)). Utilizaremos o resultado anterior para este cálculo. Na figura

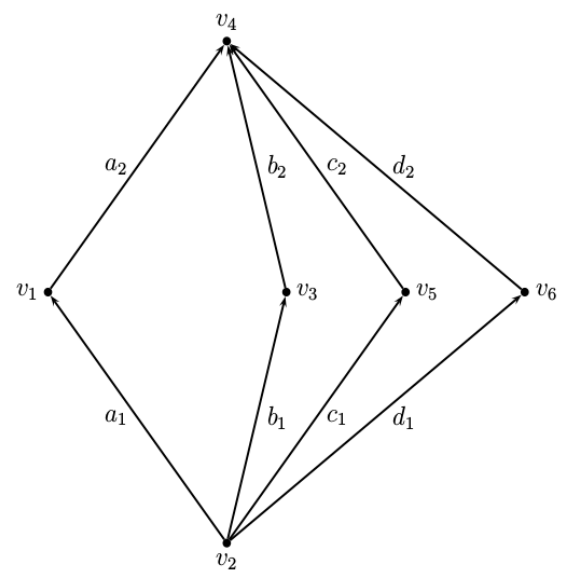

Figura 2.10: Complexo Simplicial $K_{1}\left(\operatorname{dim} K_{1}=1\right)$ (Gallier e Quaintance (2016)).

2.10 um complexo simplicial formado por 6 "vértices" (0-simplexos): $v_{1}, \ldots, v_{6}$ e 8 "arestas" (1simplexos): $a_{1}, a_{2}, b_{1}, b_{2}, c_{1}, c_{2}, d_{1}, d_{2}$ orientados (sentido das setas) tais que:

$$
\begin{array}{cccc}
a_{1}=\left[v_{2}, v_{1}\right] & a_{2}=\left[v_{1}, v_{4}\right] & b_{1}=\left[v_{2}, v_{3}\right] & b_{2}=\left[v_{3}, v_{4}\right] \\
c_{1}=\left[v_{2}, v_{5}\right] & c_{2}=\left[v_{5}, v_{4}\right] & d_{1}=\left[v_{2}, v_{6}\right] & d_{2}=\left[v_{6}, v_{4}\right] \\
\ldots \stackrel{\partial_{3}}{\longrightarrow} 0 \stackrel{\partial_{2}}{\longrightarrow} C_{1}\left(K_{1}, \mathbb{Z}\right) \stackrel{\partial_{1}}{\longrightarrow} C_{0}\left(K_{1}, \mathbb{Z}\right) \stackrel{\partial_{0}}{\longrightarrow} 0 .
\end{array}
$$


Uma vez que este complexo é conexo formado apenas por simplexos de dimensão $p=0,1 \mathrm{e}$ pelo resultado anteriormente apresentado, esperamos que $H_{0}(K, \mathbb{Z})=\mathbb{Z}$. Afirmamos este fato pois, dados dois vértices $u, v \in K_{1}$, existe um caminho $\alpha: I \rightarrow K_{1}$, tal que $\alpha(0)=u$ e $\alpha(1)=v$, e este segue através dos vértices em $K_{1}$, portanto $\partial(\alpha)=u-v \Rightarrow u$ e $v$ são homólogos em $K_{1}$. Em consequência, qualquer $c \in C_{0}\left(K_{1}, \mathbb{Z}\right)\left(0\right.$-cadeia) é equivalente a $\left(\sum n_{i}\right) v_{0}$, portanto:

$$
H_{0}\left(K_{1}, \mathbb{Z}\right)=\left\langle v_{0}\right\rangle \approx \mathbb{Z}
$$

Dado um 1-simplexo $w=\left[v_{i}, v_{j}\right] \in K_{1} \Rightarrow \partial_{1} w=v_{j}-v_{i}$, olhando para o conjunto $Z_{1}\left(K_{1}, \mathbb{Z}\right)$, concluímos que seus representantes principais são os ciclos:

$$
x=a_{1}+a_{2}-b_{2}-b_{1} \quad y=b_{1}+b_{2}-c_{2}-c_{1} \quad z=c_{1}+c_{2}-d_{2}-d_{1}
$$

Estas 1 -cadeias $($ ciclos $)$ em $C_{1}\left(K_{1}, \mathbb{Z}\right)$ formam uma base para $Z_{1}\left(K_{1}, \mathbb{Z}\right)=K \operatorname{Ker}\left(\partial_{1}\right)$, segue portanto:

$$
H_{1}\left(K_{1}, \mathbb{Z}\right)=K e r \partial_{1} / \operatorname{Im}_{2}=K e r \partial_{1}=\langle x, y, z\rangle \approx \mathbb{Z} \oplus \mathbb{Z} \oplus \mathbb{Z} .
$$

Este resultado nos mostra que $K_{1}$ apresenta 3 "buracos" unidimensionais, que facilmente vemos na figura 2.10 .

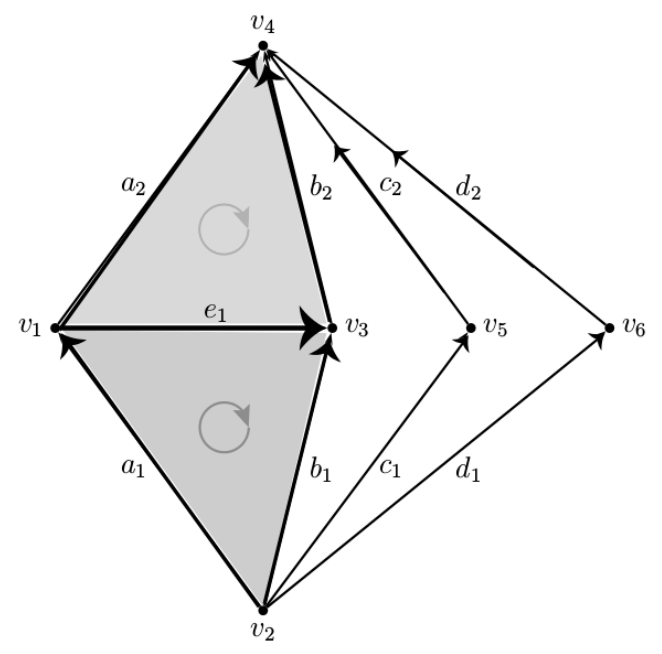

Figura 2.11: Complexo Simplicial $K_{2}\left(\operatorname{dim} K_{2}=2\right)$ (Gallier e Quaintance (2016)).

Agora, consideremos a figura 2.11 e nela apresentamos $K_{2}=K_{1} \cup\left[v_{2}, v_{1}, v_{3}\right] \cup\left[v_{1}, v_{4}, v_{3}\right]$, formado pela adição de mais um 1-simplexo $e_{1}=\left[v_{1}, v_{3}\right]$ que completa os dois 2-simplexos, $A_{1}=\left[v_{2}, v_{1}, v_{3}\right]$ e $A_{2}=\left[v_{1}, v_{4}, v_{3}\right]$ (Orientação destacada na figura $\circlearrowright / \circlearrowleft$ ). Podemos escrever:

$$
\cdots \stackrel{\partial_{3}}{\longrightarrow} C_{2}\left(K_{2}, \mathbb{Z}\right) \stackrel{\partial_{2}}{\longrightarrow} C_{1}\left(K_{2}, \mathbb{Z}\right) \stackrel{\partial_{1}}{\longrightarrow} C_{0}\left(K_{2}, \mathbb{Z}\right) \stackrel{\partial_{0}}{\longrightarrow} 0 .
$$

Para os simplexos orientados $A_{1}$ e $A_{2}$ teremos:

$$
\partial_{2} A_{1}=a_{1}+e_{1}-b_{1} \quad \partial_{2} A_{2}=a_{2}-b_{2}-e_{1}
$$

Segue que

$$
\partial_{2}\left(A_{1}+A_{2}\right)=\partial_{2} A_{1}+\partial_{2} A_{2}=a_{1}+\alpha_{1}-b_{1}+a_{2}-b_{2}-\alpha_{1}=a_{1}+a_{2}-b_{1}-b_{2} .
$$

Portanto não existem 2-ciclos em $C_{2}\left(K_{2}, \mathbb{Z}\right) \Rightarrow Z_{2}\left(K_{2}, \mathbb{Z}\right)=\{0\} \Rightarrow H_{2}\left(K_{2}, \mathbb{Z}\right)=0$.

Considerando, agora, o complexo simplicial $K_{3}$ da figura 2.12. Este novo complexo consiste em 8 vértices (0-simplexos) $\left\{V_{1}, \ldots, v_{8}\right\}, 16$ arestas orientadas, a saber: 


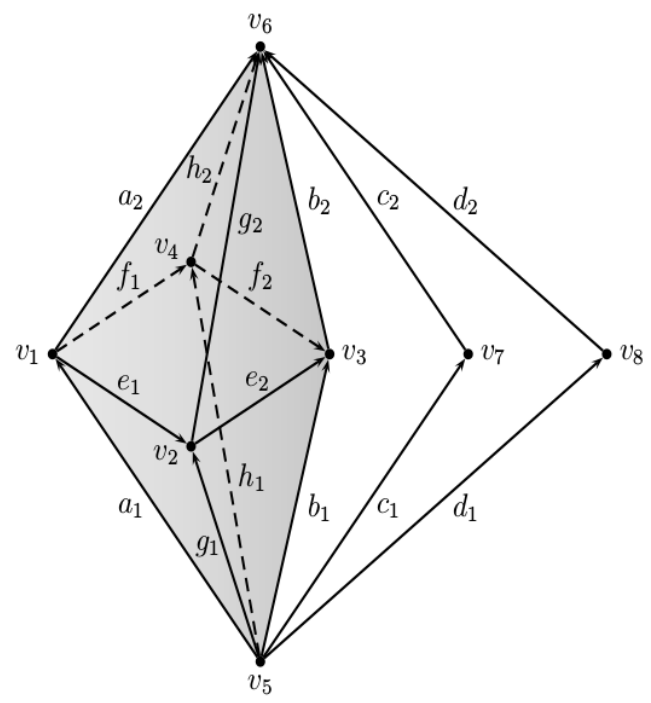

Figura 2.12: Complexo Simplicial $K_{3}\left(\operatorname{dim} K_{3}=2\right)$ (Gallier e Quaintance (2016)).

$$
\begin{array}{llll}
a_{1}=\left[v_{5}, v_{1}\right] & a_{2}=\left[v_{1}, v_{6}\right] & b_{1}=\left[v_{5}, v_{3}\right] & b_{2}=\left[v_{3}, v_{6}\right] \\
c_{1}=\left[v_{5}, v_{7}\right] & c_{2}=\left[v_{4}, v_{6}\right] & d_{1}=\left[v_{5}, v_{8}\right] & d_{2}=\left[v_{8}, v_{6}\right] \\
e_{1}=\left[v_{1}, v_{2}\right] & e_{2}=\left[v_{2}, v_{3}\right] & f_{1}=\left[v_{1}, v_{4}\right] & f_{2}=\left[v_{4}, v_{3}\right] \\
g_{1}=\left[v_{5}, v_{2}\right] & g_{2}=\left[v_{2}, v_{6}\right] & h_{1}=\left[v_{5}, v_{4}\right] & h_{2}=\left[v_{4}, v_{6}\right]
\end{array}
$$

E 8 triângulos (faces) orientados (2-simplexos):

$$
\begin{array}{llll}
A_{1}=\left[v_{5}, v_{1}, v_{2}\right] & A_{2}=\left[v_{5}, v_{2}, v_{3}\right] & A_{3}=\left[v_{1}, v_{6}, v_{2}\right] & A_{4}=\left[v_{2}, v_{6}, v_{3}\right] \\
B_{1}=\left[v_{5}, v_{1}, v_{4}\right] & B_{2}=\left[v_{5}, v_{4}, v_{3}\right] & B_{3}=\left[v_{1}, v_{6}, v_{4}\right] & B_{4}=\left[v_{4}, v_{6}, v_{3}\right]
\end{array}
$$

E assim, para o complexo de cadeias simpliciais de $K_{3}$,

$$
\cdots \stackrel{\partial_{3}}{\longrightarrow} C_{2}\left(K_{3}, \mathbb{Z}\right) \stackrel{\partial_{2}}{\longrightarrow} C_{1}\left(K_{3}, \mathbb{Z}\right) \stackrel{\partial_{1}}{\longrightarrow} C_{0}\left(K_{3}, \mathbb{Z}\right) \stackrel{\partial_{0}}{\longrightarrow} 0 .
$$

Podemos calcular o bordo de cada 2-simplexo, lembrando que para todo p-simplexos temos:

$$
\partial(A)=\sum_{i=0}^{p}(-1)^{i}\left[v_{0}, \ldots, \widehat{v}_{i}, \ldots, v_{p}\right]
$$

Para $A_{1}$, teremos:

$$
\begin{aligned}
\partial_{2}\left(A_{1}\right) & =(-1)^{0}\left[\widehat{v}_{5}, v_{1}, v_{2}\right]+(-1)^{1}\left[v_{5}, \widehat{v}_{1}, v_{2}\right]+(-1)^{2}\left[v_{5}, v_{1}, \widehat{v}_{2}\right] \\
& =\left[v_{1}, v_{2}\right]-\left[v_{5}, v_{2}\right]+\left[v_{5}, v_{1}\right] \\
& =e_{1}-g_{1}+a_{1}=a_{1}+e_{1}-g_{1}
\end{aligned}
$$

E, de maneira análoga, teremos:

$$
\begin{array}{llll}
\partial_{2}\left(A_{1}\right)=a_{1}+e_{1}-g_{1} & \partial_{2}\left(A_{2}\right)=g_{1}+e_{2}-b_{1} & \partial_{2}\left(A_{3}\right)=a_{2}-g_{2}-e_{1} & \partial_{2}\left(A_{4}\right)=g_{2}-b_{2}-e_{2} \\
\partial_{2}\left(B_{1}\right)=a_{1}-f_{1}-h_{1} & \partial_{2}\left(B_{2}\right)=h_{1}+f_{2}-b_{1} & \partial_{2}\left(B_{3}\right)=a_{2}-h_{2}-f_{1} & \partial_{2}\left(B_{4}\right)=h_{2}-b_{2}-f_{2} .
\end{array}
$$


Se fizermos:

$$
A=A_{1}+A_{2}+A_{3}+A_{4} \text { e } B=B_{1}+B_{2}+B_{3}+B_{4}\left(\mathrm{em} C_{2}\left(K_{3}, \mathbb{Z}\right)\right) .
$$

Então,

$$
\partial_{2}(A)=\partial_{2}(B)
$$

Pois,

$$
\begin{aligned}
& \partial_{2}(A)=\left(a_{1}+e_{1}-g_{1}\right)+\left(g_{1}+e_{2}-b_{1}\right)+\left(a_{2}-g_{2}-e_{1}\right)+\left(g_{2}-b_{2}-e_{2}\right) \\
& =a_{1}+e_{1}-y_{1}+y_{1}+x_{2}-b_{1}+a_{2}-g_{2}-e_{1}+g_{2}-b_{2}-x_{2} \\
& =\left(a_{1}+a_{2}\right)-\left(b_{1}+b_{2}\right) \text {. } \\
& \partial_{2}(B)=\left(a_{1}-f_{1}-h_{1}\right)+\left(h_{1}+f_{2}-b_{1}\right)+\left(a_{2}-h_{2}-f_{1}\right)+\left(h_{2}-b_{2}-f_{2}\right) \\
& =a_{1}-\mathfrak{x}_{1}-\not h_{1}+\not h_{1}+f_{2}-b_{1}+a_{2}-\not h_{2}-\mathfrak{x}_{1}+\not b_{2}-b_{2}-f_{2} \\
& =\left(a_{1}+a_{2}\right)-\left(b_{1}+b_{2}\right) \text {. }
\end{aligned}
$$

Segue que,

$$
\partial_{2}(B-A)=0
$$

Então $D=B-A$ é um 2-ciclo, que neste caso representa um octaedro, ver figura 2.12.

Observamos que o grupo das cadeias simpliciais $C_{2}\left(K_{3}, \mathbb{Z}\right)$ é um grupo abeliano composto por todas as combinações lineares dos $A_{i}^{\prime} s$ e $B_{j}^{\prime} s$ e pelo fato de que $\partial_{2}(B-A)=0$, segue que o núcleo da aplicação bordo

$$
\partial_{2}: C_{2}\left(K_{3}, \mathbb{Z}\right) \rightarrow C_{1}\left(K_{3}, \mathbb{Z}\right)
$$

é não trivial, $\log$ $B-A$ gera um grupo de homologia,

$$
H_{2}\left(K_{3}, \mathbb{Z}\right)=\operatorname{Ker}\left(\partial_{2}\right)=\langle B-A\rangle \approx \mathbb{Z} .
$$

Isto mostra que $K_{3}$ possue apenas um buraco bidimensional e pelos casos anteriores,

$$
H_{1}\left(K_{3}, \mathbb{Z}\right)=\frac{\operatorname{Ker}\left(\partial_{1}\right)}{\operatorname{Im}\left(\partial_{2}\right)} \approx \mathbb{Z} \oplus \mathbb{Z}
$$

O $K_{3}$ também possue 2 buracos unidimensionais e como é conexo segue que $H_{0}\left(K_{3}\right)=\mathbb{Z}$.

Finalmente, passamos agora a analisar o 3-complexo simplicial $K_{4}$ destacado na figura 2.13 , obtido de $K_{3}$ com junção de uma aresta orientada $k=\left[v_{2}, v_{4}\right]$ e 4 tetraedros orientados (3-simplexos):

$$
\begin{array}{rlrl}
T_{1}=\left[v_{1}, v_{2}, v_{4}, v_{6}\right] & & T_{2}=\left[v_{3}, v_{4}, v_{2}, v_{6}\right] \\
T_{3}=\left[v_{1}, v_{4}, v_{2}, v_{5}\right] & & T_{4}=\left[v_{3}, v_{2}, v_{4}, v_{5}\right] \\
\cdots \stackrel{\partial_{4}}{\longrightarrow} C_{3}\left(K_{4}, \mathbb{Z}\right) \stackrel{\partial_{3}}{\longrightarrow} C_{2}\left(K_{4}, \mathbb{Z}\right) \stackrel{\partial_{2}}{\longrightarrow} C_{1}\left(K_{4}, \mathbb{Z}\right) \stackrel{\partial_{1}}{\longrightarrow} C_{0}\left(K_{4}, \mathbb{Z}\right) \stackrel{\partial_{0}}{\longrightarrow} 0 .
\end{array}
$$

Para

$$
\begin{gathered}
\partial_{3}: C_{3}\left(K_{4}, \mathbb{Z}\right) \rightarrow C_{2}\left(K_{4}, \mathbb{Z}\right) \\
\partial_{3}\left(T_{1}\right)=(-1)^{0}\left[\widehat{v}_{1}, v_{2}, v_{4}, v_{6}\right]+(-1)^{1}\left[v_{1}, \widehat{v}_{2}, v_{4}, v_{6}\right]+(-1)^{2}\left[v_{1}, v_{2}, \widehat{v}_{4}, v_{6}\right]+(-1)^{3}\left[v_{1}, v_{2}, v_{4}, \widehat{v}_{6}\right] \\
=\left[v_{2}, v_{4}, v_{6}\right]-\left[v_{1}, v_{4}, v_{6}\right]+\left[v_{1}, v_{2}, v_{6}\right]-\left[v_{1}, v_{2}, v_{4}\right] .
\end{gathered}
$$


E portanto,

$$
\begin{aligned}
& \partial_{3}\left(T_{1}\right)=\left[v_{2}, v_{4}, v_{6}\right]-\left[v_{1}, v_{4}, v_{6}\right]+\left[v_{1}, v_{2}, v_{6}\right]-\left[v_{1}, v_{2}, v_{4}\right] \\
& \partial_{3}\left(T_{2}\right)=\left[v_{4}, v_{2}, v_{6}\right]-\left[v_{3}, v_{2}, v_{6}\right]+\left[v_{3}, v_{4}, v_{6}\right]-\left[v_{3}, v_{4}, v_{2}\right] \\
& \partial_{3}\left(T_{3}\right)=\left[v_{4}, v_{2}, v_{5}\right]-\left[v_{1}, v_{2}, v_{5}\right]+\left[v_{1}, v_{4}, v_{5}\right]-\left[v_{1}, v_{4}, v_{2}\right] \\
& \partial_{3}\left(T_{4}\right)=\left[v_{2}, v_{4}, v_{5}\right]-\left[v_{3}, v_{4}, v_{5}\right]+\left[v_{3}, v_{2}, v_{5}\right]-\left[v_{3}, v_{2}, v_{4}\right] .
\end{aligned}
$$

Observamos que

$$
\begin{aligned}
\partial_{3}\left(T_{1}+T_{2}+T_{3}+T_{4}\right) & =\partial_{3}\left(T_{1}\right)+\partial_{3}\left(T_{2}\right)+\partial_{3}\left(T_{3}\right)+\partial_{3}\left(T_{4}\right) \\
& \left.=\left[v_{2}, v_{4}, v_{6}\right]-\left[v_{1}, v_{4}, v_{6}\right]+\left[v_{1}, v_{2}, v_{6}\right]-v_{1}, v_{4}\right] \\
& \left.+\left[v_{4}, v_{2}, v_{6}\right]-\left[v_{3}, v_{2}, v_{6}\right]+\left[v_{3}, v_{4}, v_{6}\right]-v_{3}, v_{4}, v_{2}\right] \\
& \left.+\left[v_{4}, v_{2}, v_{5}\right]-\left[v_{1}, v_{2}, v_{5}\right]+\left[v_{1}, v_{4}, v_{5}\right]-v_{1}, v_{2}\right] \\
& +\left[v_{2}, v_{4}, v_{5}\right]-\left[v_{3}, v_{4}, v_{5}\right]+\left[v_{3}, v_{2}, v_{5}\right]-\left[v_{3}, v_{2}, v_{4}\right] \\
& =-\left[v_{1}, v_{4}, v_{6}\right]+\left[v_{1}, v_{2}, v_{6}\right]-\left[v_{3}, v_{2}, v_{6}\right]+\left[v_{3}, v_{4}, v_{6}\right] \\
& -\left[v_{1}, v_{4}, v_{5}\right]+\left[v_{1}, v_{4}, v_{5}\right]-\left[v_{3}, v_{4}, v_{5}\right]+\left[v_{3}, v_{2}, v_{5}\right] \\
& =B_{3}-A_{3}-A_{4}+B_{4}-A_{1}+B_{1}+B_{2}-A_{2} \\
& =B_{1}+B_{2}+B_{3}+B_{4}-A_{1}-A_{2}-A_{3}-A_{4} \\
& =\left(B_{1}+B_{2}+B_{3}+B_{4}\right)-\left(A_{1}+A_{2}+A_{3}+A_{4}\right) \\
& =B-A .
\end{aligned}
$$

Segue que a aplicação

$$
\partial_{3}: C_{3}\left(K_{4}, \mathbb{Z}\right) \rightarrow C_{2}\left(K_{4}, \mathbb{Z}\right)
$$

associa o octaedro sólido $T=T_{1}+T_{2}+T_{3}+T_{4}$ para $B-A$ e portanto $\operatorname{Ker}\left(\partial_{2}\right)$ é gerado por $B-A$ e assim,

$$
H_{2}\left(K_{4}, \mathbb{Z}\right)=\frac{\operatorname{Ker}\left(\partial_{2}\right)}{\operatorname{Im}\left(\partial_{3}\right)}=0
$$

E também,

$$
H_{3}\left(K_{4}, \mathbb{Z}\right)=\frac{K e r\left(\partial_{3}\right)}{\operatorname{Im}\left(\partial_{4}\right)}=\operatorname{Ker}\left(\partial_{3}\right)=0 .
$$

E como anteriomente, $H_{0}\left(K_{4}, \mathbb{Z}\right)=\mathbb{Z}$ e $H_{1}\left(K_{4}, \mathbb{Z}\right)=\mathbb{Z} \oplus \mathbb{Z}$. O complexo $K_{4}$ continua tendo um buraco unidimensional mas os dois buracos bidimensionais de $K_{3}$ "somem", uma vez que o octaedro é sólido.

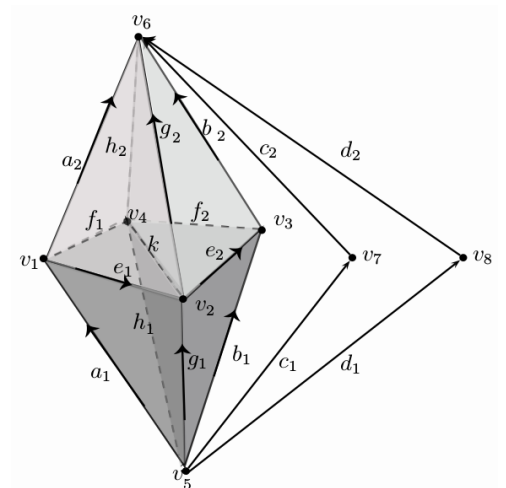

Figura 2.13: Complexo Simplicial $K_{4}\left(\operatorname{dim} K_{4}=3\right)$ (Gallier e Quaintance (2016)). 


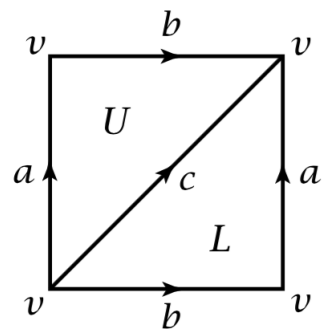

Figura 2.14: $\Delta$-simplexo para o toro $\mathbb{T}^{2}$, (Hatcher (2001)).

$$
\ldots \stackrel{\partial_{p+2}}{\longrightarrow} C_{p+1}\left(\mathbb{T}^{2}, \mathbb{Z}\right) \stackrel{\partial_{p+1}}{\longrightarrow} C_{p}\left(\mathbb{T}^{2}, \mathbb{Z}\right) \stackrel{\partial_{p}}{\longrightarrow} C_{p-1}\left(\mathbb{T}^{2}, \mathbb{Z}\right) \rightarrow \ldots \stackrel{\partial_{1}}{\longrightarrow} C_{0}\left(\mathbb{T}^{2}, \mathbb{Z}\right) \stackrel{\partial_{0}}{\longrightarrow} 0
$$

$\mathrm{Na}$ representação do espaço $\mathbb{T}^{2}$ (figura 2.14) existem apenas: 0-simplexo $(v)$, 1-simplexos $(a, b, c) \mathrm{e}$ 2-simplexos $(U, L)$, desta forma tem-se $C_{p}\left(\mathbb{T}^{2}, \mathbb{Z}\right)=0, \forall p>2$ :

$$
\begin{gathered}
\ldots \stackrel{\partial_{4}}{\longrightarrow} C_{3}\left(\mathbb{T}^{2}, \mathbb{Z}\right) \stackrel{\partial_{3}}{\longrightarrow} C_{2}\left(\mathbb{T}^{2}, \mathbb{Z}\right) \stackrel{\partial_{2}}{\longrightarrow} C_{1}\left(\mathbb{T}^{2}, \mathbb{Z}\right) \stackrel{\partial_{1}}{\longrightarrow} C_{0}\left(\mathbb{T}^{2}, \mathbb{Z}\right) \stackrel{\partial_{0}}{\longrightarrow} 0 \\
\ldots \stackrel{\partial_{4}}{\longrightarrow} 0 \stackrel{\partial_{3}}{\longrightarrow}\langle U, L\rangle \stackrel{\partial_{2}}{\longrightarrow}\langle a, b, c\rangle \stackrel{\partial_{1}}{\longrightarrow}\langle v\rangle \stackrel{\partial_{0}}{\longrightarrow} 0
\end{gathered}
$$

Como $\partial_{1} v=0, \partial_{1} a=\partial_{1} b=\partial_{1} c=v-v=0$ e $\partial_{2} U=\partial_{2} L=a+b-c$.

Para dimensões mais altas $(p \geq 3)$ não temos $p$-simplexos e portanto, $H_{p}\left(\mathbb{T}^{2}, \mathbb{Z}\right)=0$.

Para o cálculo de $H_{2}\left(\mathbb{T}^{2}, \mathbb{Z}\right)$ :

$\operatorname{Ker}\left(\partial_{2}\right)=\left\{x \in C_{2}\left(\mathbb{T}^{2}, \mathbb{Z}\right) / \partial_{2}(x)=0\right\}$ e $\operatorname{Im}\left(\partial_{3}\right)=\left\{x \in C_{2}\left(\mathbb{T}^{2}, \mathbb{Z}\right) / \exists w \in C_{3}\left(\mathbb{T}^{2}, \mathbb{Z}\right) ; \partial_{3}(w)=x\right\}=\{0\}$

Se $x \in C_{2}\left(\mathbb{T}^{2}, \mathbb{Z}\right) \approx\langle U, L\rangle \Rightarrow x=\alpha U+\beta L \Rightarrow \partial_{2} x=\alpha \partial_{2} U+\beta \partial_{2} L=(\alpha+\beta) \partial_{2} U=(\alpha+\beta)(a+$ $b-c) \Rightarrow x \in \operatorname{Ker}\left(\partial_{2}\right) \Longleftrightarrow \alpha+\beta=0 \Longleftrightarrow \alpha=-\beta \Rightarrow x=\alpha(U-L) \in\langle U-L\rangle \approx \mathbb{Z} \approx \operatorname{Ker}\left(\partial_{2}\right)$.

$$
H_{2}\left(\mathbb{T}^{2}, \mathbb{Z}\right)=\frac{\operatorname{Ker}\left(\partial_{2}\right)}{\operatorname{Im}\left(\partial_{3}\right)} \approx \frac{\mathbb{Z}}{\{0\}} \approx \mathbb{Z}
$$

Para o cálculo de $H_{1}\left(\mathbb{T}^{2}\right)$ :

$$
\operatorname{Ker}\left(\partial_{1}\right)=\left\{x \in C_{1}\left(\mathbb{T}^{2}\right) / \partial_{1}(x)=0\right\} \quad e \quad \operatorname{Im}\left(\partial_{2}\right)=\left\{y \in C_{1}\left(\mathbb{T}^{2}\right) / \exists x \in C_{2}\left(\mathbb{T}^{2}\right) ; \partial_{2}(x)=y\right\}
$$

Seja $x \in C_{2}\left(\mathbb{T}^{2}\right)=\langle U, L\rangle \Rightarrow x=\alpha U+\beta L \Rightarrow \partial_{2}(x)=\alpha \partial_{2} U+\beta \partial_{2} L \Rightarrow \partial_{2}(x)=y=$ $(\alpha+\beta)(a+b-c)=\langle a+b-c\rangle \approx \mathbb{Z} \Rightarrow \operatorname{Im}\left(\partial_{2}\right) \approx \mathbb{Z}$.

Como $\partial a=\partial b=\partial c=0 y \in\langle a, b, c\rangle \Rightarrow y \in \mathbb{Z} \oplus \mathbb{Z} \oplus \mathbb{Z} \Longleftrightarrow \operatorname{Ker}\left(\partial_{1}\right) \approx \mathbb{Z} \oplus \mathbb{Z} \oplus \mathbb{Z}$.

$$
H_{1}\left(\mathbb{T}^{2}\right)=\frac{\operatorname{Ker}\left(\partial_{1}\right)}{\operatorname{Im}\left(\partial_{2}\right)} \approx \frac{\mathbb{Z} \oplus \mathbb{Z} \oplus \mathbb{Z}}{\mathbb{Z}} \approx \frac{\langle a, b, c\rangle}{\langle a+b-c\rangle} \approx \frac{\langle a, b, a+b-c\rangle}{\langle a+b-c\rangle} \approx \mathbb{Z} \oplus \mathbb{Z}
$$

Como $\partial_{0}=0 \Rightarrow \operatorname{Ker}\left(\partial_{0}\right)=C_{1}\left(\mathbb{T}^{2}\right)=\langle v\rangle \approx \mathbb{Z}$ e $\operatorname{Im}\left(\partial_{1}\right)=\{0\}$ segue que:

$$
\begin{array}{r}
H_{0}\left(\mathbb{T}^{2}\right)=\frac{\operatorname{Ker}\left(\partial_{0}\right)}{\operatorname{Im}\left(\partial_{1}\right)} \approx \frac{\mathbb{Z}}{\{0\}} \approx \mathbb{Z} \\
H_{p}\left(\mathbb{T}^{2}\right)=\left\{\begin{array}{cr}
\mathbb{Z} \oplus \mathbb{Z} & \text { se } p=1 \\
\mathbb{Z} & \text { se } p=0,2 \\
0 & \text { se } p \geq 3
\end{array}\right.
\end{array}
$$

Observação 2.2.2 (Cálculo de $H_{p}(K)$ ) Para o cálculo efetivo dos grupos de homologia simplicial, seguiremos os passos a seguir (Kinsey (1993), pp. 131)

(1) Exibimos $C_{p}(K)$ (grupo das $p$-cadeias de $K$ ) para cada $p \geq 0$; 
(2) Para cada p-cadeia geradora $c$ de (1), calculamos $\partial c$.

(3) Encontramos $Z_{p}(K)$ usando os resultados de (2).

(a) Notemos que se $Z_{p}(K)=\{0\}$, então $H_{p}(K)=\{0\}$

(4) Encontramos $B_{p}(K)$.

(a) Quando em dimensões altas, $B_{p}(K)=\emptyset$, uma vez que não existem $(p+1)$-cadeias para as quais existem p-cadeias formando bordo.

(b) Qualquer outro caso, olhamos para o passo (2) onde, em dimensões altas, já calculamos as p-cadeias que são bordos.

(c) Notamos que se $B_{p}(K)=\{0\}$, então $H_{p}(K)=Z_{p}(K)$

(5) Calculamos $H_{p}(K)$ usando (3) e (4).

Tendo definido e calculado alguns grupos de homologia simplicial, relacionaremos os grupos de homologia de um complexo simplicial e sua subdivisão baricêntrica.

\subsubsection{Aplicações de Cadeias:}

Definição 2.2.10 $f:|K| \rightarrow|L|$ é uma aplicação simplicial.

Para cada $p$, a aplicação $f$ induz $f_{*, p}: C_{p}(K) \rightarrow C_{p}(L)$ aplicação de cadeias (homomorfismo de grupos abelianos),

$$
f_{*}\left(\left[v_{\alpha_{0}}, \ldots, v_{\alpha_{p}}\right]\right)=\left\{\begin{array}{c}
{\left[f\left(v_{\alpha_{0}}\right), \ldots, f\left(v_{\alpha_{p}}\right)\right], f\left(v_{\alpha_{i}}\right) \neq f\left(v_{\alpha_{j}}\right), \forall i \neq j} \\
0 \text { caso contrário. }
\end{array}\right.
$$

Lema 2.2.2 $f_{*}: C_{*}(K) \rightarrow C_{*}(L)$ é uma aplicação de cadeias, isto é, $f_{*} \partial=\partial f_{*}$ :

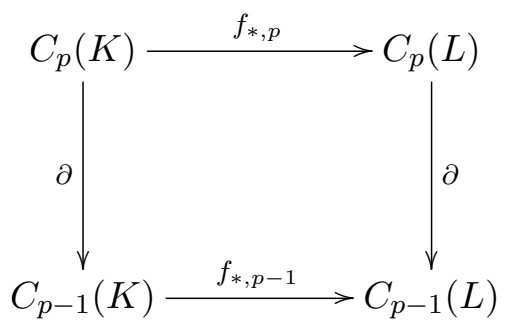

Demonstração 2.2.2.1 Basta verificar que o diagrama acima comuta,

(1) Suponha que $f_{*}\left(v_{i}\right) \neq f\left(v_{j}\right), \forall i, j$, então:

$$
\begin{gathered}
\partial f_{*}\left[v_{0}, \ldots, v_{p}\right]=\partial\left[f\left(v_{0}\right), \ldots, f\left(v_{p}\right)\right]=\sum(-1)^{i}\left[f\left(v_{0}, \ldots, \widehat{f\left(v_{i}\right)}, \ldots, f\left(v_{p}\right)\right]=\right. \\
=f_{*}\left(\sum(-1)^{i}\right)\left[v_{0}, \ldots, \widehat{v}_{i}, \ldots, v_{p}\right]=f_{*} \partial\left[v_{0}, \ldots, v_{p}\right] \Rightarrow f_{*} \partial=\partial f_{*}
\end{gathered}
$$

(2) Supondo agora, $f\left(v_{i}\right)=f\left(v_{j}\right)$ para alguns $i, j$. Sem perda de generalidade, seja $f\left(v_{0}\right)=f\left(v_{1}\right)$ e portanto:

$$
\begin{gathered}
\partial\left[v_{0}, \ldots, v_{p}\right]=\partial 0=0 \\
f_{*} \partial\left[v_{0}, \ldots, v_{p}\right]=f_{*}\left(\sum(-1)^{i}\left[v_{0}, \ldots, \widehat{v}_{i}, \ldots, v_{p}\right]\right)= \\
=\left[f\left(v_{1}\right), \ldots, f\left(v_{p}\right)\right]-\left[f\left(v_{0}\right), f\left(v_{2}\right), \ldots, f\left(v_{p}\right)\right]=0
\end{gathered}
$$


Corolário 2.2.2.1 $f_{*}$ leva ciclo em ciclo e bordo em bordo, pois, $\partial z=0 \Rightarrow \partial f_{*} z=f_{*} \partial z=0$ e $z=\partial w \Rightarrow f_{*} z=f_{*} \partial w=\partial f_{*} w$. Logo, $f$ induz

$$
f_{*}: H_{p}(K) \rightarrow H_{p}(L)
$$

Teorema 2.2.1 (Número de Euler em termos de Homologia Simplicial) A característica de Euler pode ser calculada como sendo:

$$
\chi(K)=\sum_{i=0}^{n}(-1)^{i} \cdot \beta_{i}(X)=\sum_{i=0}^{n}(-1)^{i} \cdot \operatorname{dim}\left(H_{i}(K, \mathbb{R})\right)
$$

Onde $\beta_{i}$ é o número de Betti de $X$.

\section{Demonstração 2.2.1.1 Seja}

$$
0 \rightarrow C_{n}(K, \mathbb{R}) \stackrel{\partial_{n}}{\longrightarrow} C_{n-1}(K, \mathbb{R}) \stackrel{\partial_{n-1}}{\longrightarrow} \ldots \stackrel{\partial_{1}}{\longrightarrow} C_{0}(K, \mathbb{R}) \stackrel{\partial_{0}}{\longrightarrow} 0
$$

um complexo de cadeias de grupos finitamente gerados tais que: $Z_{n}(K, \mathbb{R})=\operatorname{Ker}\left(\partial_{n}\right)$ são seus ciclos e $B_{n}(K, \mathbb{R})=\operatorname{Im}\left(\partial_{n+1}\right)$, seus bordos, teremos,

$$
H_{n}(K, \mathbb{R})=\frac{Z_{n}(K, \mathbb{R})}{B_{n}(K, \mathbb{R})}
$$

E para as sequências exatas

$0 \rightarrow Z_{n}(K, \mathbb{R}) \rightarrow C_{n}(K, \mathbb{R}) \rightarrow B_{n-1}(K, \mathbb{R}) \rightarrow 0$ e $0 \rightarrow B_{n}(K, \mathbb{R}) \rightarrow Z_{n}(K, \mathbb{R}) \rightarrow H_{n}(K, \mathbb{R}) \rightarrow 0$ e em virtude do teorema do núcleo e da imagem, teremos também,

$$
\begin{aligned}
& \operatorname{dim}\left(C_{n}(K, \mathbb{R})\right)=\operatorname{dim}\left(Z_{n}(K, \mathbb{R})\right)+\operatorname{dim}\left(B_{n-1}(K, \mathbb{R})\right) \\
& \operatorname{dim}\left(Z_{n}(K, \mathbb{R})\right)=\operatorname{dim}\left(B_{n}(K, \mathbb{R})\right)+\operatorname{dim}\left(H_{n}(K, \mathbb{R})\right)
\end{aligned}
$$

Substituindo o valor $\operatorname{dim}\left(Z_{n}(K, \mathbb{R})\right)$ da relação 2.2 em 2.1:

$$
\operatorname{dim}\left(C_{n}(K, \mathbb{R})\right)=\operatorname{dim}\left(B_{n}(K, \mathbb{R})\right)+\operatorname{dim}\left(H_{n}(K, \mathbb{R})\right)+\operatorname{dim}\left(B_{n-1}(K, \mathbb{R})\right)
$$

Multiplicando a equação por $(-1)^{n}$, teremos

$(-1)^{n} \operatorname{dim}\left(C_{n}(K, \mathbb{R})\right)=(-1)^{n} \operatorname{dim}\left(B_{n}(K, \mathbb{R})\right)+(-1)^{n} \operatorname{dim}\left(H_{n}(K, \mathbb{R})\right)+(-1)^{n} \operatorname{dim}\left(B_{n-1}(K, \mathbb{R})\right), \forall n$

Somando termo a termo, teremos:

$$
\begin{gathered}
\sum_{i=0}^{n}(-1)^{i} \operatorname{dim}\left(C_{i}(K, \mathbb{R})\right)=\sum_{i=0}^{n}(-1)^{i} \operatorname{dim}\left(B_{i}(K, \mathbb{R})\right)+\sum_{i=0}^{n}(-1)^{i} \operatorname{dim}\left(H_{i}(K, \mathbb{R})\right)+\sum_{i=0}^{n}(-1)^{i} \operatorname{dim}\left(B_{i-1}(K, \mathbb{R})\right) \\
\sum_{i=0}^{n}(-1)^{i} \operatorname{dim}\left(C_{i}(K, \mathbb{R})\right)=\sum_{i=0}^{n}(-1)^{i} \operatorname{dim}\left(H_{i}(K, \mathbb{R})\right) \\
\chi(X)=\sum_{i=0}^{n} \operatorname{dim}\left(H_{i}(K, \mathbb{R})\right) .
\end{gathered}
$$




\subsection{Pseudo - Variedades}

Uma pseudo-variedade é um tipo especial de complexo simplicial, sua estrutura se assemelha à uma variedade na maioria dos pontos, mas pode conter singularidades.

Podemos conceber a ideia de pseudo-variedade com sendo realização combinatória de variedades com singularidades. Os conceitos de orientabilidade fazem sentido assim como em variedades.

Definição 2.3.1 Um complexo simplicial $K$ de dimensão $n$ é uma pseudo-variedade irredutivel se:

(1) $K$ é conexo por $n$-cadeias:

Dados $\sigma, \sigma^{\prime} n$-simplexos de $K$, existe uma sequência de $n$-simplexos $\sigma=\sigma_{0}, \sigma_{1}, \ldots, \sigma_{l}=\sigma^{\prime} \in K$ tais que $\sigma_{i} \cap \sigma_{i+1}$ é um $(n-1)$ - simplexo de $K$.

(2) $K$ é homogêneo: Todo simplexo é face de algum n-simplexo.

(3) Cada $(n-1)$ - simplexo é face de exatamente dois n-simplexos.
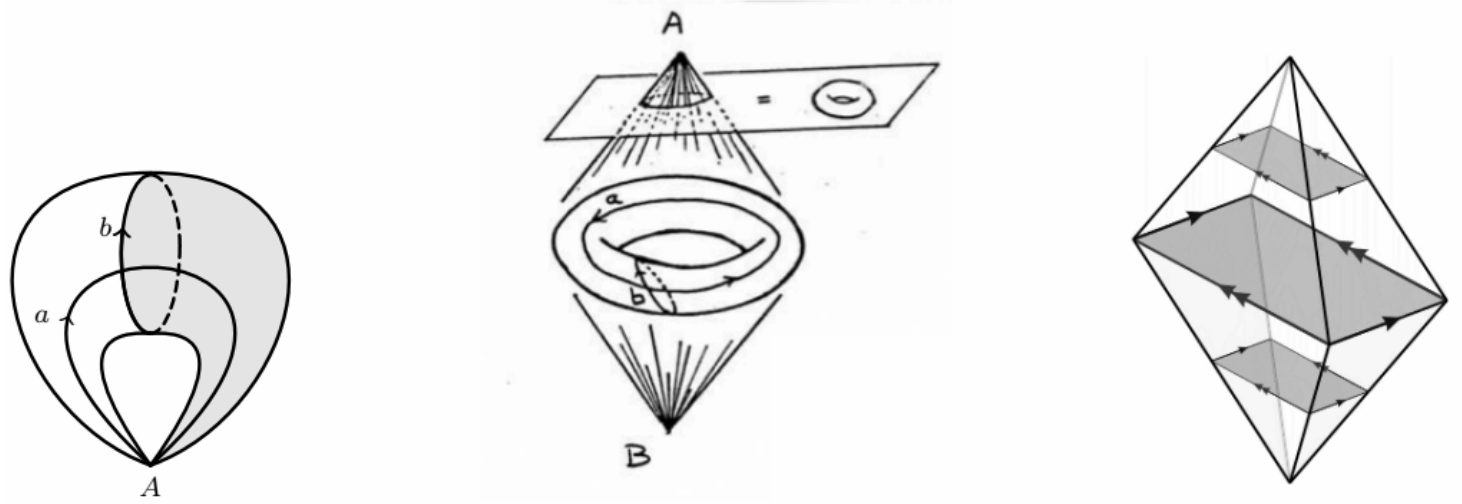

Figura 2.15: Pseudo-variedades.

Definição 2.3.2 Uma pseudo-variedade de dimensão n é um complexo simplicial que é união de pseudo-variedades irredutiveis de dimensão $n$.

\section{Exemplo 2.3.1}

(1) Seja $K \subset \mathbb{R}^{n}$ uma pseudo-variedade. Mergulhando $\mathbb{R}^{n}$ em $\mathbb{R}^{n+1}$ e tomando um ponto a $\epsilon$ $\mathbb{R}^{n+1}-\mathbb{R}^{n}$. A união de todos os segmentos da forma $[a, x]$, onde $x \in K$ é uma $(n+1)$-pseudovariedade, chamada cone de $M$, representada por $\Sigma K$. (Prasolov (2006))

(2) A suspensão $\left(S K=(K \times I) / \sim ;\left(x_{1}, 0\right) \sim\left(x_{2}, 0\right)\right.$ e $\left.\left.\left(x_{1}, 1\right) \sim\left(x_{2}, 1\right), \forall x_{1}, x_{2} \in K\right)\right)$ de uma variedade usual (topológica ou suave), finita $K$ de $\operatorname{dim} K=n$ pode ser uma variedade somente se $K$ possui mesma homologia que da esfera, de outra forma, se K é uma pseudovariedade de dimensão $n$, e $L$ é um $(n-2)$ - esqueleto de $K$, então, $K-L$ é uma variedade. Portanto, qualquer pseudo-variedade se torna uma variedade após remoção de um conjunto de codimensão 2. ( Prasolov (2006))

\subsubsection{Orientação:}

Definição 2.3.3 Seja K uma pseudo-variedade de dimensão n. Sejam $\sigma, \sigma^{\prime} n$-simplexos de $K$, $\sigma \cap \sigma^{\prime}=\tau \neq \emptyset$. Uma orientação em $\sigma$ é coerente com uma orientação em $\sigma^{\prime}$ se as orientações induzidas em $\tau$ são opostas.

Definição 2.3.4 Uma orientação em $K$ é uma escolha de orientação em cada n-simplexo de $K$ tal que se $\sigma \cap \sigma^{\prime} \neq \emptyset$ implicam que as orientações são coerentes. $K$ é orientável se for possível escolher um orientação. Uma pseudo-variedade orientada é uma pseudo-variedade $K$ com uma escolha de orientação. 
Observação 2.3.1 Se K é orientável e irredutivel a escolha de orientação em um n-simplexo determina a orientação em $K$ logo, uma pseudo-variedade irredutível orientável admite duas escolhas de orientaçôes.

Exemplo 2.3.2 (Faixa de Möbius) A faixa de Möbius como um complexo simplicial com seis vértices é uma pseudo-variedade não-orientável.

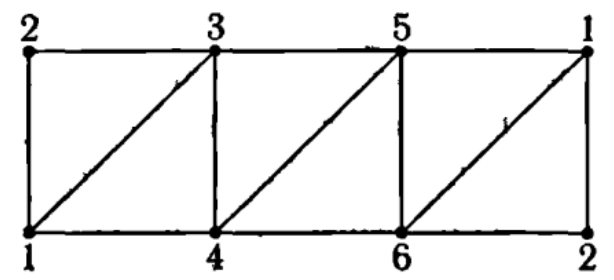

Figura 2.16: Faixa de Möbius como pseudo-variedade.

Teorema 2.3.1 Se K é uma pseudo-variedade fechada, irredutível, não-orientável de dimensão n, então $H_{n-1}(K)$ possui um subgrupo isomorfo a $\mathbb{Z}_{2}$.

Demonstração 2.3.1.1 Ver. Lima (2012)

\subsubsection{Classe Fundamental:}

Definição 2.3.5 Sejam K uma pseudo-variedade irredutível, finita, orientada, de dimensão $n e$ $\left\{\sigma_{1}, \ldots, \sigma_{k}\right\}$ os $n$-simplexos de $K$ orientados coerentemente com $[K]=\sigma_{1}+\sigma_{2}+\ldots+\sigma_{k} \in C_{n}(K)$. Então, $\partial[K]=0$ (cada face aparece duas vezes com sinal trocado) segue que $[K] \in H_{n}(K)$ é a classe fundamental de $K$.

Teorema 2.3.2 Seja K uma pseudo-variedade fechada, irredutível, de dimensão n. Então:

(1) Se $K$ é orientável $\Rightarrow H_{n}(K)=\mathbb{Z}$.

(2) Se $K$ não é orientável $\Rightarrow H_{n}(K)=\{0\}$.

\section{Demonstração 2.3.2.1}

(1) Note que $C_{n+1}(K)=\{0\}$ (dim. $\left.K=n\right) \Rightarrow H_{n}(K)=Z_{n}(K)\left([z]=\left[z^{\prime}\right] \Longleftrightarrow z=z^{\prime}\right)$

Seja $z \in Z_{n}(K)$ um n-ciclo, então, $z=a_{1} \sigma_{1}+a_{2} \sigma_{2}+\ldots+a_{k} \sigma_{k}$ como $\partial(z)=0$ segue que: $\sum_{i j}\left(a_{i}-a_{j}\right) \tau_{i j}=0 \Longleftrightarrow a_{i}=a_{j}=a, \forall i, j$ portanto $z=a\left(\sigma_{1}+\sigma_{2}+\ldots+\sigma_{k}\right)=a[K]$.

(2) Suponha que $0 \neq z \in Z_{n}(K)$ é um n-ciclo então $z=a_{1} \sigma_{1}+\ldots+a_{n} \sigma_{n}$. Trocando as orientações de $\sigma_{i}$ (se necessário), podemos supor que $a_{i} \geq 0, \forall i$, então, $0=\partial z= \pm\left(a_{i}-a_{j}\right) \tau_{i j}+$ outros $(n-1)$ - simplexos $a_{i}=a_{j}, \forall i, j \Rightarrow a_{i}>0, \forall i, j \Rightarrow 0=\partial z=\partial\left(a \sigma_{1}+a \sigma_{2}+\ldots+a \sigma_{n}\right)=$ $a \sum_{i, j}\left(\tau_{i j}+\tau_{j i}\right) \Rightarrow \tau_{i j}=-\tau_{j i} \Rightarrow K$ é orientável.

\subsubsection{Pseudo - Variedades com Bordo:}

Definição 2.3.6 Uma pseudo-variedade irredutivel de dimensão $n$ com bordo é um complexo simplicial $K$, homogêneo e conexo por $n$-cadeias, e um subcomplexo $\partial K \subset K$ tal que:

(1) Todo $(n-1)$ - simplexo de $K-\partial K$ é face de exatamente dois $n$-simplexos;

(2) Todo $(n-1)$-simplexo de $\partial K$ é face de exatamente um n-simplexo;

(3) $\partial K$ é uma pseudo-variedade (sem bordo) de dimensão $n-1$. 
Definição 2.3.7 Uma pseudo-variedade $K$ é fechada se $K$ é finito $(\Longleftrightarrow|K|$ é compacto) $e$ $\partial K=\emptyset$.

Teorema 2.3.3 Seja K irredutivel de dimensão n. Se $\partial K \neq \emptyset$, então $H_{n}(K)=\{0\}$.

Demonstração 2.3.3.1 Sejam $\left\{\sigma_{1}, \ldots, \sigma_{k}\right\}$ n-simplexos de $K$ e $\left\{\tau_{1}, \ldots, \tau_{l}\right\}(n-1)$-simplexos de $\partial K$.

Se $\partial\left(a_{1} \sigma_{1}+\ldots+a_{k} \sigma_{k}\right)=0 \Rightarrow a_{1} \tau_{1}+\ldots+a_{l} \tau_{l}=0 \Rightarrow a_{1}$ (se $l=k$ acabou, caso contrário $) \Rightarrow z=$ $a_{l+1} \sigma_{l+1}+\ldots+a_{k} \sigma_{k}$ existe $i \in\{l+1, \ldots, k\}, j \in\{1, \ldots, l\}$ tal que $\sigma_{i} \cap \sigma_{j}$ é um $(n-1)$-simplexo de $K, \partial z=0 \Rightarrow a_{i}=0$ (assuma $\left.i=l+1\right) \Rightarrow z=a_{l+2} \sigma_{l+2}+\ldots+a_{k} \sigma_{k}$.

\subsection{Homologia Singular}

\subsubsection{Homologia Singular de $X$ com coeficientes em um anel $R$ :}

Definição 2.4.1 (Simplexo Singular) Seja X um espaço topológico. Um p-simplexo singular em $X$ é uma aplicação contínua $\sigma: \Delta^{p} \rightarrow X$.

Definição 2.4.2 (Aplicação de Faces) (Bredon (1993))

$O(p-1)$-simplexo singular $F_{i}: \Delta^{p-1} \rightarrow \Delta^{p}$ é chamado $i$-ésima aplicação de faces, onde $F_{i}=$ $\left[e_{0}, \ldots, \widehat{e}_{i}, \ldots, e_{p}\right]$.

Definição 2.4.3 O complexo de cadeias singulares de $X$ com coeficientes em $R$ (anel com unidade) é o complexo de p-cadeias $\left(C_{p}(X, R), \partial\right)$ onde

$$
\begin{aligned}
C_{p}(X, R)= & \left\{\sum_{\sigma} r_{\sigma} \sigma(\text { finita }) \mid \sigma: \Delta^{p} \rightarrow X\right\} \text { e } \partial: C_{p}(X, R) \rightarrow C_{p-1}(X, R) ; \partial \sigma=\sum_{i=0}^{p}(-1)^{i} \sigma \circ F_{i} \\
& \ldots \stackrel{\partial_{p+2}}{\longrightarrow} C_{p+1}(X, R) \stackrel{\partial_{p+1}}{\longrightarrow} C_{p}(X, R) \stackrel{\partial_{p}}{\longrightarrow} C_{p-1}(X, R) \rightarrow \ldots \stackrel{\partial_{1}}{\longrightarrow} C_{0}(X, R) \stackrel{\partial_{0}}{\longrightarrow} 0
\end{aligned}
$$

Notação: $C_{p}(X, R)=C_{p}^{\text {Sing }}(X, R):=$ complexo de $p$-cadeias singulares com coeficientes em $R$.

As definições a seguir, constituem definições generalizadas daquelas apresentadas em homologia simplicial.

Proposição 2.4.1 $\partial \circ \partial=\partial^{2}=0$.

Demonstração 2.4.1.1

$$
\begin{aligned}
\partial \partial \sigma & =\partial\left(\sum_{i}(-1)^{i} \sigma \circ F_{i}\right) \\
& =\sum_{i}(-1)^{i} \sum_{j}(-1)^{j} \sigma \circ F_{i} \circ F_{j} \\
& =\sum_{i, j}(-1)^{i+j} \sigma \circ F_{i} \circ F_{j} \\
& =\sum_{i \leq j}\left[(-1)^{i+j} \sigma \circ F_{i} \circ F_{j}\right]+\sum_{j<i}\left[(-1)^{i+j} \sigma \circ F_{j} \circ F_{i-1}\right] \\
& =0 .
\end{aligned}
$$

Definição 2.4.4 Definimos a homologia singular de um espaço topológico $X$ com coeficientes em $R$ como sendo o quociente: 


$$
H_{p}(X, R)=H_{p}^{\operatorname{Sing}}(X, R)=\frac{Z_{p}(X, R)}{B_{p}(X, R)}
$$

onde

$$
Z_{p}(X, R)=K \operatorname{Ker}\left\{\partial: C_{p}(X, R) \rightarrow C_{p-1}(X, R)\right\}
$$

é o conjunto dos p-ciclos singulares em $C_{p}(X, R)$ e,

$$
B_{p}(X, R)=\operatorname{Im}\left\{\partial: C_{p+1}(X, R) \rightarrow C_{p}(X, R)\right\}
$$

é o conjunto Imagem formado pelos p-bordos singulares em $C_{p}(X, R)$.

Definição 2.4.5 (Aplicação Induzida) Seja $f: X \rightarrow Y$ uma aplicação contínua entre espaços topológicos. A função

$$
\begin{gathered}
f_{\#}: C_{p}(X, R) \rightarrow C_{p}(Y, R) \\
f_{\#}(\sigma)=f \circ \sigma
\end{gathered}
$$

é chamada aplicacão de cadeias induzida pela $f$.

Proposição 2.4.2 A aplicação induzida $f_{\#}$ é uma aplicação de cadeia, isto é,

$$
f_{\#} \circ \partial=\partial \circ f_{\#}
$$

Demonstração 2.4.2.1 $f_{\#} \partial(\sigma)=f_{\#}\left(\sum_{i}(-1)^{i} \sigma \circ F_{i}\right)=\sum_{i}(-1)^{i}\left(f \circ \sigma \circ F_{i}\right)=\partial f_{\#}(\sigma)$.

Para cada p, o quadrado no diagrama abaixo é comutativo.

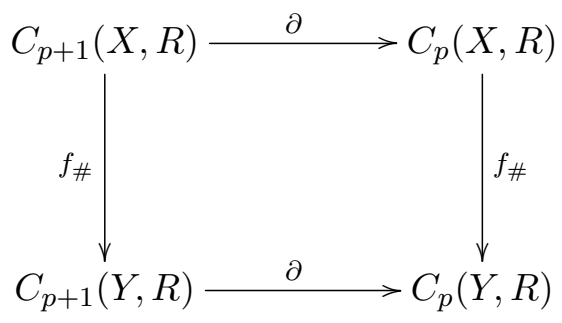

Essa aplicação induzida pela $f$, por sua vez, induz uma aplicação nos grupos de homologia singular dos respectivos complexos de cadeias associados por ela.

Definição 2.4.6 Seja $f_{\#}$ uma aplicação de cadeia induzida por uma $f: X \rightarrow Y$ contínua onde $X$ e Y são espaços topológicos. A função

$$
\begin{gathered}
f_{*}: H_{p}(X, R) \rightarrow H_{p}(Y, R) \\
f_{*}[\sigma]=\left[f_{*} \sigma\right]
\end{gathered}
$$

é a função induzida pela aplicação de cadeia $f_{\#}$ nos grupos de homologia singular.

Proposição 2.4.3 Se $X=\amalg_{\alpha \in \Lambda} X_{\alpha}$ (com topologia da união disjunta onde $X_{\alpha} \subset X$ são abertos) então, $H_{p}(X, R)=\bigoplus_{\alpha \in \Lambda} H_{p}\left(X_{\alpha}, R\right), \quad \forall p$.

Demonstração 2.4.3.1 Se $C \subset X$ é uma componente conexa por arcos, então $C \subset X_{\alpha}$ para algum $\alpha \in \Lambda$. Como $\Delta^{p}$ é conexo por arcos e $\sigma: \Delta^{p} \rightarrow X$ é contínua, então $\operatorname{Im} \sigma \subset X_{\alpha}$ para um único $\alpha \in \Lambda, \log o$

$$
\left.C_{p}(X, R)=\bigoplus_{\alpha \in \Lambda} C_{p}\left(X_{\alpha}, R\right) \quad \& \quad \partial: C_{p}\left(X_{\alpha}, R\right)\right) \rightarrow C_{p-1}\left(X_{\alpha}, R\right)
$$

e, portanto,

$$
H_{p}(X, R)=\bigoplus_{\alpha \in \Lambda} H_{p}\left(X_{\alpha}, R\right)
$$


Proposição 2.4.4 Se $X$ é um espaço topológio formado por apenas um ponto, isto é, $X=\left\{x_{0}\right\}$, então

$$
H_{p}(X, R)=\left\{\begin{array}{cc}
R & \text { se } p=0 \\
0 & \text { se } p>0
\end{array}\right.
$$

Demonstração 2.4.4.1 Para cada $p$, existe um único p-simplexo singular $\sigma_{p}: \Delta^{p} \rightarrow\left\{x_{0}\right\}$ (aplicação constante), desta forma, teremos o complexo de cadeias singulares $\left(C_{p}(X, R), \partial\right)$ como sendo

$$
\ldots R \stackrel{\partial_{p+1}}{\longrightarrow} R \stackrel{\partial_{p}}{\longrightarrow} R \stackrel{\partial_{p-1}}{\longrightarrow} \ldots \stackrel{\partial_{1}}{\longrightarrow} R \stackrel{\partial_{0}}{\longrightarrow} 0
$$

Onde em grau $p$, tem-se $R \cong\left\langle\sigma_{p}\right\rangle$. Ainda para cada $p$, teremos $\partial_{p}$ calculado como segue:

$$
\partial_{p}\left(\sigma_{p}\right)=\sum_{i=0}^{p}(-1)^{i} F_{i} \circ \sigma_{p}=\sum_{i=0}^{p}(-1)^{i} \sigma_{p-1}=\left\{\begin{array}{cc}
\sigma_{p-1} & \text { se p é par } \\
0 & \text { se p é impar. }
\end{array}\right.
$$

Com os resultados anteriores, teremos o complexo de cadeias singulares reescrito como,

$$
\ldots R \stackrel{\mathbb{1}_{R}}{\rightarrow} R \stackrel{0}{\rightarrow} R \stackrel{\mathbb{1}_{R}}{\longrightarrow} \ldots R \stackrel{0}{\rightarrow} R \stackrel{\mathbb{1}_{R}}{\rightarrow} 0
$$

Portanto,

$$
H_{p}\left(\left\{x_{0}\right\}, R\right)=\left\{\begin{array}{cc}
R & \text { se } p=0 \\
\frac{R}{R}=0 & \text { se p é ímpar } \\
\frac{0}{0}=0 & \text { se p é par }
\end{array}\right.
$$

\subsubsection{Homotopia e Equivalência Homotópica:}

Antes de passarmos aos exemplos propriamente ditos do cálculo dos grupos de homologia singular, apresentaremos um conceito importante e que nos ajudará neste objetivo.

Definição 2.4.7 (Homotopia) Sejam $f, g: X \rightarrow Y$ funções contínuas entre espaços topológicos, $X$ e $Y$. Uma homotopia entre $f, g: X \rightarrow Y$ é uma função contínua

$$
\begin{gathered}
H: X \times I \longrightarrow Y \\
H(x, 0)=f(x) \quad \text { e } H(x, 1)=g(x), \quad \forall x \in X .
\end{gathered}
$$

Neste caso, escreveremos $f \sim g$ ( $f$ é homotópico à $g$ ).

Proposição 2.4.5 é uma relação de equivalência em $\mathcal{C}(X, Y)$.

Demonstração 2.4.5.1 Sejam $f, g, h \in \mathcal{C}(X, Y)$, então:

(1) Temos que $f \sim f$, para $H(x, t)=f(x), \forall t \in I$.

(2) Se $f \sim g$ existe $H(x, t)$ homotopia entre $f$ e $g \Longrightarrow$ fazendo $\bar{H}(x, t)=H(x, 1-t)$ teremos $g \sim f$.

(3) Sejam $H: f \sim g$ e $G: g \sim h$, definimos $H * G: f \sim h$ como sendo

$$
H * G(x, t)=\left\{\begin{array}{ccc}
H(x, 2 t) & \text { se } & 0 \leq t \leq \frac{1}{2} \\
G(x, 2 t-1) & \text { se } & \frac{1}{2} \leq t \leq 1
\end{array}\right.
$$

então $H * G: f \sim h$. 
Segue que $\sim$ é uma relação de equivalência em $\mathcal{C}(X, Y)$.

Exemplo 2.4.1 (Homotopia Linear) Seja $\mathbb{E}$ um espaço vetorial, $Y \subset \mathbb{E}$ subespaço. Se $f, g$ : $X \rightarrow Y$ continuas, tais que o segmento de reta $\overline{f(x) g(x)} \subset Y \Rightarrow f \sim g$ através da homotopia (linear), $H(x, t)=(1-t) f(x)+t g(x)$.

Definição 2.4.8 (Homotopia Relativa) Seja $A \subset X, X$ espaço topológico. Dadas $f, g: X \rightarrow Y$, continuas, diremos que $f$ é homotópica à $g$ relativa ao conjunto $A$, escrevendo $f \sim g($ rel $A)$ se existir $H: X \times I \rightarrow Y$ tal que:

$$
\begin{array}{cc}
H(x, 0)=f(x), & \forall x \in X \\
H(x, 1)=g(x), & \forall x \in X \\
H(x, t)=a, & \forall a \in A
\end{array}
$$

Definição 2.4.9 (Equivalência de Homotopia) Uma $f: X \rightarrow Y$ contínua será chamada equivalência de homotopia quando existir $g: Y \rightarrow X$, tal que:

$$
\left\{\begin{array}{l}
f \circ g \sim \mathbb{1}_{Y} \\
g \circ f \sim \mathbb{1}_{X} .
\end{array}\right.
$$

Neste caso, os espaços topológicos $X$ e $Y$ serão homotopicamente equivalentes (mesmo tipo de homotopia), cuja notação será, $X \sim Y$.

Definição 2.4.10 Diremos que um espaço topológico é contrátil quando for homotopicamente equivalente ao espaço formado por apenas um ponto, isto é,

$$
X \text { é contrátil } \Longleftrightarrow X \sim\{*\} .
$$

\section{Exemplo 2.4.2}

(1) Todo espaço vetorial $\mathbb{E}$ é contrátil. Basta considerar a aplicação:

$$
\begin{gathered}
H(x, t): \mathbb{E} \times I \rightarrow \mathbb{E} \\
H(x, t)=t x
\end{gathered}
$$

(2) A aplicação $i: \mathbb{S}^{n-1} \rightarrow \mathbb{R}^{n}-\{\mathbf{0}\}$ é equivalência de homotopia.

Consideramos

$$
\begin{aligned}
& r: \mathbb{R}^{n}-\{\mathbf{0}\} \underset{x}{ } \rightarrow \mathbb{S}^{n-1} \\
& x \mapsto \frac{x}{\|x\|} \\
& H: \mathbb{R}^{n}-\{\mathbf{0}\} \times I \rightarrow \mathbb{R}^{n}-\{\mathbf{0}\} \\
& H(x, t)=t x+(1-t) \frac{x}{\|x\|}
\end{aligned}
$$

Um caso particular de equivalência de homotopia importante é o de retrato por deformação.

Definição 2.4.11 (Retração) Seja $A \subset X, X$ espaço topológico. Uma retração de $X$ em $A$ é uma aplicação $r: X \rightarrow A$ tal que $r \circ i=\mathbb{1}_{A}\left(\left.r\right|_{A}=\mathbb{1}_{A}\right)$.

Definição 2.4.12 (Retrato por deformação) Uma retração é um retrato por deformação se:

$$
i \circ r \sim \mathbb{1}_{X}(\operatorname{rel} A) .
$$




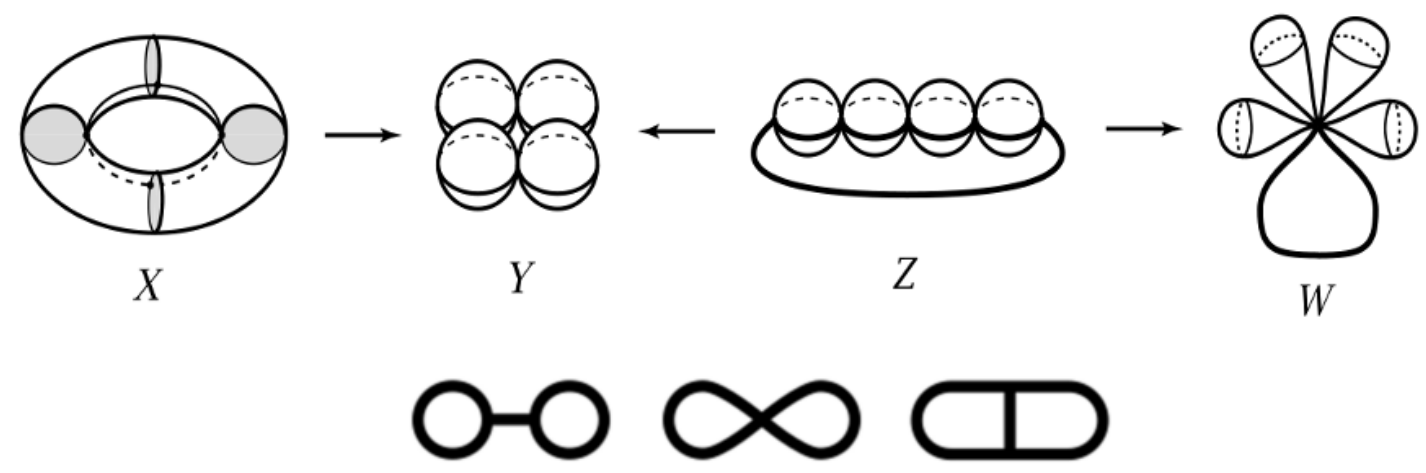

Figura 2.17: Espaços Homotopicamente Equivalentes (Hatcher (2001)).

Teorema 2.4.1 (Invariância de Homotopia) Se $f, g: X \rightarrow Y$ são contínuas e homotópicas então, as aplicações induzidas respectivamente por $f$ e $g$, a saber, $f_{*}$ e $g_{*}$ coincidem como aplicações dos grupos de homologia das cadeias singulares geradas por $X$ e $Y$, isto é, $f_{*}=g_{*}: H_{p}(X, R) \rightarrow$ $H_{p}(Y, R)$.

Demonstração 2.4.1.1 Por hipótese, existe uma $H: X \times I \rightarrow Y$, homotopia entre $f$ e g. De posse desta informação, iremos construir, com auxílio de $H$, uma nova homotopia $h$, agora de cadeia, entre as aplicações $f_{*}$ e $g_{*}$ ou seja,

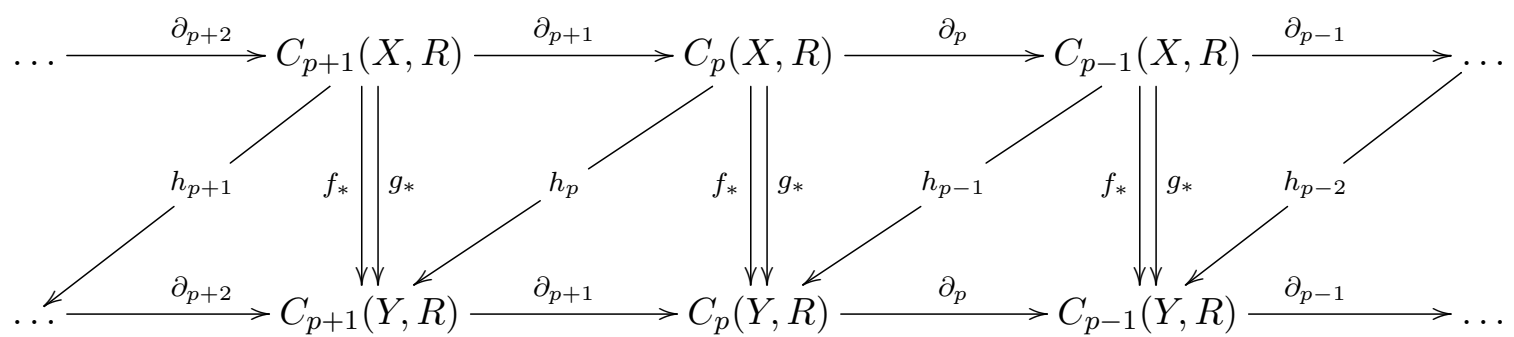

onde $g_{*}-f_{*}=\partial h+h \partial$, para $h$ e $\partial$ adequados na cadeia.

Como $\Delta^{p}$ não é um $(p+1)$-simplexo, poderemos decompô-lo de forma natural com sendo uma "soma" de $(p+1)$. Por exemplo, $\Delta^{1} \times I \cong I \times I$

Em geral, considerando em $\Delta^{p} \times I, v_{i}\left(e_{i}, 0\right), w_{i}=\left(e_{i}, 1\right)$, teremos as inclusões:

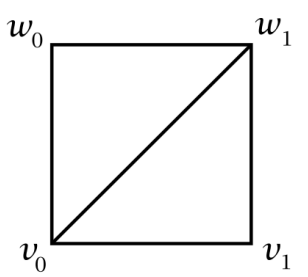

Figura 2.18: $\Delta^{1} \times I \cong I \times I$

$$
\begin{gathered}
L_{i}: \Delta_{p+1} \rightarrow \Delta \times I \\
L_{i}\left(e_{0}, \ldots, e_{p}\right)=\left(v_{0}, \ldots, v_{i}, w_{i}, w_{i+1}, \ldots, w_{p}\right)
\end{gathered}
$$

Obteremos assim, para cada $\sigma: \Delta^{p} \rightarrow X$,

$$
\Delta^{p+1} \stackrel{L_{i}}{\longrightarrow} \Delta^{p} \times I \stackrel{\sigma \times \mathbb{1}}{\longrightarrow} X \times I \stackrel{H}{\longrightarrow} Y
$$


Definimos então,

$$
\begin{gathered}
h_{p}: C_{p}(X, R) \rightarrow C_{p+1}(Y, R) \\
h_{p}(\sigma)=\sum_{i=0}^{p}(-1)^{i} H \circ(\sigma \times \mathbb{1}) \circ L_{i}
\end{gathered}
$$

Temos que $\partial h+h \partial=g_{*}-f_{*}$. De fato, pois:

$$
\begin{aligned}
\partial h_{p}(\sigma)\left(e_{0}, \ldots, e_{p}\right) & =\sum_{j=0}^{p+1}(-1)^{j} h_{p}(\sigma) \circ F_{j}\left(e_{0}, \ldots, e_{p}\right) \\
& =\sum_{j \leq i}(-1)^{j}(-1)^{i} H \circ(\sigma \times \mathbb{1})\left(v_{0}, \ldots, \widehat{v}_{j}, \ldots, v_{i}, w_{i}, \ldots, w_{p}\right) \\
& +\sum_{j \geq i}(-1)^{j+1}(-1)^{i} H \circ(\sigma \times \mathbb{1})\left(v_{0}, \ldots, v_{i}, w_{i}, \ldots, \ldots, \widehat{w_{j}}, \ldots, w_{p}\right)
\end{aligned}
$$

$e$

$$
\begin{aligned}
h_{p-1}(\partial \sigma)\left(e_{0}, \ldots, e_{p}\right) & =h_{p-1}\left(\sum_{j=0}^{p}(-1)^{j} \sigma \circ F_{j}\right)\left(e_{0}, \ldots, e_{p}\right) \\
& =\sum_{j<i}(-1)^{i-1}(-1)^{j} H \circ(\sigma \times \mathbb{1})\left(v_{0}, \ldots, \widehat{v}_{j}, \ldots, v_{i}, w_{i}, \ldots, w_{j}, \ldots, w_{p}\right) \\
& +\sum_{j>i}(-1)^{i}(-1)^{j} H \circ(\sigma \times \mathbb{1})\left(v_{0}, \ldots, v_{i}, w_{i}, \ldots, \widehat{w_{j}}, \ldots, w_{p}\right) .
\end{aligned}
$$

$\log$,

$$
\begin{aligned}
(\partial h+h \partial)(\sigma) & =H \circ(\sigma \times \mathbb{1})\left(\widehat{v_{0}}, w_{0}, \ldots, w_{p}\right)-H \circ(\sigma \times \mathbb{1})\left(v_{0}, \ldots, v_{p}, \widehat{w_{p}}\right) \\
& =g \circ \sigma-f \circ \sigma \\
& =g_{*}(\sigma)-f_{*}(\sigma) \square .
\end{aligned}
$$

Corolário 2.4.1.1 Se $f: X \rightarrow Y$ é uma equivalência de homotopia, então $f_{*}: H_{p}(X, R) \rightarrow$ $H_{p}(Y, R)$ é um isomorfismo para todo $p$.

Demonstração 2.4.1.1.1 Como $f$ é equivalência de homotopia, existe uma $g: Y \rightarrow X$ (inversa homotópica de $f$ ), tal que:

$$
\left\{\begin{array}{l}
f \circ g \sim \mathbb{1}_{Y} \\
g \circ f \sim \mathbb{1}_{X}
\end{array}\right.
$$

\subsubsection{Sequências Exatas}

Definição 2.4.13 (Sequências Exatas) Sejam $\mathcal{A}=\left(A_{n}, \partial_{A_{n}}\right), \mathcal{B}=\left(B_{n}, \partial_{B_{n}}\right)$ e $\mathcal{C}=\left(C_{n}, \partial_{C_{n}}\right)$ complexos de cadeias singulares. Uma sequência exata curta de complexos é uma sequência

$$
0 \longrightarrow \mathcal{A} \stackrel{\phi}{\rightarrow} \mathcal{B} \stackrel{\psi}{\rightarrow} \mathcal{C} \longrightarrow 0
$$

de aplicações de cadeia $\phi$ e $\psi$ tal que a sequência,

$$
0 \longrightarrow A_{n} \stackrel{\phi_{n}}{\longrightarrow} B_{n} \stackrel{\psi_{n}}{\longrightarrow} C_{n} \longrightarrow 0
$$

é exata $\forall n$, ou seja, 
(1) $\operatorname{Im}\left(\phi_{n}\right)=\operatorname{Ker}\left(\psi_{n}\right)$;

(2) $\phi_{n}$ é injetor $\left(\operatorname{Ker}\left(\phi_{n}\right)=0=\operatorname{Im}\left(0 \hookrightarrow A_{n}\right)\right)$;

(3) $\psi_{n}$ é sobrejetor $\left(\operatorname{Im}\left(\phi_{n}\right)=\operatorname{Ker}\left(C_{n} \hookrightarrow\{0\}\right)\right)$.

Teorema 2.4.2 Dada uma sequência curta exata

$$
0 \longrightarrow \mathcal{A} \stackrel{\phi}{\rightarrow} \mathcal{B} \stackrel{\psi}{\rightarrow} \mathcal{C} \longrightarrow 0
$$

existe $\forall n \in \mathbb{N}$ um morfismo

$$
\partial_{*}: H_{n}(\mathcal{C}) \rightarrow H_{n-1}(\mathcal{A})
$$

tal que a sequência

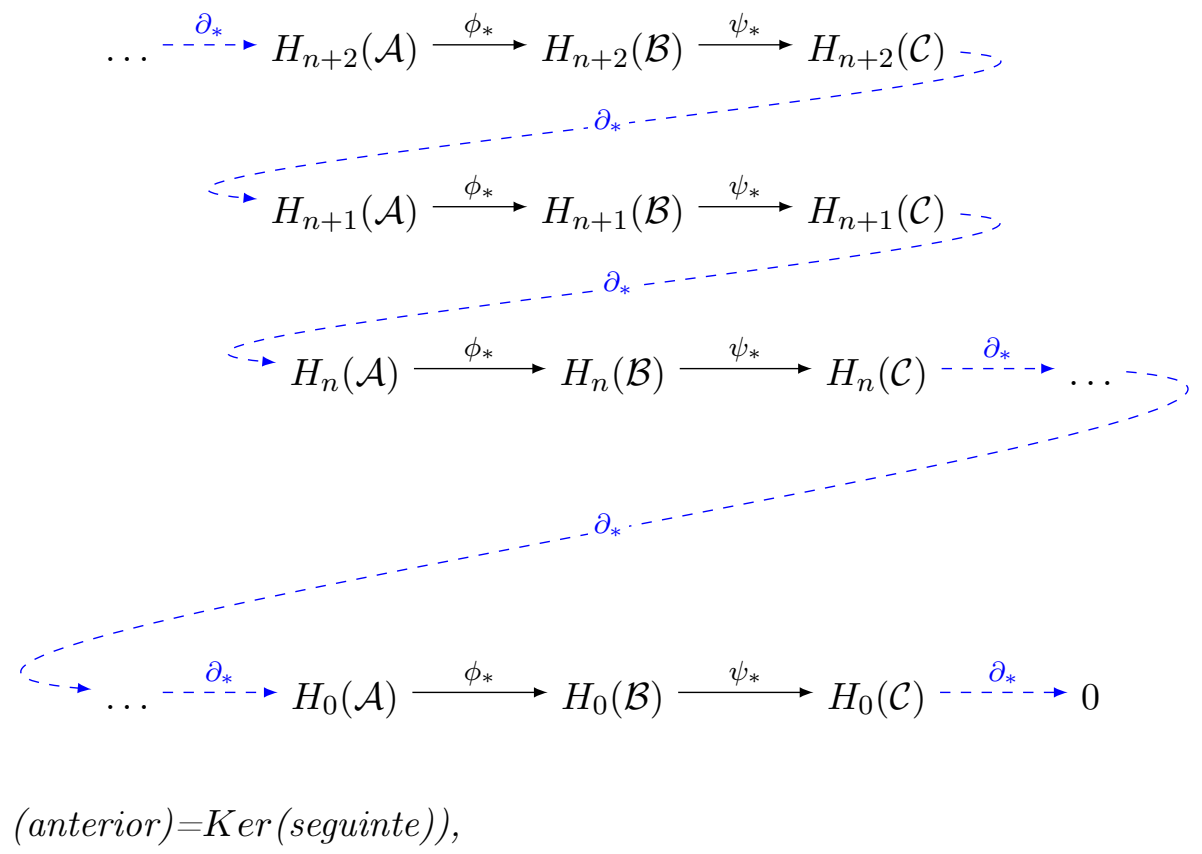

Demonstração 2.4.2.1 (Construção de $\partial_{*}$ ) Como se trata de um teorema de existência, iremos construir a aplicação $\partial_{*}$. 


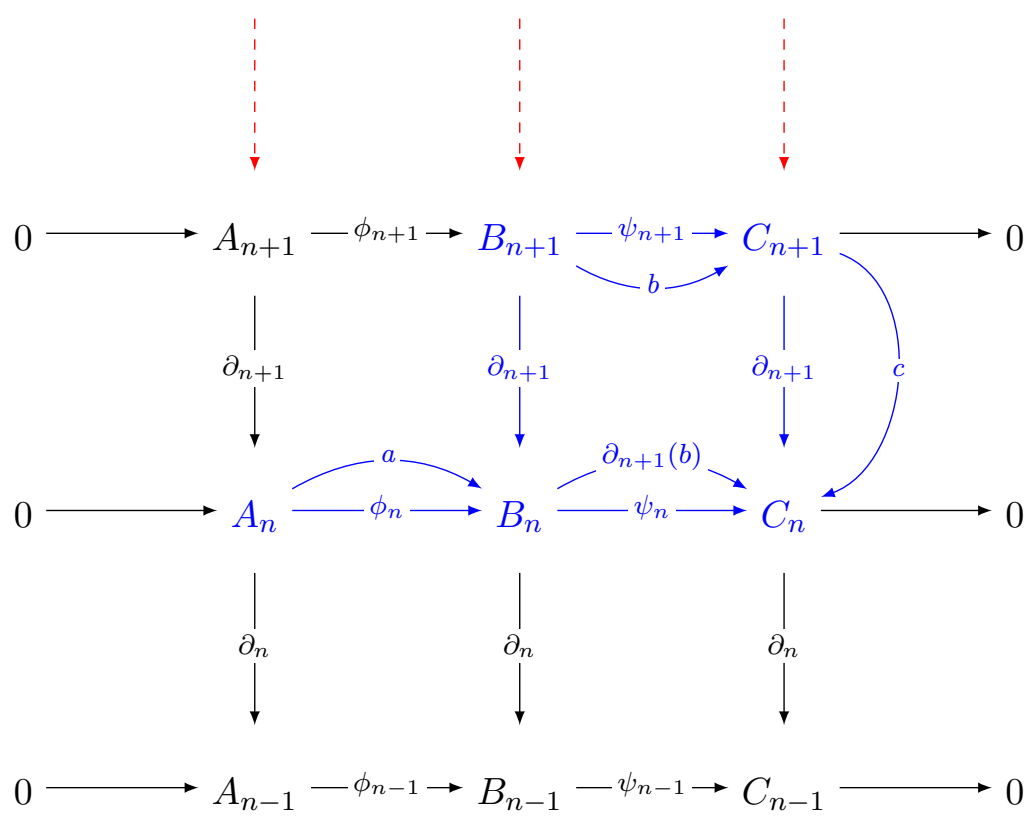

Seja $c \in C_{n+1}$ um ciclo, isto é, $\partial_{n+1}(c)=0$. Como existe $b \in B_{n+1}$ tal que $\psi_{n+1}(b)=c$, segue pela definição de $\psi_{n+1}\left(\partial_{n+1} \circ \psi_{n+1}=\psi_{n} \circ \partial_{n+1}\right)$ que:

$$
\begin{aligned}
\psi_{n}\left(\partial_{n+1}(b)\right) & =\left(\psi_{n} \circ \partial_{n+1}\right)(b) \\
& =\left(\partial_{n+1} \circ \psi_{n+1}\right)(b) \\
& =\partial_{n+1}\left(\psi_{n+1}(b)\right) \\
& =\partial_{n+1}(c) \\
& =0 .
\end{aligned}
$$

Portanto, $\partial_{n+1}(b) \in \operatorname{Ker}\left(\psi_{n}\right)=\operatorname{Im}\left(\phi_{n}\right) \Rightarrow \exists a \in A_{n} ; \phi_{n}(a)=\partial_{n+1}(b)$

(1) $\partial_{n+1}(a)=0\left(\llbracket a \rrbracket \in H_{n}(\mathcal{A})\right), \phi_{n}\left(\partial_{n+1}\right)(a)=\partial(\phi(a))=0$ ( $\phi$ é injetor $)$

(2) $\partial_{*}$ não depende da escolha de $\left.b \mapsto c: \psi\left(b^{\prime}\right)=c \Rightarrow\left(b-b^{\prime}\right)=0 \Rightarrow b-b^{\prime}=\phi \bar{a}\right) \Rightarrow \partial(\bar{a})=a-a^{\prime}$ pois $\phi(\partial \bar{a})=\partial(\phi(\bar{a}))=\partial b-\partial \bar{a}=\phi(a)-\phi\left(a^{\prime}\right)$

(3) $\partial \llbracket c \rrbracket n \tilde{a} o$ depende do representante c: Suponha que $c=\partial \bar{a}$ e seja $\bar{b}$ tal que $\psi(\bar{a})=\bar{c} \Rightarrow \psi(\partial \bar{b}-$ $b)=\partial(\psi \bar{b})-\psi(b)=0 \Rightarrow \psi(\partial \bar{b})=\psi(b)=c \Rightarrow \llbracket a \rrbracket=\llbracket a^{\prime} \rrbracket$ onde $\phi\left(a^{\prime}\right)=\partial \partial \bar{b}=0 \Rightarrow a^{\prime}=0$

(4) Exato em $H_{n}(\mathcal{A})$ (isto é, $\operatorname{Im}\left(\partial_{*}\right)=\operatorname{Ker}\left(\phi_{*}\right)$ ):

(i) $\operatorname{Im}\left(\partial_{*}\right) \subset \operatorname{Ker}\left(\phi_{*}\right)\left(\phi_{*} \circ \partial_{*}=0\right)$

$$
\phi_{*} \partial_{*}(\llbracket c \rrbracket)=\llbracket \phi(a) \rrbracket=\llbracket \partial b \rrbracket=0 .
$$


(ii) $\operatorname{Ker}\left(\phi_{*}\right) \subset \operatorname{Im}\left(\partial_{*}\right)$ Suponha $\phi_{*}(\llbracket a \rrbracket)=\llbracket \phi(a) \rrbracket=0 \Rightarrow \phi(a)=\partial b$. Seja $c=\psi(\partial b)$, então $\partial(c)=\partial \psi(\partial b)=\partial^{2} \psi(b)=0 \Rightarrow \llbracket c \rrbracket \in H_{n-1}(\mathcal{C})$. Mas $\partial_{*}(\llbracket c \rrbracket)=\llbracket a \rrbracket$. (Por construção)

(5) Exato em $H_{n}(\mathcal{C}):\left(\operatorname{Ker}\left(\partial_{*}\right)=\operatorname{Im}\left(\psi_{*}\right)\right.$

(i) $\operatorname{Im}\left(\psi_{*}\right) \subset \operatorname{Ker}\left(\partial_{*}\right):$

$$
\partial_{*} \phi_{*}(\llbracket b \rrbracket)=\partial_{*}(\llbracket \phi(b) \rrbracket)=\llbracket a \rrbracket ; \phi(a)=\psi(b)=0 .
$$

(ii) $\operatorname{Ker}\left(\partial_{*}\right) \subset \operatorname{Im}\left(\psi_{*}\right)$, suponha que $\partial_{*}(\llbracket c \rrbracket)=\llbracket a \rrbracket=0 \Rightarrow \bar{a} \in C_{n}(\mathcal{A}), \partial \bar{a}=a \Rightarrow \partial \phi(\bar{a})=$ $\phi(a)=\partial(b) \Rightarrow \partial(b-\phi \bar{a})=0 \Rightarrow \llbracket b-\phi(\bar{a}) \rrbracket \in H_{n}(\mathcal{B}) \operatorname{mas} \psi(b-\phi(\bar{a})=\psi(b)-\psi(\phi(\bar{a}))=$ $\psi(b)=c$.

Proposição 2.4.6 (Sequência de Mayer-Vietoris) Seja $K$ um complexo simplicial e sejam $K_{1}$ e $K_{2}$ subcomplexos tais que $K=K_{1} \cup K_{2}$. Então a sequência

$$
0 \longrightarrow C_{p}\left(K_{1} \cap K_{2}\right) \stackrel{i_{\#}}{\longrightarrow} C_{p}\left(K_{1}\right) \bigoplus C_{p}\left(K_{2}\right) \stackrel{j_{\#}}{\longrightarrow} C_{p}\left(K_{1} \cup K_{2}\right) \longrightarrow 0
$$

é exata. Observamos que as inclusões naturais $i_{\alpha}: K_{1} \cap K_{2} \rightarrow K_{\alpha}$ e $j_{\alpha}: K_{\alpha} \rightarrow K_{1} \cup K_{2}$ para $\alpha: 1,2$ induzem, ao nivel das cadeias, os homomorfismos:

$$
i_{\#}: C_{p}\left(K_{1} \cap K_{2}\right) \rightarrow C_{p}\left(K_{1}\right) \bigoplus C_{p}\left(K_{2}\right) \text { tal que } c \mapsto\left(i_{1_{\#}}(c), i_{2_{\#}}(c)\right)
$$

$e$

$$
\left.j_{\#}: C_{p}\left(K_{1}\right) \bigoplus C_{p}\left(K_{2}\right) \rightarrow C_{p}\left(K_{1} \cup K_{2}\right) \text { tal que }\left(c, c^{\prime}\right) \mapsto j_{1_{\#}}(c)-j_{2_{\#}}\left(c^{\prime}\right)\right)
$$

Obtemos assim uma sequência longa exata (sequência de Mayer-Vietoris)

$$
\begin{array}{r}
\ldots \partial_{*}-H_{n+2}\left(K_{1} \cap K_{2}\right) \stackrel{i_{*}}{\longrightarrow} H_{n+2}\left(K_{1}\right) \oplus H_{n+2}\left(K_{2}\right) \stackrel{j_{*}}{\longrightarrow} H_{n+2}\left(K_{1} \cup K_{2}\right) \ldots=\text {, } \\
\quad \therefore H_{n+1}\left(K_{1} \cap K_{2}\right) \stackrel{i_{*}}{\longrightarrow} H_{n+1}\left(K_{1}\right) \bigoplus H_{n+1}\left(K_{2}\right) \stackrel{j_{*}}{\longrightarrow} H_{n+1}\left(K_{1} \cup K_{2}\right) \_ \text {, }
\end{array}
$$

$$
H_{n}\left(K_{1} \cap K_{2}\right) \stackrel{i_{*}}{\longrightarrow} H_{n}\left(K_{1}\right) \oplus H_{n+1}\left(K_{2}\right) \stackrel{j_{*}}{\longrightarrow} H_{n}\left(K_{1} \cup K_{2}\right) \stackrel{\partial_{*}}{\longrightarrow} \ldots \ldots
$$

$$
\stackrel{\partial_{*}}{\longrightarrow} H_{0}\left(K_{1} \cap K_{2}\right) \stackrel{i_{*}}{\longrightarrow} H_{0}\left(K_{1}\right) \bigoplus H_{0}\left(K_{2}\right) \stackrel{j_{*}}{\longrightarrow} H_{0}\left(K_{1} \cup K_{2}\right) \stackrel{\partial_{*}}{\longrightarrow} 0 .
$$

Demonstração 2.4.6.1 Segue do diagrama apresentado no teorema 2.4.2.

Definição 2.4.14 (Homologia Relativa) Seja $(X, A)$ um par de espaços topológicos (i.e., $A \subset$ $X$ é subspaço de $X)$. O complexo de cadeias singulares formadas nessa par é o quociente relativo aos complexos em separados dos dois espaços:

$$
C_{p}(X, A ; R)=\frac{C_{p}(X, R)}{C_{p}(A, R)}
$$

$\operatorname{com} \partial \bar{\sigma}=\overline{(\partial \sigma)}$ 
Por consequência, sua homologia relativa será o quociente representado por:

$$
H_{p}(X, A ; R)=\frac{H_{p}(X, R)}{H_{p}(A, R)}
$$

Observação 2.4.1 Podemos identificar $0 \neq \bar{\sigma} \in C_{p}(X, A)$ com $\sigma \in C_{p}(X, A)$ tal que $\operatorname{Im}(\sigma) \not \subset A$.

\subsubsection{Sequência Longa do Par}

Uma sequência curta exata

$$
0 \longrightarrow C_{p}(A) \stackrel{i}{\rightarrow} C_{p}(X) \stackrel{j}{\rightarrow} C_{p}(X, A) \longrightarrow 0
$$

Induz a sequência longa exata

$$
\ldots \longrightarrow H_{p}(A) \stackrel{i_{*}}{\rightarrow} H_{p}(X) \stackrel{j_{*}}{\rightarrow} H_{p}(X, A) \stackrel{\partial_{*}}{\rightarrow} H_{p-1}(A) \longrightarrow \ldots
$$

Proposição 2.4.7 (Naturalidade) O morfismo $\partial_{*}: H_{p}(X, A) \rightarrow H_{p-1}(A)$ é natural, isto é, se $f:(X, A) \rightarrow(Y, B)$, então

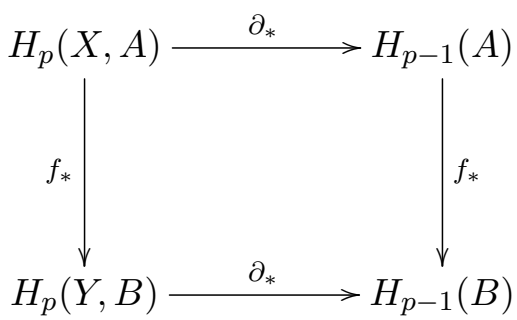

Demonstração 2.4.7.1 $c \in C_{p}(X)$ representa $\llbracket c \rrbracket \in H_{p}(X, A) \Longleftrightarrow \partial c \in C_{p}(A)$.

Segue que

$$
\partial_{*}(\llbracket c \rrbracket)_{H_{p}(X, A)}=\llbracket \partial c \rrbracket_{H_{p-1}(A)}
$$

E portanto,

$$
f_{*}\left(\partial_{*}(\llbracket c \rrbracket)=f_{*}(\llbracket \partial c \rrbracket)_{A}=\llbracket f_{*}(\partial c) \rrbracket_{B}=\llbracket \partial\left(f_{*}(c)\right) \rrbracket_{B}=\partial_{*} f_{*}(\llbracket c \rrbracket)\right.
$$

Corolário 2.4.7.1 Se $A \subset U \subset X$ e $i: A \hookrightarrow U$ é equivalência de homotopia, então $i:(X, A) \hookrightarrow$ $(X, U)$ induz isomorfismo em homologia.

Demonstração 2.4.7.1.1 Temos,

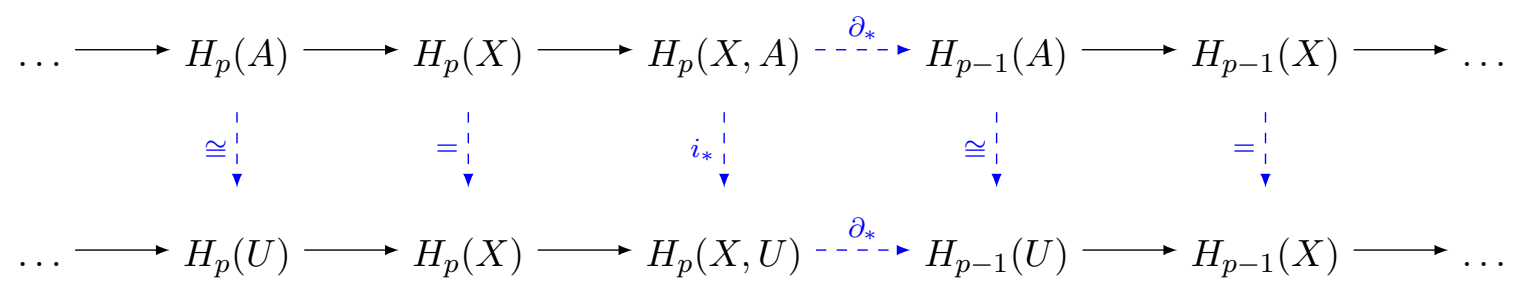

onde cada subdiagrama é comutativo e o resultado segue de:

Lema 2.4.1 (Lema dos Cinco) Seja o diagrama abaixo morfismos entre sequências exatas. 


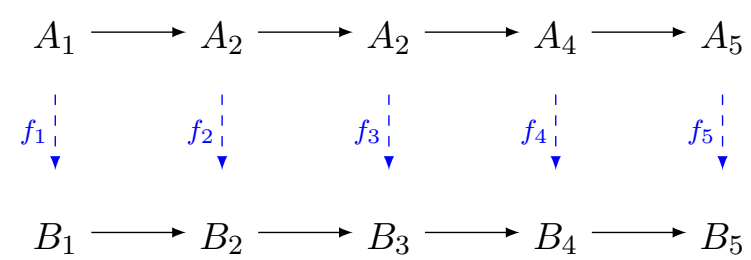

Se $f_{1}, f_{2}, f_{4}$ e $f_{5}$ são isomorfismos então $f_{3}$ é isomorfismo.

Demonstração: Ver Lima (2012), pp. 08.

Definição 2.4.15 (Homologia Reduzida) Considere o complexo aumentado:

$$
\ldots \stackrel{\partial}{\rightarrow}(X, R) \stackrel{\partial}{\rightarrow} C_{p-1}(X, R) \stackrel{\partial}{\rightarrow} \ldots \stackrel{\partial}{\rightarrow} C_{1}(X, R) \rightarrow C_{0}(X, R) \stackrel{\epsilon}{\rightarrow} R \longrightarrow 0
$$

Onde $\epsilon: C_{0}(X, R) \rightarrow R ; \quad \epsilon\left(\sum r_{\sigma} \sigma\right)=\sum r_{\sigma}$. A homologia reduzida de $X$ é a homologia deste complexo (denotada por $\tilde{H}_{p}(X, R)$ ).

\section{Teorema 2.4.3 (Excisão)}

(a) Se $Z \subset A \subset X$ é tal que $\bar{Z} \subset \operatorname{int}(A)$, ( $\bar{Z}$ fecho de $Z$ ) então,

$$
(X-Z, A-Z) \hookrightarrow(X, A) \text { induz um isomorfismo } H_{n}(X-Z, A-Z) \cong H_{n}(X, A), \forall n .
$$

(b) Se $A, B \subset X, X=\operatorname{int}(A) \cup \operatorname{int}(B)$ então,

$$
(B, A \cap B) \hookrightarrow(X, A) \text { induz isomorfismo } H_{n}(B, A \cap B) \cong H_{n}(X, A), \forall n .
$$

Observação 2.4.2 (a) $\Longleftrightarrow(b)$, pois dado $Z \subset A \subset X \operatorname{com} \bar{Z} \subset \operatorname{int}(A)$, tome $B=X-Z$, então

$$
\left\{\begin{array}{l}
\operatorname{int}(A) \cup \operatorname{int}(B)=X \\
A \cap B=A-Z
\end{array}\right.
$$

Dado $A, B$ tome $Z=X-B \subset A$ (Pois int $(A) \cup \operatorname{int}(B)=X)$

Ideia da Excisão: "Homologia é uma soma de efeitos locais" (Efeito em diferença de homotopia)

Formalizando esta ideia: Seja $U=\left\{U_{\alpha}\right\}$ tal que $X=\bigcup_{\alpha} \operatorname{int}\left(U_{\alpha}\right)$

Definição 2.4.16 Um p-simplexo singular $\Delta_{p} \rightarrow X$ é $U$-pequeno se $\operatorname{Im}(\sigma) \subseteq U_{\alpha}$ para algum $\alpha$. Definimos assim o complexo singular formado pelos simplexos singulares U-pequenos, isto é,

$$
C_{p}^{U}(X)=\left\{\sum r_{\sigma} \sigma \mid r_{\sigma} \in R, \sigma e ́ U-\text { pequeno }\right\}
$$

Segue que

$$
\partial: C_{p}^{U}(X) \rightarrow C_{p-1}^{U}(X) \text { e } i: C_{p}^{U} \rightarrow C_{p}(X) \text { é aplicação de cadeias }
$$

Definimos como sendo $H_{p}^{U}(X, R):=$ Homologia deste complexo.

Proposição 2.4.8 Existe $\rho: C_{p}(X) \rightarrow C_{p}^{U}(X)$ tal que $i \circ \rho$ e $\rho \circ i$ são algebricamente homotópicos $\grave{a} \mathbb{1}$, em particular,

$$
H_{p}(X) \cong H_{p}^{U}(X), \forall p
$$


Demonstração 2.4.8.1 Para demonstração ver Hatcher (2001)

Sejam $A, B \subset X$, tal que $X=\operatorname{int}(A) \cup \operatorname{int}(B)$. Então a sequência curta exata:

$$
\begin{gathered}
0 \longrightarrow C_{n}(A \cap B) \stackrel{i}{\longrightarrow} C_{n}(A) \oplus C_{n}(B) \stackrel{s}{\longrightarrow}-C_{n}(A+B) \stackrel{\iota}{\longrightarrow} 0 \\
\sigma(\sigma,-\sigma) \\
(\sigma, \tau) \longrightarrow \sigma+\tau
\end{gathered}
$$

Induz uma sequência longa exata

$$
\ldots \longrightarrow H_{n}(A \cap B) \stackrel{i_{*}}{\rightarrow} H_{n}(A) \oplus H_{n}(B) \stackrel{s_{*}}{\rightarrow} H_{n}(X) \stackrel{\partial_{*}}{\rightarrow} H_{n-1}(A \cap B) \stackrel{i_{*}}{\rightarrow} \ldots
$$

\subsubsection{Bom Par}

Qual a relação entre $H_{n}(X, A)$ e $H_{n}(X / A)$ ?

Definição 2.4.17 $(X, A)$ é um bom par se existir vizinhança aberta $A \subset U \subset X$ tal que $A$ é retrato por deformação de $U$.

Exemplo 2.4.3 (1) Se $N \subset M$ é subvariedade mergulhada, então $(M, N)$ é um bom par.

(2) Se $L \subset K$ é subcomplexo, então $(|K|,|L|)$ é um bom par.

(3) Em particular, se $(K, \partial K)$ é pseudo-variedade com bordo $\partial K \neq \emptyset$, então $(|K|,|\partial K|)$.

(4) O mesmo para $L \subset K$ sub-pseudo-variedade, então $(|K|,|L|)$ é um bom par.

Teorema 2.4.4 Se $(X, A)$ é um bom par, então

$$
H_{n}(X, A) \cong \tilde{H}_{n}(X / A), \forall n
$$

Demonstração 2.4.4.1 Notemos que:

(1) $A / A=\left\{x_{0}\right\}$ é retrato por deformação de $U / A$.

(2) $\pi:(X-A, U-A) \rightarrow(X / A-A / A, U / A-A / A)$ é homeomorfismo e portanto $\pi_{*}$ é isomorfismo. $\operatorname{Logo}$

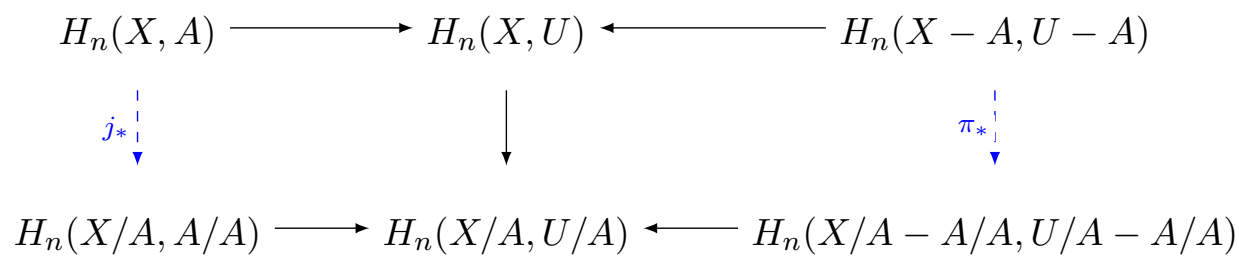

Portanto, $j_{*}$ é isomorfismo. 
Observação 2.4.3 Não é verdade em geral que $H_{n}(X, A) \cong \tilde{H}_{n}(X / A)$.

Teorema 2.4.5 (Invariância de Domínio) Se $U \subset \mathbb{R}^{m}, V \subset \mathbb{R}^{n}$ são abertos e homeomorfos, então $m=n$.

Demonstração 2.4.5.1 Seja $\alpha \in U$, então

$$
H_{k}(U, U-\{x\}) \cong \tilde{H}_{k}\left(\mathbb{R}^{m}, \mathbb{R}^{m}-\{x\}\right)
$$

Por excisão (tomando $B=U, A=\mathbb{R}^{m}-\{x\} \subseteq \mathbb{R}^{m}$ ), da sequência longa do par, vem que:

$$
H_{k}\left(\mathbb{R}^{m}, \mathbb{R}^{m}-\{x\}\right) \cong \tilde{H}_{k}\left(\mathbb{R}^{m}-\{x\}\right) \cong\left\{\begin{array}{ccc}
R & \text { se } & k=m-1 \\
0 & \text { se } & k \neq m-1
\end{array}\right.
$$

Logo, se $\varphi:(U, U-\{x\}) \rightarrow(V, V-\{\varphi(x)\})$ é homeomorfismo, e portanto,

$$
\varphi_{*}: H_{k}(U, U-\{x\}) \rightarrow(V, V-\{\varphi(x)\}) \Longrightarrow m=n .
$$

\subsection{Isomorfismo entre Homologias Simplicial e Singular}

\subsubsection{Homologia Simplicial vs. Homologia Singular}

Seja $K$ um complexo simplicial (ou $\Delta$-Complexo) e $|K|$ sua realização geométrica. Queremos mostrar que:

$$
H_{n}^{\operatorname{Simp}}(K) \cong H_{n}^{\operatorname{Sing}}(|K|), \forall n
$$

Observação 2.5.1 A demonstração que seguirá é análoga ao caso das estruturas de $\Delta$-complexo e portanto obteremos como corolário que

$$
H_{n}^{\Delta}(K) \cong H_{n}^{\operatorname{Simp}}(K) .
$$

Seja $\left(v_{0}, \ldots, v_{p}\right)$ um $p$-simplexo de $K$ segue que $\left(v_{0}, \ldots, v_{p}\right)$ determina $\sigma: \Delta^{p} \rightarrow|K|$ obtemos assim:

$$
\begin{gathered}
\psi: C_{p}^{\operatorname{Simp}}(K) \longrightarrow C_{p}^{\text {Sing }}(|K|) \\
\left(v_{0}, \ldots, v_{p}\right) \longmapsto \sigma
\end{gathered}
$$

Teorema 2.5.1 A aplicação $\psi$ acima definida induz o isomorfismo

$$
\psi_{*}: H_{n}^{\operatorname{Simp}}(K) \longrightarrow H_{n}^{\text {Sing }}(|K|), \forall n .
$$

Devida a importância deste resultado, iremos apresentar a demonstração (lema, processo de indução, etc) nos moldes de Hatcher (2001)

Demonstração 2.5.1.1 Utilizaremos o lema abaixo como resultado auxiliar,

Lema 2.5.1 $H_{n}^{\text {Sing }}\left(\Delta^{n}, \partial \Delta^{n}\right) \cong \mathbb{Z}$, gerado por $\mathbb{1}=i_{n}: \Delta^{n} \longrightarrow \Delta^{n}$

Demonstração 2.5.1.1 (Indução em n:) Para $n=0$, temos $\Delta^{0}=\left\{x_{0}\right\}$, e portanto, $\partial \Delta^{0}=\emptyset$, suponha que $H_{n-1}\left(\Delta_{n-1}, \partial \Delta_{n-1}\right)$ é gerado por $i_{n-1}: \Delta^{n-1} \rightarrow \Delta_{n-1}$.

Seja $\Lambda=\partial \Delta^{n}-\left[e_{0}, \ldots, e_{n-1}\right] \subset \Delta^{n}$, temos a sequência longa exata da tripla $\left(\Delta^{n}, \partial \Delta^{n}, \Lambda\right)$

$$
H_{n}\left(\Delta^{n}, \Lambda\right) \rightarrow H_{n}\left(\Delta^{n}, \partial \Delta^{n}\right) \stackrel{\partial_{*}}{\longrightarrow} H_{n-1}\left(\partial \Delta^{n}, \Lambda\right) \longrightarrow H_{n-1}\left(\Delta^{n}, \Lambda\right)
$$


Mas $\Lambda$ é retrato por deformação de $\Delta^{n}$ e portanto,

$$
H_{n}\left(\Delta^{n}, \Lambda\right) \cong H_{n}(\Lambda, \Lambda)=0
$$

Logo, $\partial_{*}$ é isomorfismo. Note que,

$$
\partial_{*}\left(\llbracket i_{n} \rrbracket\right)=\llbracket \partial_{i_{n}} \rrbracket=\llbracket \sum(-1)^{p} i_{n-1}^{p} \rrbracket,
$$

Onde $i_{n-1}^{p}$ é a identificação canônica de $\Delta^{n-1}$ com a p-ésima face $\left[e_{0}, \ldots, \hat{e}_{p}, \ldots, e_{n}\right]$ de $\Delta^{n}$.

Por outro lado, a inclusão

$$
\begin{aligned}
\Delta_{n-1} & \longrightarrow \partial \Delta^{n} \\
{\left[e_{0}, \ldots, e_{n-1}\right] } & \longmapsto\left[e_{0}, \ldots, e_{n-1}\right]
\end{aligned}
$$

Induz um homeomorfismo

$$
\Delta^{n-1} / \partial \Delta^{n-1} \cong \partial \Delta^{n} / \Lambda
$$

$\log$,

$$
\begin{gathered}
H_{n}\left(\Delta^{n}, \partial \Delta^{n}\right) \stackrel{\partial_{*}}{\longrightarrow} H_{n-1}\left(\partial \Delta^{n}, \Lambda\right) \longrightarrow H_{n-1}\left(\Delta^{n-1}, \partial \Delta^{n-1}\right) \\
\llbracket i_{n} \rrbracket \longmapsto \llbracket \sum \pm i_{n-1}^{p} \rrbracket \longrightarrow \llbracket \pm i_{n-1} \rrbracket
\end{gathered}
$$

E portanto, $\left[i_{n}\right]$ gera $H_{n}\left(\Delta^{n}, \partial \Delta^{n}\right) \cong \mathbb{Z}$.

Demonstração 2.5.1.2 (do teorema) Vamos calcular $H_{n}\left(\left|K^{p}\right|,\left|K^{p-1}\right|\right)$, podemos identificar

$$
\left|K^{p}\right|=\coprod_{\alpha} \Delta_{\alpha}^{p} / \sim
$$

Logo, temos,

$$
\psi: \coprod_{\alpha}\left(\Delta_{\alpha}^{p}, \partial \Delta_{\alpha}^{p}\right) \longrightarrow\left(\left|K^{p}\right|,\left|K^{p-1}\right|\right)
$$

que induz um homeomorfismo

$$
\left(\coprod_{\alpha} \Delta_{\alpha}^{p} / \coprod_{\alpha} \partial \Delta_{\alpha}^{p}\right) \longrightarrow\left|K^{p}\right| /\left|K^{p-1}\right|
$$

E portanto,

$$
\begin{gathered}
H_{n}\left(\left|K^{p}\right|,\left|K^{p-1}\right|\right) \cong H_{n}\left(\coprod_{\alpha} \Delta_{\alpha}^{p}, \coprod_{\alpha} \partial \Delta_{\alpha}^{p}\right)=\left\{\begin{array}{cc}
0 & \text { se } n \neq p \\
\text { livre gerado por } i_{n}^{\alpha} & \text { se } n=p
\end{array}\right. \\
\cong H_{n}^{\operatorname{Simp}}\left(K^{p}, K^{p-1}\right) .
\end{gathered}
$$

\subsection{Aplicações}

Nesta seção apresentaremos o cálculo da homologia da esfera $\mathbb{S}^{2}$. Escolha deste espaço topológico foi feita de modo a enriquecer o estudo do artigo do professor Elon Lages Lima, (Lima (1985a)) que versa sobre a demonstração do teorema de Euler para poliedros.

Apresentaremos formas de resolução do problema com as técnicas apresentas até aqui. 


\subsubsection{Homologia de $\mathbb{S}^{2}$ - via homologia simplicial:}

Para obtermos a homologia de $\mathbb{S}^{2}$, consideramos um 3-simplexo $\left[v_{0}, v_{1}, v_{2}, v_{3}\right]$, e tomamos o bordo deste simplexo $\left(\partial \Delta_{3}\right)$, como $\left|\Delta_{3}\right| \simeq \mathbb{S}^{2}$, escolhemos este último como triangulação para $\mathbb{S}^{2}$.

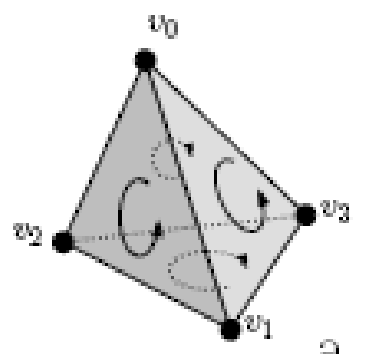

Figura 2.19: Triângulação da Esfera $\mathbb{S}^{2}$

Primeiramente, temos:

$$
\partial_{2}\left(\left[v_{0}, v_{1}, v_{2}, v_{3}\right]\right)=\left[v_{1}, v_{2}, v_{3}\right]-\left[v_{0}, v_{2}, v_{3}\right]+\left[v_{0}, v_{1}, v_{3}\right]-\left[v_{0}, v_{1}, v_{2}\right]
$$

Então, teremos os 2-simplexos a seguir:

$$
A_{1}=\left[v_{1}, v_{2}, v_{3}\right] \quad A_{2}=\left[v_{0}, v_{2}, v_{3}\right] \quad A_{3}=\left[v_{0}, v_{1}, v_{3}\right] \quad A_{4}=\left[v_{0}, v_{1}, v_{2}\right]
$$

Calculando $\partial_{2}\left(A_{i}\right)$, teremos,

$$
\begin{aligned}
\partial_{2}\left(A_{1}\right) & =\partial_{2}\left(\left[v_{1}, v_{2}, v_{3}\right]\right) \\
& =\left[v_{2}, v_{3}\right]-\left[v_{1}, v_{3}\right]+\left[v_{1}, v_{2}\right] \\
\partial_{2}\left(A_{2}\right) & =\partial_{2}\left(\left[v_{0}, v_{2}, v_{3}\right]\right) \\
& =\left[v_{2}, v_{3}\right]-\left[v_{0}, v_{3}\right]+\left[v_{0}, v_{2}\right] \\
\partial_{2}\left(A_{3}\right) & =\partial_{2}\left(\left[v_{0}, v_{1}, v_{3}\right]\right) \\
& =\left[v_{1}, v_{3}\right]-\left[v_{0}, v_{3}\right]+\left[v_{0}, v_{1}\right] \\
& \\
\partial_{2}\left(A_{4}\right) & =\partial_{2}\left(\left[v_{0}, v_{1}, v_{2}\right]\right) \\
& =\left[v_{1}, v_{2}\right]-\left[v_{0}, v_{2}\right]+\left[v_{0}, v_{1}\right]
\end{aligned}
$$

Agora podemos considerar os 1-simplexos $a_{i}$ :

$$
\begin{array}{lll}
a_{1}=\left[v_{0}, v_{1}\right] & a_{2}=\left[v_{1}, v_{2}\right] & a_{3}=\left[v_{0}, v_{2}\right] \\
a_{4}=\left[v_{1}, v_{3}\right] & a_{5}=\left[v_{0}, v_{3}\right] & a_{6}=\left[v_{2}, v_{3}\right]
\end{array}
$$

Podemos calcular para estes $\partial_{1}\left(a_{i}\right)$ :

$$
\begin{array}{ll}
\partial_{1}\left(a_{1}\right)=\partial_{1}\left(\left[v_{0}, v_{1}\right]\right)=v_{1}-v_{0}, & \partial_{1}\left(a_{4}\right)=\partial_{1}\left(\left[v_{1}, v_{3}\right]\right)=v_{3}-v_{1} \\
\partial_{1}\left(a_{2}\right)=\partial_{1}\left(\left[v_{1}, v_{2}\right]\right)=v_{2}-v_{1} & \partial_{1}\left(a_{5}\right)=\partial_{1}\left(\left[v_{0}, v_{3}\right]\right)=v_{3}-v_{0} \\
\partial_{1}\left(a_{3}\right)=\partial_{1}\left(\left[v_{0}, v_{2}\right]\right)=v_{2}-v_{0} & \partial_{1}\left(a_{6}\right)=\partial_{1}\left(\left[v_{2}, v_{3}\right]\right)=v_{3}-v_{2}
\end{array}
$$


Então teremos o complexo de cadeias simplicias considerando estes simplexos:

$$
0 \stackrel{\partial_{3}}{\longrightarrow} C_{2}\left(\mathbb{S}^{2}, \mathbb{Z}\right) \stackrel{\partial_{2}}{\longrightarrow} C_{1}\left(\mathbb{S}^{2}, \mathbb{Z}\right) \stackrel{\partial_{1}}{\longrightarrow} C_{0}\left(\mathbb{S}^{2}, \mathbb{Z}\right) \stackrel{\partial_{0}}{\longrightarrow} 0
$$

Logo,

$$
0 \stackrel{\partial_{3}}{\longrightarrow}\left\langle A_{1}, A_{2}, A_{3}, A_{4}\right\rangle \stackrel{\partial_{2}}{\longrightarrow}\left\langle a_{1}, a_{2}, a_{3}, a_{4}, a_{5}, a_{6}\right\rangle \stackrel{\partial_{1}}{\longrightarrow}\left\langle v_{0}, v_{1}, v_{2}, v_{3}\right\rangle \stackrel{\partial_{0}}{\longrightarrow} 0
$$

E equivalentemente,

$$
0 \stackrel{\partial_{3}}{\longrightarrow} \mathbb{Z}^{4} \stackrel{\partial_{2}}{\longrightarrow} \mathbb{Z}^{6} \stackrel{\partial_{1}}{\longrightarrow} \mathbb{Z}^{4} \stackrel{\partial_{0}}{\longrightarrow} 0 .
$$

Vamos calcular os grupos de homologia de $\mathbb{S}^{2}$ :

Para $H_{0}\left(\mathbb{S}^{2}\right)$, teremos pela proposição 2.2.1 que este complexo simplicial, por ser conexo (apresenta apenas uma componente conexa) esse valor é igual a $\mathbb{Z}$, portanto,

$$
H_{0}\left(\mathbb{S}^{2}, \mathbb{Z}\right)=\frac{\operatorname{Ker}\left(\partial_{0}\right)}{\operatorname{Im}\left(\partial_{1}\right)}=\mathbb{Z}
$$

por analogia aos exemplos iniciais devidos à Gallier e Quaintance (2016) Teremos que para o cálculo de $H_{k}\left(\mathbb{S}^{2}\right), k>0$

$$
H_{k}\left(\mathbb{S}^{2}, \mathbb{Z}\right)=\frac{\operatorname{Ker}\left(\partial_{k}\right)}{\operatorname{Im}\left(\partial_{k+1}\right)}=0
$$

\subsubsection{Homologia de $\mathbb{S}^{2}$ - via Bom Par}

Pela definição 2.4.17 seja $(X, A)=\left(\mathbb{D}^{n}, \mathbb{S}^{n-1}\right)$ um bom par, pela sequência longa do par teremos,

$$
\ldots \rightarrow \tilde{H}_{k}\left(\mathbb{S}^{n-1}\right) \rightarrow \tilde{H}_{k}\left(\mathbb{D}^{n}\right) \rightarrow \tilde{H}_{k}\left(\mathbb{S}^{n}\right) \stackrel{\partial_{*}}{\rightarrow} \tilde{H}_{k-1}\left(\mathbb{S}^{n-1}\right) \rightarrow \tilde{H}_{k-1}\left(\mathbb{D}^{n}\right) \rightarrow \tilde{H}_{k-1}\left(\mathbb{S}^{n}\right) \rightarrow \ldots \rightarrow \tilde{H}_{0}\left(\mathbb{S}^{n-1}\right) \rightarrow 0
$$

Nesta sequência os termos $\tilde{H}_{i}\left(\mathbb{D}^{n}\right)=0, \forall i=0, \ldots, k$, uma vez que $\mathbb{D}^{n}$ é contrátil. e assim

$$
\ldots \rightarrow \tilde{H}_{k}\left(\mathbb{S}^{n-1}\right) \rightarrow 0 \rightarrow \tilde{H}_{k}\left(\mathbb{S}^{n}\right) \stackrel{\partial_{*}}{\rightarrow} \tilde{H}_{k-1}\left(\mathbb{S}^{n-1}\right) \rightarrow 0 \rightarrow \tilde{H}_{k-1}\left(\mathbb{S}^{n}\right) \rightarrow \ldots \rightarrow \tilde{H}_{0}\left(\mathbb{S}^{n-1}\right) \rightarrow 0
$$

De onde destacamos que,

$$
\ldots \rightarrow 0 \rightarrow \tilde{H}_{k}\left(\mathbb{S}^{n}\right) \stackrel{\partial_{*}}{\rightarrow} \tilde{H}_{k-1}\left(\mathbb{S}^{n-1}\right) \rightarrow 0 \rightarrow \ldots
$$

e desta forma, pela exatidão da sequência, estes dos grupos de homologia são isomorfos (ver teorema 2.4.5) para $k>0$ e que $\tilde{H}_{0}\left(\mathbb{S}^{n}\right)=0$.

Podemos concluir, utilizando o processo de indução em $n$, iniciando em $\mathbb{S}^{0}=\{-1,1\}=\{-1\} \sqcup$ $\{1\}$, teremos portanto

$$
\tilde{H}_{k}\left(\mathbb{S}^{n}\right)=\left\{\begin{array}{l}
\mathbb{Z} \text { para } k=n \\
0 \text { para } k \neq n
\end{array}\right.
$$

Fazendo $n=2$ teremos o resultado procurado.

\subsubsection{Homologia de $\mathbb{S}^{2}$ - via Mayer - Vietoris}

Apresentadas as várias formas de calcular a homologia da esfera $\mathbb{S}^{2}$, mostraremos que sua característica de Euler tem valor igual a 2. 


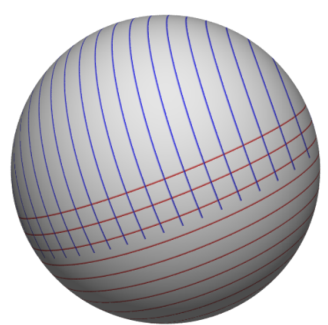

\section{A $A \cap B \quad B$}

Figura 2.20: hipóteses em Mayer - Vietoris

Sejam $A=$ Hemisfério Norte (um pouco abaixo do equador) $\cong D^{2}, B=$ Hemisfério Sul (um pouco acima do equador) $\cong D^{2}$, então $A \cap B \cong S^{1}$ (Homotopicamente Equivalentes) e portanto,

$$
\tilde{H}_{k}(A) \oplus \tilde{H}_{k}(B) \rightarrow \tilde{H}_{k}\left(\mathbb{S}^{2}\right) \rightarrow \tilde{H}_{k-1}\left(\mathbb{S}^{1}\right) \rightarrow \tilde{H}_{k-1}(A) \oplus \tilde{H}_{k-1}(B)
$$

E assim,

$$
\tilde{H}_{k}\left(\mathbb{S}^{2}\right) \cong \tilde{H}_{k-1}\left(\mathbb{S}^{1}\right) \cong \tilde{H}_{k-2}\left(\mathbb{S}^{0}\right)
$$

Mas $\mathbb{S}^{0}=\{-1,1\}=\{-1\} \sqcup\{1\}$ E portanto,

$$
\tilde{H}_{k}\left(\mathbb{S}^{2}\right)=\left\{\begin{array}{lll}
\mathbb{Z} & \text { se } & k=2 \\
0 & \text { se } & k \neq 2
\end{array}\right.
$$

\subsection{4 $\chi\left(\mathbb{S}^{2}\right)=2$}

Pela definição 2.1.16 poderemos calcular a característica de Euler da esfera $\mathbb{S}^{2}$ diretamente pela relação apresentada (figura 2.6.4) segue de $\left|\partial \Delta^{3}\right| \approx\left|\mathbb{S}^{2}\right|$

$$
\begin{aligned}
\chi\left(\mathbb{S}^{2}\right) & =\sum_{i=0}^{3}(-1)^{i} \#\left(\sigma_{i}\right) \\
& =\#\left(\sigma_{0}\right)-\#\left(\sigma_{1}\right)+\#\left(\sigma_{2}\right) \\
& =4-6+4 \\
& =2
\end{aligned}
$$

Esse valor é o mesmo para qualquer triangulação da Esfera $\mathbb{S}^{2}$. 


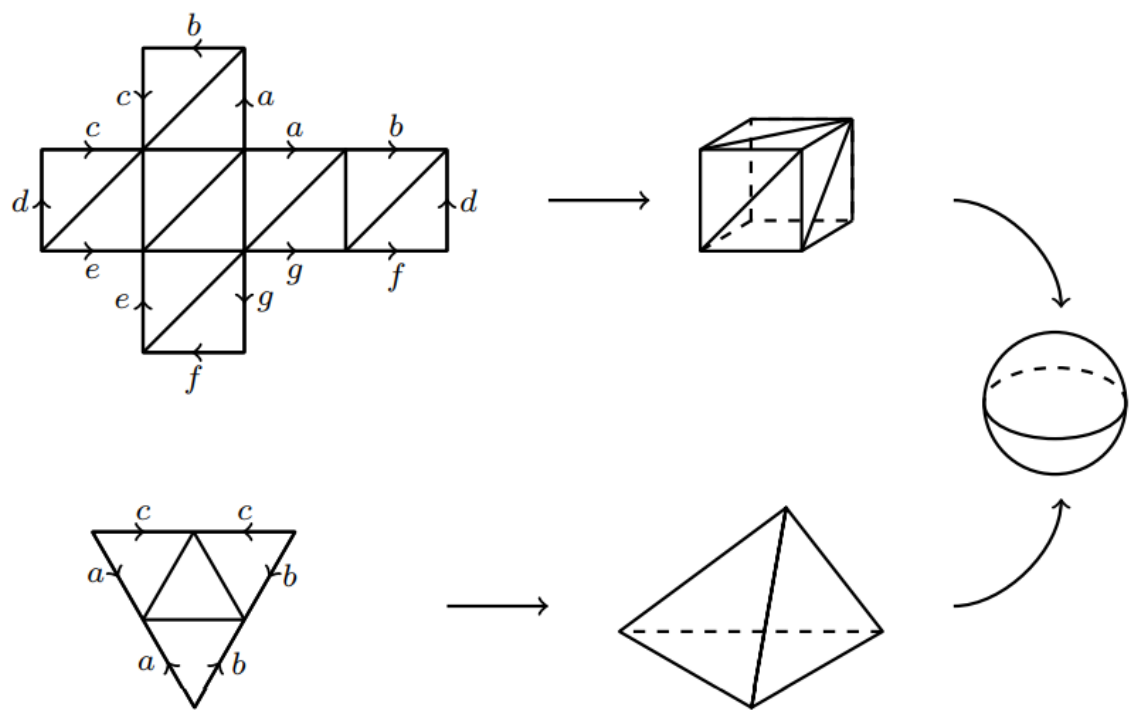

Figura 2.21: Triangulações da Esfera $\mathbb{S}^{2}$.Brasselet e Thuy (2016) 


\section{Capítulo 3}

\section{Teorema de Euler (Cauchy - Lima)}

\subsection{Comentários Iniciais:}

No capítulo anterior foi mostrado, utilizando métodos de homologia, que $\chi(X)=2$ para $X$ homeomorfo a esfera $\mathbb{S}^{2}$. Assim sendo, para qualquer qualquer triangulação $K$ de $\mathbb{S}^{2}$ teremos

$$
\chi(K)=V-A+F=2 .
$$
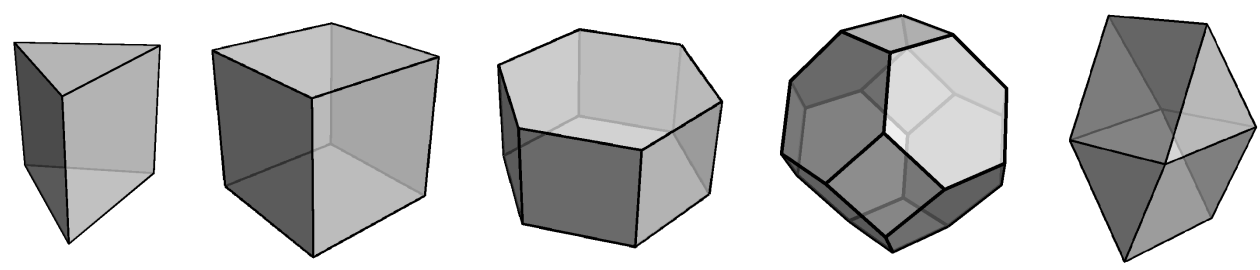

Figura 3.1: Poliedros para os quais o teorema é válido.
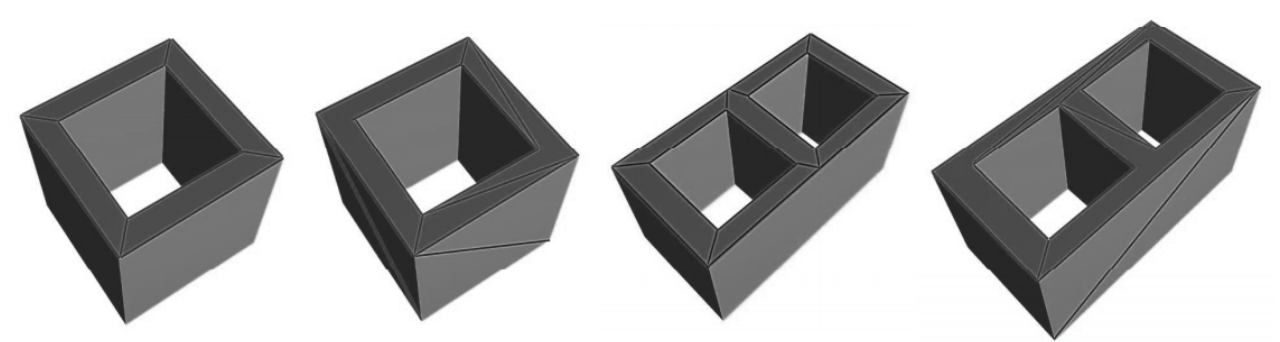

Figura 3.2: Poliedros para os quais o teorema não é válido.

Em Lima (1985a), o autor, motivado pelo texto de Lakatos (1976), explicita o que de fato foi demonstrado por Cauchy na tentativa de verificar o Teorema de Euler e esta demonstração, reformulada em Courant e Robbins (1996) e em Hilbert e Cohn-Vossen (1990), utilizava técnicas avançadas que não foram mencionadas pelos autores (Lima (1985a)) mas são de extrema importância para a validade do resultado.

No caso de poliedros convexos, existem várias demonstrações corretas que utilizam resultados elementares de geometria, o autor destaca a primeira dessas a de Legendre (1849) que foi melhorada em Filho (1983) na qual foi tirada a dependência da geometria esférica (ver Lima (1985b)).

\subsubsection{Teorema de Euler para Poliedros (Cauchy - 1813)}

O enunciado do teorema a ser demonstrado (versão de Cauchy) será reformulado em linguagem moderna utilizando o conceito de pseudo-variedades (definição 2.3.1), o que nos permitirá a conexão 
das demonstrações topológicas com esta, que é geométrica Lima (1984), Lima (1985a), Lima (1985b), Brasselet e Thuy (2016).

O teorema que vamos provar é sobre pseudo-variedades $K$ de dimensão 2 onde todo 1- ciclo é um bordo, ou seja, $H_{1}(K)=0$ e desta forma, $K$ é orientável, pelo teorema 2.3.1 do capítulo 2 .

Exemplo 3.1.1 No toro $\mathbb{T}^{2}$ existe um ciclo que não é bordo!

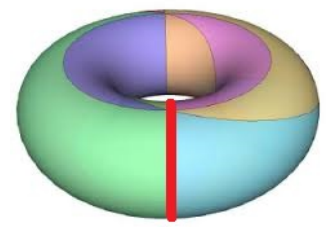

Figura 3.3: Ciclo que não é bordo em $\mathbb{T}^{2}$

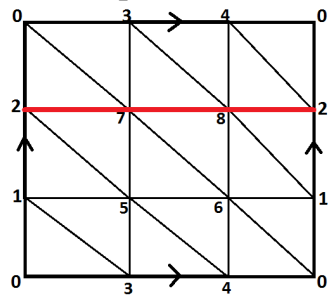

Teorema 3.1.1 Seja $K$, pseudo-variedade, irredutivel, fechada de dimensão 2 , tal que $H_{1}(K)=0$, então:

$$
\chi(K)=V-A+F=2 .
$$

Onde $V:=\#$ 0-simplexos, $A:=\# 1$-simplexos, $F:=\# 2$-simplexos em $K$.

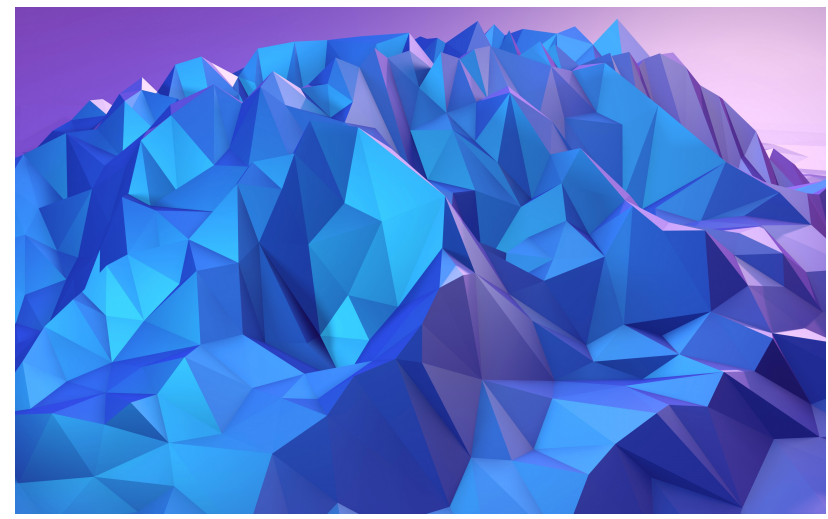

Figura 3.4: Pseudo-variedade de dimensão 2

Observação 3.1.1 A reformulação do enunciado nos remete ao de $K$ homeomorfa a esfera $\mathbb{S}^{2}$ onde $H_{1}\left(\mathbb{S}^{2}\right)=0$, desta forma resultado do teorema é válido e a demonstração já foi apresentada anteriormente. (Ver seção 2.6)

Pelo teorema de Classificação de Superfícies, temos que uma superfície topológica $\Sigma$ compacta e conexa é homeomorfa a esfera $\mathbb{S}^{2}$ se, e somente se, todo ciclo em $\Sigma$ é um bordo. (A única superfície $\Sigma$ fechada tal que $H_{1}(\Sigma)=0$ é a esfera $\mathbb{S}^{2}$, isto é, $H_{1}(\Sigma)=0 \Longleftrightarrow \Sigma=\mathbb{S}^{2}$ ).

Feitas estas considerações, a demonstração de Cauchy ao Teorema de Euler não pode ser feita sem o auxílio de hipóteses da topologia ${ }^{1}$. Seguimos com a demonstração.

\footnotetext{
${ }^{1}$ http://wallpapers4u.net/wallpaper/geometry-4k-734963
} 
Demonstração 3.1.1.1 Sejam $\sigma_{1}, \ldots, \sigma_{k}$ os 2-simplexos de $K$ e $K^{\prime}=K-\left\{\sigma_{k}\right\}$. Notamos que $\chi\left(K^{\prime}\right)=\chi(K)-1$ e além disso, $K$ é conexo com $H_{1}(K)=0$. Devemos observar que a retirada de um 2-simplexo mantém a propriedade de $K$ em $K^{\prime}$ em que todo 1-ciclo é um bordo neste complexo simplicial e isso é, de fundamental importância ao resultado final e portanto enunciaremos esta propriedade.

Proposição 3.1.1 Seja $K^{\prime}$ o complexo simplicial obtido pelo processo de retirada de um 2-simplexo $\sigma_{k}$ de $K$. Então $H_{1}\left(K^{\prime}\right)=0$ onde $K$ está nas condições do teorema 3.1.1.

Demonstração 3.1.1.1 Seja $\gamma \subset K^{\prime}$ um 1-ciclo, pela definição de $K$, existem 2 -simplexos, $\sigma_{i_{1}}, \ldots, \sigma_{i_{p}} \subset$ $K$ tais que,

$$
\partial\left(\sigma_{i_{1}}+\ldots+\sigma_{i_{p}}\right)=\gamma .
$$

Se $i_{l} \neq k, \forall l=1, \ldots, p$ segue o resultado pois, desta forma, $\sigma_{i_{1}}, \ldots, \sigma_{i_{p}}$ serão 2 -simplexos de $K^{\prime}$ e portanto $\gamma$ será bordo em $K^{\prime}$. Caso contrário, sejam $\sigma_{j_{1}}, \ldots, \sigma_{j_{k-p}}$ os outros 2 -simplexos de $K$. Afirmamos que

$$
\partial\left(\sigma_{j_{1}}+\ldots+\sigma_{j_{k-p}}\right)=-\gamma
$$

Pois

$$
\partial\left(\sigma_{i_{1}}+\ldots+\sigma_{i_{p}}+\sigma_{j_{1}}+\ldots+\sigma_{j_{k-p}}\right)=0
$$

E portanto,

$$
{\underline{\partial\left(\sigma_{i_{1}} \pm \ldots+\sigma_{i_{p}}\right)}}^{\gamma}+\partial\left(\sigma_{j_{1}}+\ldots+\sigma_{j_{k-p}}\right)=0
$$

Logo, $\partial\left(\sigma_{j_{1}}+\ldots+\sigma_{j_{k-p}}\right)=-\gamma$, portanto

$$
\sigma_{j_{1}}, \ldots, \sigma_{j_{k-p}} \subset K^{\prime}
$$

E assim, todo 1-ciclo em $K^{\prime}$ é bordo nesse complexo simplicial.

Garantido o resultado anterior passamos agora a retirar, um a um, os 2-simplexos de $K^{\prime}$ que tenham ao menos uma aresta livre e, neste processo, ao retirarmos o 2-simplexo, retiramos junto as arestas e vértices livres. Neste processo vamos estudar como se altera o número de Euler do complexo simplicial restante, bem como o número de componentes conexas.
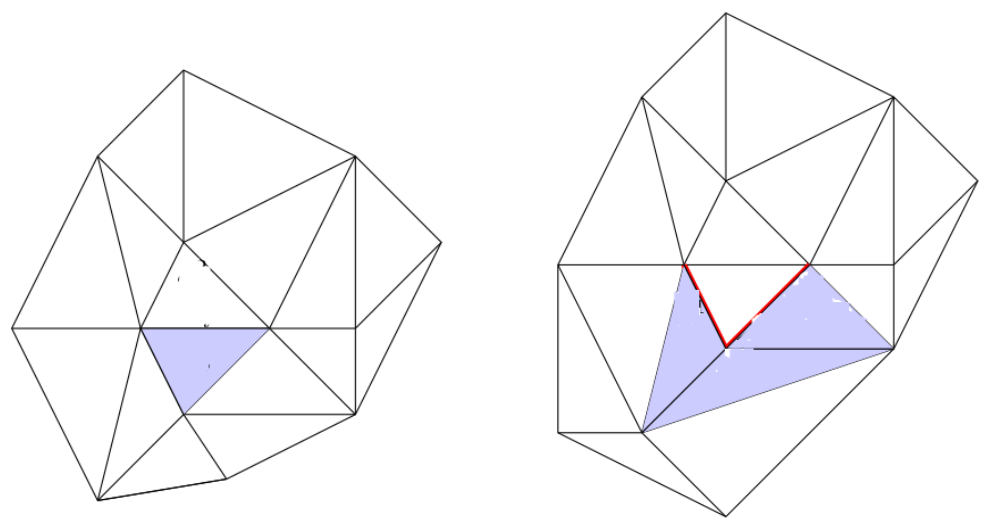

Figura 3.5: Retirada de um 2-simplexo $\sigma_{k}$ da pseudo-variedade $K$. (Brasselet e Thuy (2016))

Observamos que ao longo do processo de retirada dos 2-simplexos existirão 6 possibilidades de escolha para o 2-simplexo a ser retirado, listamos estas possibilidades nas quais o simplexo pode ter: (a) apenas uma aresta livre, (b) 2 arestas livres e 1 vértice livre, (c) 2 arestas livres mas nenhum vértice livre, $(d) 3$ arestas livres e nenhum vértice livre, $(e) 3$ arestas livres e um vértice livre $(f)$ 3 arestas livres e dois vértices livres. Antes de analisar o resultado na característica de Euler $e$ 
no número de componentes conexas que ocorrerá quando retirarmos um 2-simplexo junto com suas arestas e vértices livres, é importante notar que ao longo de todo o processo ainda teremos que todo 1-ciclo é um bordo.

Lema 3.1.1 Sejam L um complexo simplicial que contém um 2-simplexo $\sigma_{0}$ com aresta livre para o qual se tem $H_{1}(L)=0$ e $L^{\prime}$ o complexo simplicial obtido de $L$ retirando $\sigma_{0}$ junto com as arestas e vértices livres deste 2-simplexo, então $H_{1}\left(L^{\prime}\right)=0$.

Demonstração 3.1.1.1 Seja $\gamma \subset L^{\prime}$ um ciclo. Por hipótese, existem em L, 2-simplexos $\sigma_{i_{1}}, \ldots, \sigma_{i_{p}}$ tais que

$$
\partial\left(\sigma_{i_{1}}+\ldots+\sigma_{i_{p}}\right)=\gamma
$$

Afirmamos que $\sigma_{0} \neq \sigma_{i_{j}}, \forall j$, de fato, se

$$
Q=\sigma_{0}+\sigma_{i_{1}}+\ldots+\sigma_{i_{p}}
$$

Então,

$$
\begin{gathered}
\partial(Q)=\partial\left(\sigma_{0}\right)+\partial\left(\sigma_{i_{1}}+\ldots+\sigma_{i_{p}}\right) \\
\partial(Q)=\tau_{\text {livre }}+\text { "outros 1-simplexos" }
\end{gathered}
$$

Absurdo, uma vez que $\gamma \subset L^{\prime}$ que não contém arestas livres $\tau_{\text {livre }}$

Com estas considerações iremos retirar os 2-simplexos até que o complexo simplicial restante seja união disjunta de triângulos (2-simplexos com suas arestas e vértices) Continuamos o processo de retirada dos 2-simplexos de acordo com os casos tipos $(a)$ a $(f)$, um a um, até que cada componente conexa restante tenha somente um único 2 -simplexo e suas faces.

Tipo (a) O 2-simplexo a ser retirado possui uma aresta livre:

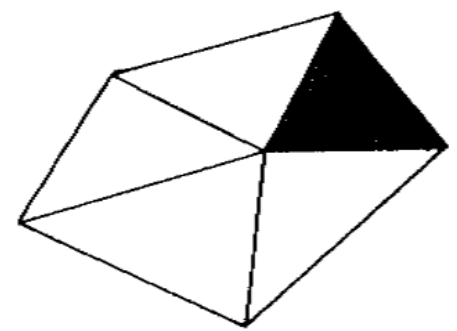

Figura 3.6: $\tau_{1}$ é aresta livre. Lima (1985a)

Neste caso, não teremos a alteração do número de componentes conexas de $K^{\prime}$, mas o número das faces e das arestas diminuirão cada, em uma unidade não alterando assim $\chi\left(K^{\prime}\right)$ inicial. Analisemos agora, outra possibilidade:

Tipo (b) O 2-simplexo a ser retirado possui duas arestas livres e um vértice livre:

$A$ retirada deste 2-simplexo não altera o número de componentes conexas de $K^{\prime}$, mas reduz o número arestas em duas unidades e o de vértices em uma, matendo assim, inalterada a característica de Euler de $K^{\prime}$. Passamos ao caso $(c)$. 


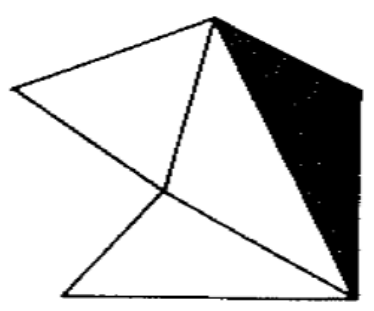

Figura 3.7: $\tau_{1}$ e $\tau_{2}$ são arestas livres. Lima (1985a)

Tipo (c) O 2-simplexo a ser retirado possui duas arestas livres, mas nenhum vértice livre.:

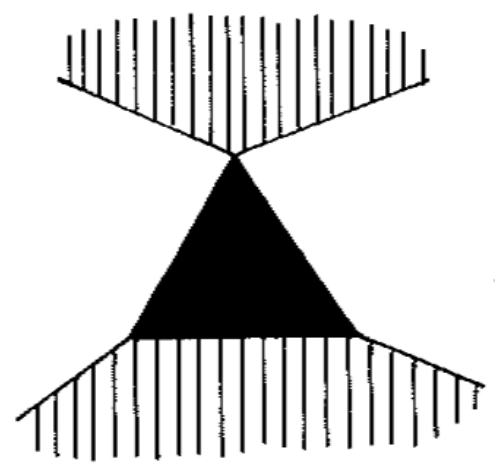

Figura 3.8: $\tau_{1}$ e $\tau_{2}$ são arestas livres, mas nenhum $v$ o é. Lima (1985a)

Neste caso, a retirada do 2-simplexo terá influência direta na conexidade de $K^{\prime \prime}$ frente a $K^{\prime}$ e na determinação de $\chi\left(K^{\prime \prime}\right)$, como veremos a seguir:

Proposição 3.1.2 Seja $\sigma_{l}=(x, y, z)$ o 2-simplexo de que trata o caso $(c)$ onde $\tau_{1}=(x, z)$ $e \tau_{2}=(y, z)$ são as arestas livres. Se $K^{\prime \prime}$ é o complexo simplicial obtido com a retirada de $\sigma_{l}$ (junto com suas arestas livres) então $K^{\prime \prime}$ possui uma componente conexa a mais do que $K^{\prime}$.

Demonstração 3.1.2.1 Devemos mostrar que $x$ e $z$ não podem ser ligados por um caminho de arestas em $K^{\prime \prime}$. Supondo, por absurdo, que exista um caminho $\gamma \subset K^{\prime \prime}$ de arestas ligando $x$ a z. Desta forma, $\gamma \cup \tau_{1}$ é um ciclo em $K^{\prime}$ e como existe uma coleção $Q \subset K^{\prime}$ de 2-simplexos tal que $\partial(Q)=\gamma$, existe outro $\sigma \in Q,\left(\sigma \neq \sigma_{l}\right)$ tal que $\sigma \cap \tau_{1}=\tau_{1}$, isto é, $\tau_{1}$ seria, também, face de $\sigma$, contrariando a hipótese de esta ser livre. Desta forma, $K^{\prime \prime}$ é desconexa, aumentando as componentes conexas de $K^{\prime}$ em uma unidade, logo

$$
\#\left(K^{\prime \prime}\right)=\#\left(K^{\prime}\right)+1
$$

Onde \# $\left(K^{i}\right)$ é o número de componentes conexas de $K^{i}$.

Como retiramos um 2-simplexo (uma face) e dois 2 -simplexos (suas duas arestas) $\tau_{1}, \tau_{2}$ livres, teremos,

$$
\chi\left(K^{\prime \prime}\right)=\chi\left(K^{\prime}\right)+1 .
$$

Tipo (d) O 2-simplexo a ser retirado possui três arestas livres, mas nenhum vértice livre.

De maneira análoga e pela proposição 3.1.2 anterior, cada vértice deste 2-simplexo pertence a uma, e somente uma componente conexa em $K^{\prime \prime}$. Desta forma o número de componentes 


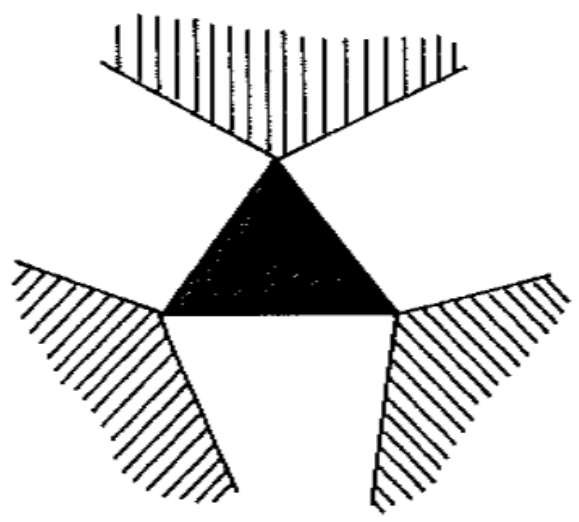

Figura 3.9: $\tau_{1}, \tau_{2}, \tau_{3}$ são arestas livres, com nenhum vértice livre. Lima (1985a)

conexas aumenta em duas unidades.

$$
\#\left(K^{\prime \prime}\right)=\#\left(K^{\prime}\right)+2
$$

Como retiramos o 2-simplexo com suas três arestas livres, diminuímos este número em três e o de faces em uma unidade, portanto,

$$
\chi\left(K^{\prime \prime}\right)=\chi\left(K^{\prime}\right)+2
$$

Tipo (e) O 2-simplexo a ser retirado possui três arestas livres e um vértice livre.

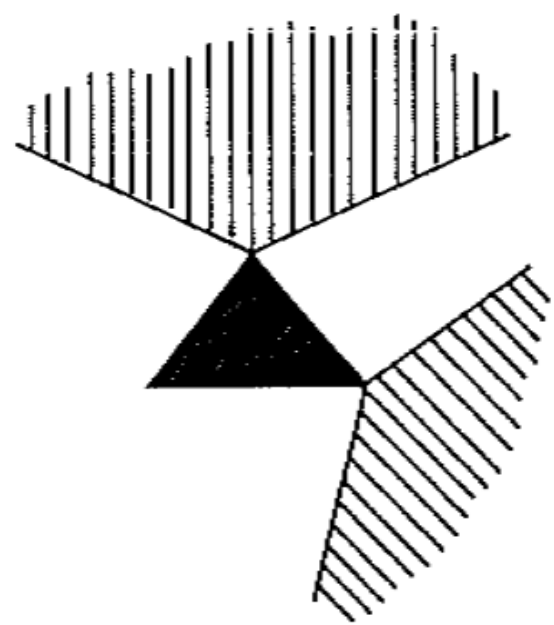

Figura 3.10: $\tau_{1}, \tau_{2}, \tau_{3}$ são arestas livres é $v_{0}$ vértice livre. Lima (1985a)

Teremos novamente dois vértices pertencentes da componentes conexas distintas, então, com a retirada deste 2-simplexo o número de componentes conexas aumenta em uma unidade $e$ portanto,

$$
\#\left(K^{\prime \prime}\right)=\#\left(K^{\prime}\right)+1
$$

Verificamos, agora, que esta retirada altera a característica de Euler de $K^{\prime}$ em uma unidade uma vez que são retiradas três arestas e um vértice livres.

$$
\chi\left(K^{\prime \prime}\right)=\chi\left(K^{\prime}\right)+1
$$

Tipo $(f)$ O 2-simplexo a ser retirado possui três arestas livres e dois vértices livres. 


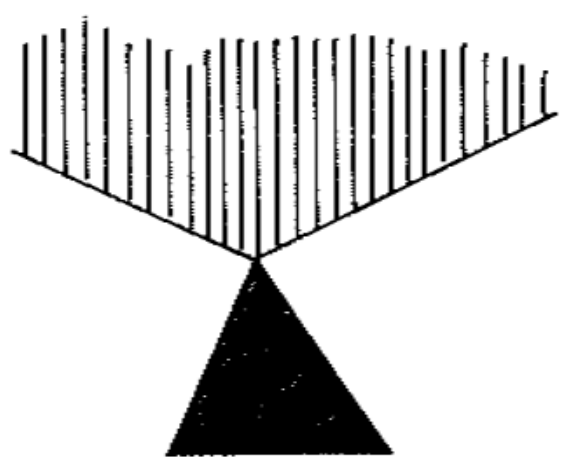

Figura 3.11: $\tau_{1}, \tau_{2}, \tau_{3}$ são arestas livres e $v_{0}, v_{1}$ são vértices livres. Lima (1985a)

Neste caso não teremos alteração no número de componentes conexas de $K^{\prime}$, e a característica de Euler, uma vez que são retiradas três arestas e dois vértices livres, portanto,

$$
\chi\left(K^{\prime \prime}\right)=\chi\left(K^{\prime}\right)
$$

O processo continua de maneira indutiva e como é garantida a propriedade de que todo 1-ciclo é bordo no complexo simplicial seguinte a retirada, estaremos, novamente, frente a algum dos casos (a) até $(f)$ e este processo será finito e bastaremos analisar as componentes conexas e a característica de Euler do complexo formado pela união de todos os subcomplexos simpliciais.

Conclusão 3.1.1 Ao final do processo de retirada, o complexo simplicial final $\left(K_{\text {final }}\right)$ será formado por $(l+1)$ triângulos disjuntos e portanto,

$$
\chi\left(K_{\text {final }}\right)=l+1
$$

Por outro lado, vimos que $\chi\left(K^{\prime}\right)=\chi\left(K_{\text {final }}\right)-l$ pois só aumentamos a característica de Euler quando aumentamos o número de componentes conexas, isto é,

$$
\chi\left(K_{\text {final }}\right)-\chi\left(K^{\prime}\right)=\#\left(K_{\text {final }}\right)-\#\left(K^{\prime}\right)
$$

Além disso, temos que

$$
\chi\left(K^{\prime}\right)=\chi(K)-1
$$

E portanto,

$$
\chi(K)=\chi\left(K^{\prime}\right)+1
$$

Seque que,

$$
\chi(K)=\left[\chi\left(K_{\text {final }}\right)-l\right]+1
$$

E finalmente,

$$
\chi(K)=\chi+1-\not+1=2
$$

\subsection{Conclusão}

A demonstração apresentada neste trabalho com carater geométrico-elementar tem como consequência fundamental o fato de que para espaços homotopicamente equivalentes (hipótese mais fraca de que o homeomorfismo) vale o resultado apresentado, ganhamos assim, uma vasta coleção de exemplares para o ensino deste famoso resultado. 
TEOREMA DE EULER (CAUCHY - LIMA) 


\section{Bibliografia}

Brasselet e Thuy(2016) Jean-Paul Brasselet e Nguyên Thi Bích Thuy. A Caracterítica de EulerPoincaré. https://arxiv.org/pdf/1611.03918.pdf, 2016. Citado na pág. xv, 10, 40, 42, 43

Bredon(1993) Glen E. Bredon. Topogy and Geometry - Graduate texts in Mathematics - vol. 139. Springer - Verlag. Citado na pág. 23

Cauchy(1813) A. L. Cauchy. "Recherche sur les polyèdres - premier mémoire", Journal de l'Ecole Polytechnique 9 (1813), 66-86., 1813. Citado na pág. ix, xi

Courant e Robbins(1996) Richard Courant e Herbert Robbins. What is Mathematics? - An elementary approach to ideas and methods. Oxford University Press. Citado na pág. 41

Filho(1983) Zoroastro Azambuja Filho. Demonstração do Teorema de Euler para poliedros convexos - RPM 03. https://www.ime.usp.br/ pleite/pub/artigos/elon/rpm3.pdf, 1983. Citado na pág. 41

Gallier e Quaintance(2016) Jean Gallier e Jocelyn Quaintance. A Gentle Introduction to Homology - Cohomology and Sheaf Cohomology. http://www.seas.upenn.edu/ jean/ sheaves-cohomology ·pdf, 2016. Citado na pág. xv, 2, 13, 14, 15, 17, 38

Hatcher(2001) Allen Hatcher. Algebraic Topology. Cambridge University Press. Citado na pág. xv, $18,27,34,35$

Hilbert e Cohn-Vossen(1990) David Hilbert e S. Cohn-Vossen. Geometry and Imagination. Chelsea - New York. Citado na pág. 41

Hoffman e Kunze(1971) Kenneth Hoffman e Ray Kunze. Linear Algebra. Prentice-Hall, Inc. Second Edition. Citado na pág. 3

Kinsey(1993) L. Christine Kinsey. Topology of Surfaces. Springer - Verlag. Undergraduate Texts in Mathematics. Citado na pág. xv, 10, 18

Lakatos(1976) Imre Lakatos. Proofs and Refutations - The logic of mathematical discovery. Cambridge University Press. Cambridge Philosophy Classic Edition. Citado na pág. 41

Legendre(1849) A. M. Legendre. Éléments de Géométrie. Librairie de Firmin Didot Frères. Citado na pág. 41

Lima(2012) Elon Lages Lima. Homologia Básica. IMPA, 2 edição. Projeto Euclides. Citado na pág. $4,6,8,12,22,33$

Lima(1984) Elon Lages Lima. Ainda Sobre o Teorema de Euler para Poliedros Convexos- RPM05. http://rpm.org.br/cdrpm/5/6.htm, 1984. Citado na pág. 10, 42

Lima(1985a) Elon Lages Lima. O Teorema de Euler Sobre Poliedros - RPM02. https://rmu . sbm.org.br/wp-content/uploads/sites/27/2018/03/n02_Artigo03.pdf, 1985a. Citado na pág. ix, xi, xv, 1, 2, 36, 41, 42, 44, 45, 46, 47 
Lima(1985b) Elon Lages Lima. A Característica de Euler - Poincaré - RMU01. https://rmu . sbm.org.br/wp-content/uploads/sites/27/2018/03/n01_Artigo02.pdf, 1985b. Citado na pág. $10,41,42$

Massey(1967) William S. Massey. Algebraic Topology, An introduction. Harcourt, Brace World, Inc. Graduate Texts in Mathematics, 56. Citado na pág. 7

Munkres(1984) James R. Munkres. Elements of Algebraic Topology. Addison-Wesley Publishing Company, Inc. Citado na pág. xv, 5, 6, 11

Munkres(1966) James R. Munkres. Elementary Differential Topology. Oxford University Press. Annals of Math. Studies 54. Citado na pág. 7

Prasolov(2006) Viktor Vasil'evich Prasolov. Elements of Combinatorial and Differential Topology. AMS. GSM -74. Citado na pág. 21

Richeson(2012) David S. Richeson. Euler's Gem: The Polyhedron Formula and the Birth of Topology. Princeton University Press. Princeton and Oxford. Citado na pág. 1

Rotman(1988) Joseph J. Rotman. An Introduction to Algebraic Topology. Springer-Verlag. Graduate Texts in Mathematics. Citado na pág. 3 